\title{
Équation anisotrope de Navier-Stokes dans des espaces critiques
}

Marius Paicu

\begin{abstract}
We study the tridimensional Navier-Stokes equation when the value of the vertical viscosity is zero, in a critical space (invariant by the scaling). We shall prove local in time existence of the solution, respectively global in time when the initial data is small compared with the horizontal viscosity.
\end{abstract}

\section{Introduction}

On considère dans la suite le système de Navier-Stokes avec viscosité verticale nulle

$$
\left(N S_{\nu_{\mathrm{v}=0}}\right)\left\{\begin{array}{l}
\partial_{t} v+v \cdot \nabla v-\nu_{h}\left(\partial_{x_{1}}^{2}+\partial_{x_{2}}^{2}\right) v=-\nabla p \quad \text { dans } \mathbb{R}_{+} \times \mathbb{R}^{3} \\
\operatorname{div} v=0 \quad \text { dans } \mathbb{R}_{+} \times \mathbb{R}^{3} \\
\left.v\right|_{t=0}=v_{0}
\end{array}\right.
$$

où $v$ est la vitesse du fluide, $p$ est un scalaire qui représente la pression et $\nu_{h}>0$, est la viscosité horizontale du fluide.

Ce système a été étudié pour la première fois dans [6]. Il provient de l'étude des fluides tournants, dont les équations sont obtenues en ajoutant à l'équation de Navier-Stokes tridimensionnelle, la force de Coriolis $\frac{1}{\varepsilon} v \times e_{3}$, où $e_{3}$ est le vecteur $(0,0,1)$ et $\varepsilon>0$ est le nombre de Rossby, que l'on considère comme un petit paramètre (voir [17]). La loi d'Ekman pour les fluides tournants qui évoluent dans un domaine avec bord nous montre qu'il est pertinent de considérer une viscosité anisotrope, dite "turbulente", de la forme $\left(-\nu_{h} \Delta_{h} v-\beta \varepsilon \partial_{3}^{2} v\right)$ (voir l'article [10]).

2000 Mathematics Subject Classification : 35Q30, 76D03, 76D05.

Keywords : Navier-Stokes equation, critical spaces, anisotropic viscosity. 
Dans [6], pour l'équation de Navier-Stokes sans viscosité dans la direction verticale, est étudiée dans le cas de l'espace entier, l'existence locale en temps pour des données initiales quelconques dans $H^{0, s}\left(\mathbb{R}^{3}\right)\left(s>\frac{1}{2}\right)$ et l'existence globale pour des données petites par rapport à la viscosité horizontale, dans le même espace. L'unicité est démontrée pour $s>\frac{3}{2}$ dans [6] et pour $s>\frac{1}{2}$ dans [13]. Notons que $H^{0, s}\left(\mathbb{R}^{3}\right)$ est l'espace des fonctions qui sont $L^{2}$ dans la variable horizontale et $H^{s}$ dans la variable verticale.

Il est important de penser à la propriété d'invariance de l'équation par changement d'échelle : si $v$ est une solution de l'équation $\left(N S_{\nu_{\mathrm{v}}=0}\right)$ avec donnée initiale $v_{0}$, alors $v_{\lambda}(t, x):=\lambda v\left(\lambda^{2} t, \lambda x\right)$ est encore une solution de l'équation $\left(N S_{\nu_{\mathrm{v}}=0}\right)$, avec donnée initiale $v_{0, \lambda}(x):=\lambda v_{0}(\lambda x)$. Notons que l'espace invariant par ce changement d'échelle est ici $H^{0,1 / 2}$. Une telle approche a été initiée par Fujita et Kato (voir [9]) pour l'équation traditionelle de Navier-Stokes tridimensionelle, dans le cadre de l'espace classique de Sobolev $H^{1 / 2}\left(\mathbb{R}^{3}\right)$.

Le but de cet article est de reprendre les résultats démontrés dans [6] dans un espace invariant par changement d'échelle et "proche" de $H^{0,1 / 2}$. Remarquons que l'absence de viscosité dans une direction empêche d'utiliser une méthode "à la Leray" pour définir des solutions faibles dans l'espace d'énergie $L^{2}\left(\mathbb{R}^{3}\right)$.

Nous allons travailler avec la donnée initiale dans un espace mixte, de type "Besov-Sobolev" anisotrope, qui est un espace critique dans le sens où il est invariant par rapport au changement d'échelle de l'équation de NavierStokes. Cet espace contient les espaces de Sobolev anisotropes $H^{0, s}$ avec $s>1 / 2$. Il est l'espace de régularité minimale pour lequel nous avons pu démontrer l'existence locale et l'unicité pour la solution de l'équation de Navier-Stokes avec viscosité verticale nulle. Ces espaces ont été introduits dans [11] et [12] pour étudier l'existence globale et l'unicité des solutions de l'équation classique de Navier Stokes avec conditions aux limites périodiques, dans le cas où la partie tridimensionelle de la donnée initiale est petite par rapport à la partie bidimensionnelle et à la viscosité.

\section{Notations et énoncés des résultats}

Avant d'énoncer les résultats, définissons les espaces avec lesquels on travaille. On s'intéresse dans cet article à des résultats d'existence et d'unicité pour l'équation $\left(N S_{\nu_{\mathrm{v}=0}}\right)$ dans un espace invariant par changement d'échelle. On peut vérifier facilement que l'espace critique (c'est-à-dire invariant par changement d'échelle) est ici l'espace $H^{0,1 / 2}$. En effet, dans le cas de l'espace entier on remplace l'espace non-homogène $H^{0,1 / 2}$ par sa version homogène 
qu'on introduit ci-dessous

$$
\dot{H}^{0, s}\left(\mathbb{R}^{3}\right)=\left\{\left.u \in \mathcal{S}^{\prime}\left(\mathbb{R}^{3}\right)\left|\|u\|_{\dot{H}^{0, s}}^{2}:=\int_{\mathbb{R}^{3}}\right| \xi_{3}\right|^{2 s}|\hat{u}(\xi)|^{2} d \xi<\infty\right\} .
$$

Rappelons que l'espace non-homogène $H^{0, s}$ est obtenu en remplaçant $\left|\xi_{3}\right|^{2 s}$ par $\left(1+\left|\xi_{3}\right|^{2}\right)^{s}$, dans la définition ci-dessus.

Pour atteindre le cas limite $s=1 / 2$, nous allons remplacer l'espace de Sobolev anisotrope $H^{0, \frac{1}{2}}$ par l'espace mixte "Besov-Sobolev" anisotrope $\mathcal{B}^{0,1 / 2}$. Introduisons cet espace par l'adhérence de fonctions régulières pour la norme:

$$
\|u\|_{\mathcal{B}^{0,1 / 2}}=\sum_{q \in \mathbb{Z}}\left(\int_{\xi_{h}} \int_{2^{q-1}<\left|\xi_{3}\right|<2^{q}}\left|\xi_{3} \| \hat{u}(\xi)\right|^{2} d \xi\right)^{1 / 2}<\infty .
$$

Il est facile de voir que cette norme est équivalente à la norme suivante

$$
\|u\|_{\mathcal{B}^{0,1 / 2}}=\sum_{q \in \mathbb{Z}} 2^{q / 2}\left\|\triangle_{q}^{\mathrm{v}} u\right\|_{L^{2}\left(\mathbb{R}^{3}\right)}<\infty
$$

où $\triangle_{q}^{\mathrm{v}}$ désigne les opérateurs de localisation en fréquences verticales (voir la définition dans la section 3).

Cet espace contient les espaces $H^{0, s}(s>1 / 2)$ et il est un peu plus petit que $H^{0,1 / 2}$ mais il a les mêmes proprietés d'invariance par changement d'échelle. La raison pour laquelle on utilise l'espace $\mathcal{B}^{0,1 / 2}$ au lieu de $H^{0,1 / 2}$ est donnée par le fait que cet espace est inclus dans $L^{\infty}\left(\mathbb{R}_{x_{3}} ; L^{2}\left(\mathbb{R}_{x_{1}} \times \mathbb{R}_{x_{2}}\right)\right)=$ : $L_{\mathrm{v}}^{\infty} L_{h}^{2}$ et l'inclusion de $H^{0, s}$ (avec $s>1 / 2$ ) dans l'espace $L_{\mathrm{v}}^{\infty}\left(L_{h}^{2}\right)$ a joué un rôle important dans les démonstrations données dans [6].

Le théorème principal de l'article est un résultat d'existence locale et d'unicité des solutions pour l'équation de Navier-Stokes avec viscosité verticale nulle. Nous allons démontrer l'existence et l'unicité de la solution dans l'espace $\mathcal{B}^{0,1 / 2}$ (voir le théorème 1 ). Nous démontrons également l'unicité de la solution de l'équation $\left(N S_{\nu_{\mathrm{v}}=0}\right)$ lorsque la donnée initiale est dans l'espace non-homogène $H^{0,1 / 2}$, espace qui n'est pas comparable avec $\mathcal{B}^{0,1 / 2}$ ni avec $C^{-1}$ (voir le théorème 2). Rappelons que la norme de l'espace de Hölder-Zygmund $C^{-1}$ est donnée par $\|u\|_{C^{-1}}=\sup _{q \in \mathbb{Z}} 2^{-q}\left\|\triangle_{q} u\right\|_{L^{\infty}}<+\infty$ (voir la définition 6.1). L'intérêt de cet espace est le fait qu'on connait l'unicité de la solution de l'équation classique de Navier-Stokes tridimensionelle dans $C^{-1}$. Ce résultat a été démontré par J.-Y. Chemin dans [4]. Signalons que l'espace maximal où l'on connait l'existence et l'unicité de la solution pour l'équation de Navier-Stokes tridimensionelle avec viscosités positives dans toutes les directions est l'espace $\partial B M O$ (voir l'article [14]). En outre, dans le théorème 1 , on donne une condition d'explosion en temps fini. 
Théorème 1 Soit $v_{0} \in \mathcal{B}^{0,1 / 2}\left(\mathbb{R}^{3}\right)$ un champ de vecteurs de divergence nulle. Alors, il existe un temps $T>0$ tel que l'équation $\left(N S_{\nu_{\mathrm{v}}=0}\right)$ a une unique solution

$$
v \in C_{b}\left([0, T], \mathcal{B}^{0,1 / 2}\right) \text { avec } \nabla_{h} v \in L^{2}\left([0, T], \mathcal{B}^{0,1 / 2}\right) .
$$

De plus, il existe une constante c telle que si

$$
\left\|v_{0}\right\|_{\mathcal{B}^{0,1 / 2}} \leq c \nu_{h}
$$

alors, l'équation $\left(N S_{\nu_{\mathrm{v}}=0}\right)$ admet une unique solution globale en temps

$$
v \in C_{b}\left(\mathbb{R}_{+}, \mathcal{B}^{0,1 / 2}\right) \text { avec } \nabla_{h} v \in L^{2}\left(\mathbb{R}_{+}, \mathcal{B}^{0,1 / 2}\right) .
$$

Soit $T^{*}$ le temps de vie maximal pour $v$. On a

$$
\text { Si } T^{*}<\infty \text { alors }\|v\|_{\widetilde{L_{T^{*}}^{\infty}}\left(\mathcal{B}^{0,1 / 2}\right)}=+\infty
$$

Le théorème suivant est un résultat d'unicité dans un espace qui n'est pas inclus dans $C^{-1}$. Notons par $H^{1,1 / 2}$ l'espace des fonctions dont le gradient dans la variable horizontale appartient à l'espace $H^{0,1 / 2}$.

Théorème 2 L'équation $\left(N S_{\nu_{\mathrm{v}}=0}\right)$ admet au plus une solution dans l'espace

$$
L_{T}^{\infty}\left(H^{0,1 / 2}\right) \cap L_{T}^{2}\left(H^{1,1 / 2}\right) .
$$

Remarque 2.1 Notons que la démonstration de l'unicité montre également que l'appliquation $S(t)$ qui à la donnée initiale $u_{0} \in \mathcal{B}^{0,1 / 2}$ associe l'unique solution $u(t):=S(t) u_{0}$ de l'équation $\left(N S_{\nu_{\mathrm{v}}=0}\right)$ avec $u \in L_{T}^{\infty}\left(\mathcal{B}^{0,1 / 2}\right)$ et $\nabla_{h} u \in$ $L_{T}^{2}\left(\mathcal{B}^{0,1 / 2}\right)$ est une fonction continue de $\mathcal{B}^{0,1 / 2}$ dans $H^{0,-1 / 2}$. Rappelons que la solution de l'équation classique de Navier-Stokes dépend de façon localement lipschitzienne de la donnée initiale.

Le plan de l'article est le suivant :

- Dans la deuxième section, nous introduisons les fondements de la théorie de Littlewood-Paley, outil de base de tout ce travail.

- Dans la troisième section, nous énonçons les différentes estimations d'énergie nécessaires à la démonstration de l'existence et l'unicité locale (respectivement globale quand la donnée initiale est petite) pour l'équation de Navier-Stokes et nous démontrons les théorèmes 1 et 2 en utilisant ces estimations. Rappelons ici une forme particulière de l'estimation d'énergie obtenue dans [6] :

$$
\left|\left(\triangle_{q}^{\mathrm{v}}(v \cdot \nabla v) \mid \triangle_{q}^{\mathrm{v}} v\right)\right| \leq 2^{-2 q s} C c_{q}\left\|\nabla_{h} v\right\|_{H^{0, s}}^{2}\|v\|_{H^{0, s}} ; \quad \text { où } c_{q} \in \ell_{q}^{2}
$$


On sait que de telles estimations donnent pour l'équation de NavierStokes l'existence globale pour des données petites. Nous allons donner une estimation d'énergie dans le même esprit que celle ci-dessus, mais dans le cadre des espaces de Besov, ce qui permettra d'obtenir l'existence globale pour des données initiales petites.

On peut obtenir aussi l'existence locale pour des données quelconques, en décomposant la donnée initiale en une partie "basses fréquences" (qui comme en général pour les problèmes locaux en temps ne jouera pas un rôle important), et une partie "hautes fréquences", qui sera petite.

L'unicité est plus délicate et demande une estimation doublement logarithmique de la forme

$$
\varphi^{\prime}(t) \leq f(t) \varphi(t)(-\ln \varphi(t)) \ln (-\ln \varphi(t)),
$$

où $\varphi$ est une norme sur la différence entre deux solutions dans un espace de Banach convenable et $f$ est une fonction localement intégrable. Le lemme d'Osgood permet alors de conclure.

- La quatrième section est dédiée aux démonstrations des estimations d'énergie énoncées dans la troisième section. Il s'agit, entre autres, de démontrer l'inégalité suivante :

$$
\int_{0}^{T}\left|\left(\triangle_{q}^{\mathrm{v}}(v \cdot \nabla v)(t) \mid \triangle_{q}^{\mathrm{v}} v(t)\right)_{L^{2}}\right| d t \leq C 2^{-q} a_{q}\left\|\nabla_{h} v\right\|_{\widetilde{L_{T}^{2}}\left(\mathcal{B}^{0,1 / 2}\right)}^{2}\|v\|_{\widetilde{L_{T}^{\infty}}\left(\mathcal{B}^{0,1 / 2}\right)}
$$

avec $\sum_{q} a_{q}^{1 / 2}=1$. Pour la définition des espaces utilisés ici, voir la relation (3.12). Cette inégalité est analogue à l'estimation (2.1).

Pour démontrer l'unicité on démontre l'estimation (2.2) avec $\varphi(t)=$ $\|w(t)\|_{H^{0,-1 / 2}}^{2}$ où $w$ est la différence entre deux solutions de l'équation $\left(N S_{\nu_{\mathrm{v}}=0}\right)$. Remarquons que $w$ est estimée dans $H^{0,-1 / 2}$ bien qu'elle appartienne à l'espace $H^{0,1 / 2}$. Ceci est dû au fait que l'équation satisfaite par la différence entre deux solutions est effectivement hyperbolique dans la variable verticale, ce qui justifie une perte d'une dérivée en variable verticale dans les estimations.

- Dans la dernière section on démontre la non-inclusion de l'espace $\mathcal{B}^{0,1 / 2}$ dans les espaces de Besov isotropes $B_{p, \infty}^{-1+3 / p}\left(\mathbb{R}^{3}\right)$ avec $p \neq \infty$, pour lesquels on connait l'existence locale de la solution pour l'équation de Navier-Stokes classique par les travaux de Cannone et Planchon (voir [2] et [18], [19]). Pour $p=\infty$ nous avons $\mathcal{B}^{0,1 / 2} \subset B_{\infty, \infty}^{-1}=C^{-1}$. Remarquons que l'espace de Sobolev non-homogène $H^{0,1 / 2}\left(\mathbb{R}^{3}\right)$ où on démontre l'unicité pour l'équation $\left(N S_{\nu_{\mathrm{v}}=0}\right)$ n'est pas inclus dans l'espace $C^{-1}$ où on connaissait l'unicité pour l'équation classique de NavierStokes (voir [4]). 


\section{Préliminaires}

Tout d'abord, rappelons la définition des espaces de Lebesgue anisotropes. On désigne par $L_{h}^{p}\left(L_{\mathrm{v}}^{q}\right)$ l'espace $L^{p}\left(\mathbb{R}_{x_{1}} \times \mathbb{R}_{x_{2}} ; L^{q}\left(\mathbb{R}_{x_{3}}\right)\right)$ qui est muni de la norme :

$$
\begin{aligned}
\|f\|_{L_{h}^{p}\left(L_{\mathrm{v}}^{q}\right)} & :=\|\| f\left(x_{h}, \cdot\right)\left\|_{L^{q}\left(\mathbb{R}_{x_{3}}\right)}\right\|_{L^{p}\left(\mathbb{R}_{x_{1}} \times \mathbb{R}_{x_{2}}\right)} \\
& =\left(\int_{\mathbb{R}_{x_{1}} \times \mathbb{R}_{x_{2}}}\left(\int_{\mathbb{R}_{x_{3}}}\left|f\left(x_{h}, x_{3}\right)\right|^{q} d x_{3}\right)^{p / q} d x_{h}\right)^{1 / p} .
\end{aligned}
$$

De la même manière, on note par $L_{\mathrm{v}}^{q}\left(L_{h}^{p}\right)$ l'espace $L^{q}\left(\mathbb{R}_{x_{3}} ; L^{p}\left(\mathbb{R}_{x_{1}} \times \mathbb{R}_{x_{2}}\right)\right)$ avec la norme associée $\|f\|_{L_{\mathrm{V}}^{q}\left(L_{h}^{p}\right)}:=\|\| f\left(\cdot, x_{3}\right)\left\|_{L^{p}\left(\mathbb{R}_{x_{1} \times \mathbb{R}_{x_{2}}}\right)}\right\|_{L^{q}\left(\mathbb{R}_{x_{3}}\right)}$.

C'est bien connu que l'ordre d'intégration est important dans le sens décrit par le lemme ci-après :

Lemme 3.1 Soient $1 \leq p \leq q$ et $f: X_{1} \times X_{2} \rightarrow \mathbb{R}$ une fonction appartenant à $L^{p}\left(X_{1} ; L^{q}\left(X_{2}\right)\right)$, où $\left(X_{1} ; d \mu_{1}\right)$ et $\left(X_{2} ; d \mu_{2}\right)$ sont des espaces mesurables. Alors $f \in L^{q}\left(X_{2} ; L^{p}\left(X_{1}\right)\right)$ et on a l'inégalité

$$
\|f\|_{L^{q}\left(X_{2} ; L^{p}\left(X_{1}\right)\right)} \leq\|f\|_{L^{p}\left(X_{1} ; L^{q}\left(X_{2}\right)\right)} .
$$

L'outil de base dans les démonstrations sera la théorie de Littlewood-Paley anisotrope qui consiste à faire un découpage dyadique dans les fréquences verticales. On va faire ainsi les estimations d'énergie sur chaque bloc dyadique et ensuite on va sommer les estimations obtenues.

Introduisons les opérateurs de troncature en fréquences verticales comme suit:

$$
\triangle_{q}^{\mathrm{v}} u=\mathcal{F}^{-1}\left(\varphi\left(\frac{\left|\xi_{3}\right|}{2^{q}}\right) \mathcal{F} u(\xi)\right)
$$

où on a noté par $\mathcal{F}$ la transformation de Fourier et $u \in \mathcal{S}^{\prime}\left(\mathbb{R}^{3}\right)$.

Les fonctions $\varphi$ et $\chi$ représentent une partition de l'unité dans $\mathbb{R}$, elles sont régulières et on a

$$
\text { supp } \chi \subset B(0,4 / 3), \text { supp } \varphi \subset \mathcal{C}(3 / 4,8 / 3) \text {, }
$$

et

$$
\chi(t)+\sum_{q \geq 0} \varphi\left(2^{-q} t\right)=1 .
$$

Définissons aussi l'opérateur :

$$
S_{q}^{\mathrm{v}} u=\sum_{q^{\prime} \leq q-1} \triangle_{q^{\prime}}^{\mathrm{v}} u .
$$


L'intérêt d'une telle décomposition réside dans le fait que la dérivation verticale d'une fonction localisée en fréquences verticales de taille $2^{q}$ va agir comme la multiplication par $2^{q}$. Aussi, le passage d'une norme Lebesgue sur une fonction localisée en fréquences verticales à une autre norme Lebesgue, peut se compter par la multiplication avec la taille de la troncature en fréquences à une certaine puissance dépendant des normes envisagées. Nous utilisons constamment le lemme suivant. Les estimations (3.3) et (3.4) sont des inégalités de type Bernstein.

Lemme 3.2 Soit $u$ une fonction avec supp $\mathcal{F}^{\mathrm{v}} u \subset \mathbb{R}_{h}^{2} \times 2^{q} \mathcal{C}$, où l'on a noté par $\mathcal{F}^{\mathrm{v}}$ la transformation de Fourier dans la variable verticale et où $\mathcal{C}$ est une couronne dyadique. Soient $p \geq 1$ et $r \geq r^{\prime} \geq 1$, nombres réels. On $a$ :

$$
\begin{aligned}
2^{q k} C^{-k}\|u\|_{L_{h}^{p}\left(L_{\mathrm{v}}^{r}\right)} & \leq\left\|\partial_{x_{3}}^{k} u\right\|_{L_{h}^{p}\left(L_{\mathrm{v}}^{r}\right)} \leq 2^{q k} C^{k}\|u\|_{L_{h}^{p}\left(L_{\mathrm{v}}^{r}\right)} \\
2^{q k} C^{-k}\|u\|_{L_{\mathrm{v}}^{r}\left(L_{h}^{p}\right)} & \leq\left\|\partial_{x_{3}}^{k} u\right\|_{L_{\mathrm{v}}^{r}\left(L_{h}^{p}\right)} \leq 2^{q k} C^{k}\|u\|_{L_{\mathrm{v}}^{r}\left(L_{h}^{p}\right)} \\
\|u\|_{L_{h}^{p}\left(L_{\mathrm{v}}^{r}\right)} & \leq C 2^{q\left(\frac{1}{r^{\prime}}-\frac{1}{r}\right)}\|u\|_{L_{h}^{p}\left(L_{\mathrm{v}}^{r^{\prime}}\right)} \\
\|u\|_{L_{\mathrm{v}}^{r}\left(L_{h}^{p}\right)} & \leq C 2^{q\left(\frac{1}{r^{\prime}}-\frac{1}{r}\right)}\|u\|_{L_{\mathrm{v}}^{r^{\prime}\left(L_{h}^{p}\right)}} .
\end{aligned}
$$

Démonstration. Soient $\tilde{\mathcal{C}}$ une couronne qui contient la couronne $\mathcal{C}$ et $\phi$ une fonction régulière à support compact inclus dans la couronne $\tilde{\mathcal{C}}$, telle que $\phi$ vaut 1 sur la couronne $\mathcal{C}$. Notons par $h$ la fonction rapidement décroissante telle que $\mathcal{F}^{\mathrm{v}} h=\phi$. Parce que supp $\mathcal{F}^{\mathrm{v}} u \subset 2^{q} \mathcal{C}$, on a

$$
\mathcal{F}^{\mathrm{v}} u\left(x_{h}, \xi_{3}\right)=\phi\left(\xi_{3} / 2^{q}\right) \mathcal{F}^{\mathrm{v}} u\left(x_{h}, \xi_{3}\right),
$$

donc

$$
\begin{aligned}
u\left(x_{h}, x_{3}\right)=2^{q} h\left(2^{q} \cdot\right) *_{x_{3}} u\left(x_{h}, \cdot\right)\left(x_{3}\right) & =2^{q} \int h\left(2^{q}\left(x_{3}-y_{3}\right)\right) u\left(x_{h}, y_{3}\right) d y_{3} \\
& =\int h\left(y_{3}\right) u\left(x_{h}, x_{3}-2^{-q} y_{3}\right) d y_{3} .
\end{aligned}
$$

Nous avons ainsi

$$
\begin{aligned}
\mathcal{F}^{\mathrm{v}} \partial_{x_{3}}^{k} u\left(x_{h}, \xi_{3}\right)=\xi_{3}^{k} \phi\left(\frac{\xi_{3}}{2^{q}}\right) \mathcal{F}^{\mathrm{v}} u\left(x_{h}, \xi_{3}\right) & =2^{q k}\left(\frac{\xi_{3}}{2^{q}}\right)^{k} \phi\left(\frac{\xi_{3}}{2^{q}}\right) \mathcal{F}^{\mathrm{v}} u\left(x_{h}, \xi_{3}\right) \\
& =2^{q k} \phi_{k}\left(\frac{\xi_{3}}{2^{q}}\right) \mathcal{F}^{\mathrm{v}} u\left(x_{h}, \xi_{3}\right)
\end{aligned}
$$

où $\phi_{k}\left(\xi_{3}\right)=\xi_{3}^{k} \phi\left(\xi_{3}\right) \in \mathrm{C}_{\mathrm{o}}^{\infty}(\mathbb{R})$. 
Parce que $\mathcal{F}^{\mathrm{v}} h^{(k)}\left(\xi_{3}\right)=\xi_{3}^{k} \mathcal{F}^{\mathrm{v}} h\left(\xi_{3}\right)=\xi_{3}^{k} \phi\left(\xi_{3}\right)$, on a :

$$
\begin{aligned}
\partial_{3}^{k} u\left(x_{h}, x_{3}\right) & =2^{q k} 2^{q} h^{(k)}\left(2^{q} \cdot\right) *_{x_{3}} u\left(x_{h}, \cdot\right)\left(x_{3}\right) \\
& =2^{q k} \int h^{(k)}\left(y_{3}\right) u\left(x_{h}, x_{3}-2^{-q} y_{3}\right) d y_{3} .
\end{aligned}
$$

Dans la suite, on utilisera souvent l'inégalité de convolution classique

$$
\|f * g\|_{L^{c}} \leq\|f\|_{L^{a}}\|g\|_{L^{b}} \quad \text { où } \quad 1+\frac{1}{c}=\frac{1}{a}+\frac{1}{b} .
$$

En prenant la norme $L_{\mathrm{v}}^{r}$ dans l'identité (3.5) et en utilisant l'inégalité de convolution (3.6) avec $c=b=r$ et $a=1$, on obtient

$$
\left\|\partial_{3}^{k} u\left(x_{h}, \cdot\right)\right\|_{L_{\mathrm{v}}^{r}} \leq 2^{q k} C\left\|u\left(x_{h}, \cdot\right)\right\|_{L_{\mathrm{v}}^{r}} .
$$

En appliquant maintenant la norme $L_{h}^{p}$, on obtient

$$
\left\|\partial_{3}^{k} u\right\|_{L_{h}^{p}\left(L_{\mathrm{v}}^{r}\right)} \leq C 2^{q k}\|u\|_{L_{h}^{p}\left(L_{\mathrm{v}}^{r}\right)} .
$$

On peut faire de la même manière dans le cas de la norme $L_{\mathrm{v}}^{r}\left(L_{h}^{p}\right)$. En appliquant la norme $L_{h}^{p}$ dans l'identité

$$
\partial_{3}^{k} u\left(x_{h}, x_{3}\right)=2^{q k} \int 2^{q} h^{(k)}\left(2^{q}\left(x_{3}-y_{3}\right)\right) u\left(x_{h}, y_{3}\right) d y_{3},
$$

on obtient

$$
\left\|\partial_{3}^{k} u\left(\cdot, x_{3}\right)\right\|_{L_{h}^{p}} \leq 2^{q k} \int 2^{q}\left|h^{(k)}\left(2^{q}\left(x_{3}-y_{3}\right)\right)\right|\left\|u\left(\cdot, y_{3}\right)\right\|_{L_{h}^{p}} d y_{3} .
$$

En appliquant maintenant la norme $L_{\mathrm{v}}^{r}$ sur cette inégalité et en utilisant l'inégalité de convolution (3.6) avec $c=b=r$ et $a=1$, on a :

$$
\left\|\partial_{3}^{k} u\right\|_{L_{\mathrm{v}}^{r}\left(L_{h}^{p}\right)} \leq 2^{q k} C\|u\|_{L_{\mathrm{v}}^{r}\left(L_{h}^{p}\right)} .
$$

Parce que le support de $\mathcal{F}^{\mathrm{v}} u$ en variable verticale est inclus dans la couronne $2^{q} \mathcal{C}$, on a

$$
\mathcal{F}^{\mathrm{v}} u\left(x_{h}, \xi_{3}\right)=\frac{\phi\left(2^{-q} \xi_{3}\right)}{\xi_{3}^{k}} \xi_{3}^{k} \mathcal{F}^{\mathrm{v}} u\left(x_{h}, \xi_{3}\right)
$$

Ainsi donc, nous écrivons

$$
\begin{aligned}
\mathcal{F}^{\mathrm{v}} u\left(x_{h}, \xi_{3}\right)=\frac{\phi\left(2^{-q} \xi_{3}\right)}{\xi_{3}^{k}} \mathcal{F}^{\mathrm{v}}\left(\partial_{3}^{k} u\right)\left(x_{h}, \xi_{3}\right) & =2^{-q k} \frac{\phi\left(\xi_{3} / 2^{q}\right)}{\left(\xi_{3} / 2^{q}\right)^{k}} \mathcal{F}^{\mathrm{v}}\left(\partial_{3}^{k} u\right)\left(x_{h}, \xi_{3}\right) \\
& =2^{-q k} \psi\left(\xi_{3} / 2^{q}\right) \mathcal{F}^{\mathrm{v}}\left(\partial_{3}^{k} u\right)\left(x_{h}, \xi_{3}\right),
\end{aligned}
$$


et en tenant compte du fait que

$$
\psi\left(\xi_{3}\right)=\frac{\phi\left(\xi_{3}\right)}{\xi_{3}^{k}} \in \mathrm{C}_{\mathrm{o}}^{\infty}(\mathbb{R}),
$$

nous obtenons :

$$
u\left(x_{h}, x_{3}\right)=2^{-q k} \tilde{h}_{q}() *_{x_{3}} \partial_{3}^{k} u\left(x_{h}, \cdot\right)\left(x_{3}\right),
$$

où $\tilde{h}$ est la fonction à décroissance rapide telle que $\mathcal{F}^{\mathrm{v}} \tilde{h}=\psi$ et où on a noté $\tilde{h}_{q}\left(y_{3}\right)=2^{q} \tilde{h}\left(2^{q} y_{3}\right)$.

Comme auparavant, nous obtenons

$$
\|u\|_{L_{h}^{p}\left(L_{\mathrm{v}}^{r}\right)} \leq 2^{-q k} C\left\|\partial_{3}^{k} u\right\|_{L_{h}^{p}\left(L_{\mathrm{v}}^{r}\right)}
$$

et aussi

$$
\|u\|_{L_{\mathrm{v}}^{r}\left(L_{h}^{p}\right)} \leq 2^{-q k} C\left\|\partial_{3}^{k} u\right\|_{L_{\mathrm{v}}^{r}\left(L_{h}^{p}\right)} .
$$

Pour démontrer les deux assertions suivantes, on écrit

$$
\mathcal{F}^{\mathrm{v}} u\left(x_{h}, \xi_{3}\right)=\phi\left(\xi_{3} / 2^{q}\right) \mathcal{F}^{\mathrm{v}} u\left(x_{h}, \xi_{3}\right)
$$

ce qui implique

$$
u\left(x_{h}, x_{3}\right)=2^{q} h\left(2^{q} \cdot\right) *_{x_{3}} u\left(x_{h}, \cdot\right)\left(x_{3}\right)=\int 2^{q} h\left(2^{q}\left(x_{3}-y_{3}\right)\right) u\left(x_{h}, y_{3}\right) d y_{3} .
$$

En prenant d'abord la norme $L_{h}^{p}$, on a l'inégalité

$$
\left\|u\left(\cdot, x_{3}\right)\right\|_{L_{h}^{p}} \leq \int 2^{q}\left|h\left(2^{q}\left(x_{3}-y_{3}\right)\right)\right| \cdot\left\|u\left(\cdot, y_{3}\right)\right\|_{L_{h}^{p}} d y_{3} .
$$

En appliquant maintenant la norme $L_{\mathrm{v}}^{r}$ et en utilisant l'inégalité de convolution (3.6), on obtient :

$$
\|u\|_{L_{\mathbf{v}}^{r}\left(L_{h}^{p}\right)} \leq\left\|2^{q} h\left(2^{q} \cdot\right)\right\|_{L^{s}}\|u\|_{L_{\mathbf{v}}^{r^{\prime}\left(L_{h}^{p}\right)}} \text { avec } \frac{1}{s}=\frac{1}{r}-\frac{1}{r^{\prime}}+1,
$$

nous obtenons ainsi :

$$
\|u\|_{L_{\mathrm{v}}^{r}\left(L_{h}^{p}\right)} \leq 2^{q\left(1 / r^{\prime}-1 / r\right)} C\|u\|_{L_{\mathrm{v}}^{r^{\prime}\left(L_{h}^{p}\right)}} .
$$

On procède de la même manière pour les normes $L_{h}^{p}\left(L_{\mathrm{v}}^{r}\right)$. Ceci achève la démonstration du lemme 3.2 .

Nous allons utiliser aussi le lemme suivant. La première inégalité est une estimation de type "Gagliardo-Nirenberg". Le deuxième point du lemme, est une estimation sur le commutateur avec l'opérateur de localisation en fréquences. 
Lemme 3.3 Pour toute fonction $u$, nous avons les estimations suivantes

$$
\|u\|_{L^{2}\left(\mathbb{R}_{x_{3}} ; L^{4}\left(\mathbb{R}_{x_{1}} \times \mathbb{R}_{x_{2}}\right)\right)} \leq C\|u\|_{L^{2}\left(\mathbb{R}^{3}\right)}^{1 / 2}\left\|\nabla_{h} u\right\|_{L^{2}\left(\mathbb{R}^{3}\right)}^{1 / 2} .
$$

Soient $p \geq 1, r \geq 1$ et $s \geq 1$ nombres réels tels que $\frac{1}{p}=\frac{1}{r}+\frac{1}{s}$. Alors

$$
\left\|\left[\triangle_{q}^{\mathrm{v}} ; u\right] v\right\|_{L_{\mathrm{v}}^{2}\left(L_{h}^{p}\right)} \leq 2^{-q} C\left\|\partial_{3} u\right\|_{L_{\mathrm{v}}^{\infty}\left(L_{h}^{r}\right)}\|v\|_{L_{\mathrm{v}}^{2}\left(L_{h}^{s}\right)} .
$$

Démonstration. Pour le premier point de ce lemme, on utilise le fait que l'espace d'interpolation entre $L^{2}\left(\mathbb{R}_{x_{1}} \times \mathbb{R}_{x_{2}}\right)$ et $\dot{H}^{1}\left(\mathbb{R}_{x_{1}} \times \mathbb{R}_{x_{2}}\right)$ est $\dot{H}^{1 / 2}\left(\mathbb{R}_{x_{1}} \times\right.$ $\left.\mathbb{R}_{x_{2}}\right)$, et on a l'inclusion continue de $\dot{H}^{1 / 2}\left(\mathbb{R}_{x_{1}} \times \mathbb{R}_{x_{2}}\right)$ dans $L^{4}\left(\mathbb{R}_{x_{1}} \times \mathbb{R}_{x_{2}}\right)$. Ceci revient à :

$$
\begin{aligned}
\left\|u\left(\cdot, x_{3}\right)\right\|_{L^{4}\left(\mathbb{R}_{x_{1}} \times \mathbb{R}_{x_{2}}\right)} & \leq C\left\|u\left(\cdot, x_{3}\right)\right\|_{\dot{H}^{1 / 2}\left(\mathbb{R}_{x_{1}} \times \mathbb{R}_{x_{2}}\right)} \\
& \leq C\left\|u\left(\cdot, x_{3}\right)\right\|_{L^{2}\left(\mathbb{R}_{x_{1}} \times \mathbb{R}_{x_{2}}\right)}^{1 / 2}\left\|\nabla_{h} u\left(\cdot, x_{3}\right)\right\|_{L^{2}\left(\mathbb{R}_{x_{1}} \times \mathbb{R}_{x_{2}}\right)}^{1 / 2} .
\end{aligned}
$$

En prenant maintenant la norme $L^{2}$ en $x_{3}$ on obtient :

$$
\|u\|_{L^{2}\left(\mathbb{R}_{x_{3}} ; L^{4}\left(\mathbb{R}_{x_{1}} \times \mathbb{R}_{x_{2}}\right)\right)} \leq C\|u\|_{L^{2}\left(\mathbb{R}^{3}\right)}^{1 / 2}\left\|\nabla_{h} u\right\|_{L^{2}\left(\mathbb{R}^{3}\right)}^{1 / 2} .
$$

L'estimation (3.8) sur le commutateur se démontre comme suit

$$
\begin{aligned}
{\left[\triangle_{q}^{\mathrm{v}} ; u\right] v } & \left(x_{h} ; x_{3}\right)=2^{q} \int_{\mathbb{R}_{\mathrm{v}}} h\left(2^{q} y_{3}\right)\left(u\left(x_{h} ; x_{3}-y_{3}\right)-u\left(x_{h} ; x_{3}\right)\right) v\left(x_{h} ; x_{3}-y_{3}\right) d y_{3} \\
& =2^{q} \int_{\mathbb{R}_{\mathrm{v}} \times[0,1]} h\left(2^{q} y_{3}\right) y_{3} \partial_{3} u\left(x_{h} ; x_{3}+\tau\left(x_{3}-y_{3}\right)\right) v\left(x_{h} ; x_{3}-y_{3}\right) d y_{3} d \tau \\
& =\int_{\mathbb{R}_{\mathrm{v}} \times[0,1]} \tilde{h}\left(2^{q} y_{3}\right) \partial_{3} u\left(x_{h} ; x_{3}+\tau\left(x_{3}-y_{3}\right)\right) v\left(x_{h} ; x_{3}-y_{3}\right) d y_{3} d \tau
\end{aligned}
$$

où $\tilde{h}(z)=h(z) \cdot z$.

À l'aide de l'inégalité de Hölder sur la variable horizontale, on a :

$$
\begin{aligned}
&\left\|\left[\triangle_{q}^{\mathrm{v}} ; u\right] v\right\|_{L^{p}\left(\mathbb{R}_{h}^{2}\right)} \leq C \int_{\mathbb{R}_{\mathrm{v}} \times[0,1]}\left|\tilde{h}\left(2^{q} y_{3}\right)\right| \| \partial_{3} u\left(x_{3}\right.\left.+\tau\left(x_{3}-y_{3}, \cdot\right)\right) \|_{L_{h}^{r}} \times \\
& \times\left\|v\left(x_{3}-y_{3}, \cdot\right)\right\|_{L_{h}^{s}} d y_{3} d \tau \\
& \leq C\left\|\partial_{3} u\right\|_{L_{\mathrm{v}}^{\infty}\left(L_{h}^{r}\right)} \int_{\mathbb{R}_{\mathrm{v}} \times[0,1]}\left|\tilde{h}\left(2^{q} y_{3}\right)\right|\left\|v\left(x_{3}-y_{3}, \cdot\right)\right\|_{L_{h}^{s}} d y_{3} d \tau .
\end{aligned}
$$

D'où il vient

$$
\left\|\left[\triangle_{q}^{\mathrm{v}} ; u\right] v\right\|_{L_{\mathrm{v}}^{2}\left(L_{h}^{p}\right)} \leq C 2^{-q}\left\|\partial_{3} u\right\|_{L_{\mathrm{v}}^{\infty}\left(L_{h}^{r}\right)}\|v\|_{L_{\mathrm{v}}^{2}\left(L_{h}^{s}\right)} .
$$

Nous avons maintenant les corollaires suivants : 
Corollaire 1 Si u est telle que le support de $\mathcal{F}^{\mathrm{v}} u$ est inclus dans $\mathbb{R}_{h}^{2} \times 2^{q} \mathcal{C}$, alors on a

$$
\|u\|_{L^{4}\left(\mathbb{R}_{x_{1}} \times \mathbb{R}_{x_{2}} ; L^{\infty}\left(\mathbb{R}_{x_{3}}\right)\right)} \leq C 2^{q / 2}\|u\|_{L^{2}\left(\mathbb{R}^{3}\right)}^{1 / 2}\left\|\nabla_{h} u\right\|_{L^{2}\left(\mathbb{R}^{3}\right)}^{1 / 2} .
$$

Démonstration. On tient compte du fait que $u$ est localisée en fréquences verticales de taille $2^{q}$, et on obtient :

$$
\|u\|_{L_{h}^{4}\left(L_{\mathrm{v}}^{\infty}\right)} \leq C 2^{q / 2}\|u\|_{L_{h}^{4}\left(L_{\mathrm{v}}^{2}\right)} \leq C 2^{q / 2}\|u\|_{L_{\mathrm{v}}^{2}\left(L_{h}^{4}\right)} \leq C 2^{q / 2}\|u\|_{L^{2}}^{1 / 2}\left\|\nabla_{h} u\right\|_{L^{2}}^{1 / 2} .
$$

Corollaire $\mathbf{2}$ On a les inégalités :

$$
\begin{aligned}
\|u\|_{L_{h}^{2}\left(L_{\mathrm{v}}^{\infty}\right)} & \leq C\|u\|_{\mathcal{B}^{0,1 / 2}} \\
\|u\|_{L_{h}^{4}\left(L_{\mathrm{v}}^{\infty}\right)} & \leq C\|u\|_{\mathcal{B}^{0,1 / 2}}^{1 / 2}\left\|\nabla_{h} u\right\|_{\mathcal{B}^{0,1 / 2}}^{1 / 2}
\end{aligned}
$$

Démonstration. Pour démontrer le premier point, écrivons que

$$
\|u\|_{L_{h}^{2}\left(L_{\mathrm{v}}^{\infty}\right)} \leq \sum_{q \in \mathbb{Z}}\left\|\triangle_{q}^{\mathrm{v}} u\right\|_{L_{h}^{2}\left(L_{\mathrm{v}}^{\infty}\right)} \leq C \sum_{q \in \mathbb{Z}} 2^{q / 2}\left\|\triangle_{q}^{\mathrm{v}} u\right\|_{L^{2}} \leq C\|u\|_{\mathcal{B}^{0,1 / 2}} .
$$

Pour le deuxième point, utilisons d'abord que

$$
\|u\|_{L_{h}^{4}\left(L_{\mathrm{v}}^{\infty}\right)} \leq \sum_{q \in \mathbb{Z}}\left\|\triangle_{q}^{\mathrm{v}} u\right\|_{L_{h}^{4}\left(L_{\mathrm{v}}^{\infty}\right)} \leq C \sum_{q \in \mathbb{Z}} 2^{q / 2}\left\|\triangle_{q}^{\mathrm{v}} u\right\|_{L^{2}}^{1 / 2}\left\|\nabla_{h} \triangle_{q}^{\mathrm{v}} u\right\|_{L^{2}}^{1 / 2} .
$$

En utilisant maintenant l'inégalité de Cauchy-Schwartz, on obtient :

$$
\begin{aligned}
\|u\|_{L_{h}^{4}\left(L_{\mathrm{v}}^{\infty}\right)} & \leq C\left(\sum_{q \in \mathbb{Z}} 2^{q / 2}\left\|\triangle_{q}^{\mathrm{v}} u\right\|_{L^{2}}\right)^{1 / 2}\left(\sum_{q \in \mathbb{Z}} 2^{q / 2}\left\|\nabla_{h} \triangle_{q}^{\mathrm{v}} u\right\|_{L^{2}}\right)^{1 / 2} \\
& =C\|u\|_{\mathcal{B}^{0,1 / 2}}^{1 / 2}\left\|\nabla_{h} u\right\|_{\mathcal{B}^{0,1 / 2}}^{1 / 2} .
\end{aligned}
$$

\section{Calcul paradifférentiel anisotrope.}

La décomposition dyadique est utile aussi dans l'étude du produit de deux distributions. Formellement, on peut écrire, pour deux distributions $u$ et $v$,

$$
u=\sum_{q \in \mathbb{Z}} \triangle_{q}^{\mathrm{v}} u \quad ; \quad v=\sum_{q \in \mathbb{Z}} \triangle_{q}^{\mathrm{v}} v \quad \text { et } \quad u \cdot v=\sum_{q \in \mathbb{Z}, q^{\prime} \in \mathbb{Z}} \triangle_{q}^{\mathrm{v}} u \cdot \triangle_{q^{\prime}}^{\mathrm{v}} v .
$$

On va séparer cette somme en trois sommes, l'une où les fréquences verticales de $u$ sont petites devant les fréquences verticales de $v$, une autre où les fréquences verticales de $v$ sont petites devant les fréquences verticales de $u$, et la dernière somme, où les fréquences de $u$ et de $v$ sont comparables. 
Les premières deux sommes sont les paraproduits et la dernière est le reste. Cette décomposition s'appelle la décomposition de Bony dans la variable verticale (voir [1] respectivement [6] et [11] pour le cas anisotrope).

Nous allons noter :

$$
T_{u} v=\sum_{q^{\prime} \leq q-2} \triangle_{q^{\prime}}^{\mathrm{v}} u \cdot \triangle_{q}^{\mathrm{v}} v=\sum_{q} S_{q-1}^{\mathrm{v}} u \cdot \triangle_{q}^{\mathrm{v}} v
$$

et

$$
R(u, v)=\sum_{q} \sum_{i \in\{0, \pm 1\}} \triangle_{q}^{\mathrm{v}} u \triangle_{q+i}^{\mathrm{v}} v
$$

Remarquons qu'on a des bonnes propriétés de quasi-orthogonalité dans le sens

$$
\begin{array}{llll}
\triangle_{q}^{\mathrm{v}}\left(S_{q^{\prime}-1}^{\mathrm{v}} u \triangle_{q^{\prime}}^{\mathrm{v}} v\right) & =0 & \text { pour } & \left|q-q^{\prime}\right| \geq 5 \\
\triangle_{q}^{\mathrm{v}}\left(S_{q^{\prime}+1}^{\mathrm{v}} u \triangle_{q^{\prime}}^{\mathrm{v}} v\right) & =0 & \text { et aussi }
\end{array}
$$

Pour alléger les démonstrations qui s'appuient sur le calcul paradifférentiel, nous allons noter par $a_{q}$ une suite génerique qui est de racine carrée sommable, c'est-à-dire, $a_{q} \geq 0$ et

$$
\sum_{q \in \mathbb{Z}} \sqrt{a}_{q} \leq 1
$$

On va désigner par $b_{q} \geq 0$ une suite générique sommable et par $c_{q}$ une suite de carré sommable telle que

$$
\sum_{q \in \mathbb{Z}} b_{q} \leq 1 \quad \text { et } \quad \sum_{q \in \mathbb{Z}} c_{q}^{2} \leq 1
$$

Signalons que les suites peuvent dépendre du paramètre $t$ et alors on note $a_{q}(t), b_{q}(t)$ et $c_{q}(t)$.

Notons ici, que l'appartenance d'une fonction $u$ à l'espace $\mathcal{B}^{0,1 / 2}$ se traduit par l'existence d'une suite $b_{q}$, telle que

$$
\left\|\triangle_{q}^{\mathrm{v}} u\right\|_{L^{2}} \leq C 2^{-q / 2} b_{q}\|u\|_{\mathcal{B}^{0,1 / 2}}
$$

Dans le même esprit, notons que $u$ appartient à l'espace de Sobolev $H^{0, s}$ si et seulement si

$$
\left\|\triangle_{q}^{\mathrm{v}} u\right\|_{L^{2}} \leq C 2^{-q s} c_{q}\|u\|_{H^{0, s}} .
$$

Dans la suite, il sera utile d'introduire des espaces de type Besov pour des champs de vecteurs dépendant du temps. Ces espaces prennent en compte la régularité Lebesgue en temps sur les blocs dyadiques verticaux. La définition 
de ces espaces s'inspire de l'article [5]. Notons ainsi par $\widetilde{L_{T}^{p}}\left(\mathcal{B}^{0,1 / 2}\right)$ avec $p \geq 1$, l'espace défini par la norme :

$$
\|u\|_{\widetilde{L_{T}^{p}\left(\mathcal{B}^{0,1 / 2}\right)}}=\sum_{q \in \mathbb{Z}} 2^{q / 2}\left\|\triangle_{q}^{\mathrm{v}} u\right\|_{L^{p}\left([0, T] ; L^{2}\left(\mathbb{R}^{3}\right)\right)}<+\infty .
$$

Observons que pour tout $p \geq 1$, on a $\|u\|_{L_{T}^{p}\left(\mathcal{B}^{0,1 / 2}\right)} \leq\|u\|_{{\widetilde{L_{T}^{p}}}_{\left(\mathcal{B}^{0,1 / 2}\right)}}$.

\section{Estimations d'énergie et démonstration du théorème}

\subsection{Estimations d'énergie pour l'équation de Navier-Stokes}

La démonstration de l'existence locale pour l'équation de Navier-Stokes est basée sur des estimations d'énergie sur le terme convectif $v \nabla v$. L'estimation d'énergie que nous allons utiliser est semblable à l'estimation d'énergie donnée dans [6], mais dans le cadre des espaces $\widetilde{L_{T}^{p}}\left(\mathcal{B}^{0,1 / 2}\right)$.

Lemme 4.1 (estimation pour l'existence locale) Soient $u$ et $v$ deux champs de vecteurs dépendant du temps sur $\mathbb{R}^{3}$, avec $u(t)$ de divergence nulle pour tout $t \in[0, T](T>0)$. Il existe une suite de nombres réels $a_{q} \geq 0$, avec $\sum_{q \in \mathbb{Z}} a_{q}^{1 / 2} \leq 1$, qui dépendent de $u$, $v$ et $T$, telle que

$$
\begin{aligned}
& \int_{0}^{T}\left|\left(\triangle_{q}^{\mathrm{v}}(u \cdot \nabla v) \mid \triangle_{q}^{\mathrm{v}} v\right)_{L^{2}}\right| d t \leq C a_{q} 2^{-q}\left(\|u\|_{L_{T}^{\infty}\left(\mathcal{B}^{0,1 / 2}\right)}^{\frac{1}{2}}\left\|\nabla_{h} u\right\|_{\widetilde{L_{T}^{2}}\left(\mathcal{B}^{0,1 / 2}\right)}^{\frac{1}{2}} \times\right. \\
& \left.\times\|v\|_{\frac{1}{L_{T}^{\infty}}\left(\mathcal{B}^{0,1 / 2}\right)}\left\|\nabla_{h} v\right\|_{\widetilde{L_{T}^{2}\left(\mathcal{B}^{0,1 / 2}\right)}}^{\frac{3}{2}}+\left\|\nabla_{h} u\right\|_{\widetilde{L_{T}^{2}\left(\mathcal{B}^{0,1 / 2}\right)}}\|v\|_{\widetilde{L_{T}^{\infty}}\left(\mathcal{B}^{0,1 / 2}\right)}\left\|\nabla_{h} v\right\|_{\widetilde{L_{T}^{2}\left(\mathcal{B}^{0,1 / 2}\right)}}\right),
\end{aligned}
$$

où $C>0$ est une constante universelle.

En particulier, on utilisera cette estimation dans le cas où $u=v$, dans la démonstration de l'existence globale. Nous avons ainsi le corollaire suivant :

Corollaire 3 (estimation pour l'existence globale) Il existe une constante $C$ telle que, pour tout champ de vecteur $v$ de divergence nulle, on a

$$
\int_{0}^{T}\left|\left(\triangle_{q}(v \nabla v)(t) \mid \triangle_{q}^{\mathrm{v}} v(t)\right)_{L^{2}}\right| d t \leq C 2^{-q} a_{q}\left\|\nabla_{h} v\right\|_{\widetilde{L}_{T}^{2}\left(\mathcal{B}^{0,1 / 2}\right)}^{2}\|v\|_{\widetilde{L_{T}^{\infty}}\left(\mathcal{B}^{0,1 / 2}\right)}
$$

avec

$$
\sum_{q \in \mathbb{Z}} a_{q}^{1 / 2} \leq 1
$$


On donne à présent l'estimation nécessaire pour démontrer l'unicité.

Lemme 4.2 (estimation pour l'unicité) Soient $u$ et $v$ deux champs de divergence nulle, qui appartiennent à l'espace $L_{T}^{\infty}\left(H^{0,1 / 2}\right)$ et tels que $\nabla_{h} u$ et $\nabla_{h} v$ appartiennent à l'espace $L_{T}^{2}\left(H^{0,1 / 2}\right)$. Soit $w$ dans $L_{T}^{\infty}\left(H^{0,1 / 2}\right)$ avec $\nabla_{h} w$ appartenant à l'espace $L_{T}^{2}\left(H^{0,1 / 2}\right)$ vérifiant l'équation

$$
\left\{\begin{array}{l}
\partial_{t} w+u \cdot \nabla w+w \cdot \nabla v-\nu_{h} \Delta_{h} w=-\nabla p \\
\operatorname{div} w=0 .
\end{array}\right.
$$

Alors, pour tout $0<t<T$, si $\|w(t)\|_{H^{0,-1 / 2}} \leq e^{-1}$, on a l'estimation

$$
\begin{aligned}
\frac{d}{d t}\|w(t)\|_{H^{0,-1 / 2}}^{2} \leq C f(t)\|w(t)\|_{H^{0,-1 / 2}}^{2} & \left(1-\ln \|w(t)\|_{H^{0,-1 / 2}}^{2}\right) \times \\
& \times \ln \left(1-\ln \|w(t)\|_{H^{0,-1 / 2}}^{2}\right),
\end{aligned}
$$

où $f(t)$ est la fonction localement intégrable en temps donnée par

$$
\begin{aligned}
f(t)=(1 & \left.+\|u(t)\|_{H^{0,1 / 2}}^{2}+\|v(t)\|_{H^{0,1 / 2}}^{2}+\|w(t)\|_{H^{0,1 / 2}}^{2}\right) \\
& \times\left(1+\left\|\nabla_{h} u(t)\right\|_{H^{0,1 / 2}}^{2}+\left\|\nabla_{h} v(t)\right\|_{H^{0,1 / 2}}^{2}+\left\|\nabla_{h} w(t)\right\|_{H^{0,1 / 2}}^{2}\right) .
\end{aligned}
$$

Remarque 4.1 Remarquons que, motivé par l'unicité, nous n'avons consideré que le cas où $\|w(t)\|_{H^{0,-1 / 2}}$ est petite.

Dans le cas général où $\|w(t)\|_{H^{0,-1 / 2}}$ peut être grande, l'estimation doublement logarithmique qu'on peut obtenir devient

$$
\begin{aligned}
\frac{d}{d t}\|w(t)\|_{H^{0,-1 / 2}}^{2} \leq C f(t)\|w(t)\|_{H^{0,-1 / 2}}^{2} & \ln \left(1+e+\frac{1}{\|w(t)\|_{H^{0,-1 / 2}}^{2}}\right) \times \\
& \times \ln \left(\ln \left(1+e+\frac{1}{\|w(t)\|_{H^{0,-1 / 2}}^{2}}\right)\right) .
\end{aligned}
$$

\subsection{Démonstration du théorème 1}

\section{Existence globale pour des données petites}

Nous allons démontrer l'existence globale pour des données initiales petites. La démonstration s'appuiera sur le corollaire 3 . On va utiliser la méthode de Friedrichs, qui consiste en l'approximation du système $\left(N S_{\nu_{\mathrm{v}}=0}\right)$ par une troncature dans l'espace des fréquences. Définissons alors les opérateurs suivants :

$$
\begin{aligned}
J_{n} u & =\mathcal{F}^{-1}\left(\mathbf{1}_{B(0, n)} \mathcal{F} u(\xi)\right) \\
J_{n}^{\mathrm{v}} u & =\mathcal{F}^{-1}\left(\mathbf{1}_{\left\{\xi|| \xi_{3} \mid \leq n\right\}} \mathcal{F} u(\xi)\right) \text { et } \\
\tilde{J}_{n} u & =\left(J_{n}-J_{\frac{1}{n}}^{\mathrm{v}}\right) u .
\end{aligned}
$$

où on a noté par $\mathcal{F}$ la transformation de Fourier sur $\mathbb{R}^{3}$. 
Notons que dans le système $\left(N S_{\nu_{\mathrm{v}}=0}\right)$, la condition de divergence nulle sur $v$ nous donne le terme de pression. On obtient ainsi

$$
p=-\sum_{i, j} \Delta^{-1} \partial_{i} \partial_{j}\left(v^{i} v^{j}\right)
$$

Considérons alors, le système approximant suivant

$$
\left(N S_{\nu_{\mathrm{v}=0}}^{n}\right) \quad\left\{\begin{array}{l}
\partial_{t} v_{n}+\tilde{J}_{n}\left(\tilde{J}_{n} v_{n} \cdot \nabla \tilde{J}_{n} v_{n}\right)-\nu_{h} \tilde{J}_{n}\left(\partial_{x_{1}}^{2}+\partial_{x_{2}}^{2}\right) v_{n} \\
=\tilde{J}_{n} \nabla \sum_{i, j} \partial_{i} \partial_{j} \Delta^{-1}\left(\tilde{J}_{n} v_{n}^{i} \tilde{J}_{n} v_{n}^{j}\right) \\
\operatorname{div} v_{n}=0 \\
\left.v_{n}\right|_{t=0}=\tilde{J}_{n} v_{0} .
\end{array}\right.
$$

Comme il est clair que les opérateurs bilinéaires sont continus de $L^{2} \times L^{2}$ dans $L^{2}$ et comme la donnée initiale $v_{n}(0)=\tilde{J}_{n} v_{0}$ appartient à $L^{2}$ lorsque $v_{0}$ est dans l'espace homogène $\mathcal{B}^{0,1 / 2}$, alors le système $\left(N S_{\nu_{\mathrm{v}}=0}^{n}\right)$ devient une équation différentielle dans $L^{2}$. Grâce au théorème de Cauchy-Lipschitz, il existe une unique solution locale en temps à valeurs dans $L^{2}$. Notons par $T_{n}$ le temps de vie maximal pour l'unique solution $v_{n}$ du système $\left(N S_{\nu_{\mathrm{v}}=0}^{n}\right)$. Comme $\tilde{J}_{n}^{2}=\tilde{J}_{n}$, on a évidemment que $\tilde{J}_{n} v_{n}$ est aussi une solution pour $\left(N S_{\nu_{\mathrm{v}}=0}^{n}\right)$, alors, l'unicité de la solution implique que $v_{n}=\tilde{J}_{n} v_{n}$. Donc, on a que $v_{n}$ est solution du système suivant, qu'on va noter toujours par $\left(N S_{\nu_{\mathrm{v}}=0}^{n}\right)$

$$
\left(N S_{\nu_{\mathrm{v}=0}}^{n}\right)\left\{\begin{array}{l}
\partial_{t} v_{n}+\tilde{J}_{n}\left(v_{n} \cdot \nabla v_{n}\right)-\nu_{h}\left(\partial_{x_{1}}^{2}+\partial_{x_{2}}^{2}\right) v_{n}=\tilde{J}_{n} \nabla \sum_{i, j} \partial_{i} \partial_{j} \Delta^{-1}\left(v_{n}^{i} v_{n}^{j}\right) \\
\operatorname{div} v_{n}=0 \\
\left.v_{n}\right|_{t=0}=\tilde{J}_{n} v_{0} .
\end{array}\right.
$$

Puisque $\left(\tilde{J}_{n}\left(v_{n} \nabla v_{n}\right) \mid v_{n}\right)_{L^{2}}=0$, l'estimation d'énergie dans $L^{2}$ nous donne

$$
\frac{1}{2} \frac{d}{d t}\left\|v_{n}(t)\right\|_{L^{2}}^{2}+\nu_{h}\left\|\nabla_{h} v_{n}(t)\right\|_{L^{2}}^{2}=0
$$

Donc, $v_{n}(t)$ reste bornée sur l'intervalle maximal d'existence $\left[0, T_{n}\right)$, d'où on déduit facilement que $T_{n}=+\infty$.

Tout d'abord, on va démontrer une estimation globale en temps sur la solution $v_{n}$ dans l'espace $\mathcal{B}^{0,1 / 2}$. Ensuite, par un argument classique de compacité et par passage à la limite dans le système approximant $\left(N S_{\nu_{\mathrm{v}}=0}^{n}\right)$, on démontre à la fin l'existence globale de la solution de l'equation $\left(N S_{\nu_{\mathrm{v}}=0}\right)$. Afin d'alléger les notations, on "oublie" dorénavant l'indice $n$. On va appliquer l'opérateur $\triangle_{q}^{\mathrm{v}}$ à l'équation approximante $\left(N S_{\nu_{\mathrm{v}=0}}^{n}\right)$ et on va faire 
les estimations d'énergie dans $L^{2}$ sur chaque morceau $\triangle_{q}^{\mathrm{v}} v$, et ensuite, on va recoller les estimations. Nous obtenons ainsi par l'estimation d'énergie sur $\triangle_{q}^{\mathrm{v}} v$, l'inégalité suivante :

$$
\frac{1}{2} \frac{d}{d t}\left\|\triangle_{q}^{\mathrm{v}} v(t)\right\|_{L^{2}}^{2}+\nu_{h}\left\|\triangle_{q}^{\mathrm{v}} \nabla_{h} v(t)\right\|_{L^{2}}^{2} \leq\left|\left(\triangle_{q}^{\mathrm{v}}(v \cdot \nabla v)(t) \mid \triangle_{q}^{\mathrm{v}} v(t)\right)_{L^{2}}\right| .
$$

L'intégration en temps sur un intervalle de temps $[0, t]$ avec $t>0$ nous donne

$$
\begin{aligned}
\left\|\triangle_{q}^{\mathrm{v}} v(t)\right\|_{L^{2}}^{2}+2 \nu_{h} \int_{0}^{t} & \left\|\nabla_{h} \triangle_{q}^{\mathrm{v}} v(\tau)\right\|_{L^{2}}^{2} d \tau \leq \\
& \leq\left\|\triangle_{q}^{\mathrm{v}} v_{0}\right\|_{L^{2}}^{2}+2 \int_{0}^{t}\left|\left(\triangle_{q}^{\mathrm{v}}(v \cdot \nabla v)(\tau) \mid \triangle_{q}^{\mathrm{v}} v(\tau)\right)_{L^{2}}\right| d \tau
\end{aligned}
$$

En prenant le supremum en $t \in[0, T]$, on a

$$
\begin{aligned}
\left\|\triangle_{q}^{\mathrm{v}} v\right\|_{L_{T}^{\infty}\left(L^{2}\right)}^{2}+2 \nu_{h} & \left\|\nabla_{h} \triangle_{q}^{\mathrm{v}} v\right\|_{L_{T}^{2}\left(L^{2}\right)}^{2} \leq \\
& \leq\left\|\triangle_{q}^{\mathrm{v}} v_{0}\right\|_{L^{2}}^{2}+2 \int_{0}^{T}\left|\left(\triangle_{q}^{\mathrm{v}}(v \cdot \nabla v)(\tau) \mid \triangle_{q}^{\mathrm{v}} v(\tau)\right)_{L^{2}}\right| d \tau
\end{aligned}
$$

Nous allons utiliser maintenant le corollaire 3 pour obtenir que

$$
\begin{aligned}
\left\|\triangle_{q}^{\mathrm{v}} v\right\|_{L_{T}^{\infty}\left(L^{2}\right)}^{2}+2 \nu_{h} & \left\|\nabla_{h} \triangle_{q}^{\mathrm{v}} v\right\|_{L_{T}^{2}\left(L^{2}\right)}^{2} \leq \\
& \leq\left\|\triangle_{q}^{\mathrm{v}} v_{0}\right\|_{L^{2}}^{2}+2^{-q} a_{q} C\|v\|_{\widetilde{L_{T}^{\infty}}\left(\mathcal{B}^{0,1 / 2}\right)}\left\|\nabla_{h} v\right\|_{\widetilde{L_{T}^{2}}\left(\mathcal{B}^{0,1 / 2}\right)}^{2}
\end{aligned}
$$

avec $\sum_{q} \sqrt{a_{q}} \leq 1$. D'où, par l'équivalence entre $a^{2}+b^{2}$ et $(a+b)^{2}$ quand $a$ et $b$ sont des nombres réels positifs, il vient

$$
\begin{aligned}
2^{q / 2} \| \triangle_{q}^{\mathrm{v}} v & \left\|_{L_{T}^{\infty}\left(L^{2}\right)}+\sqrt{2} \nu_{h}^{1 / 2} 2^{q / 2}\right\| \nabla_{h} \triangle_{q}^{\mathrm{v}} v \|_{L_{T}^{2}\left(L^{2}\right)} \leq \\
& \leq 2^{q / 2} \sqrt{2}\left\|\triangle_{q}^{\mathrm{v}} v_{0}\right\|_{L^{2}}+a_{q}^{1 / 2} \sqrt{2} C^{1 / 2}\|v\|_{\widetilde{L_{T}^{\infty}}\left(\mathcal{B}^{0,1 / 2}\right)}^{1 / 2}\left\|\nabla_{h} v\right\|_{\widetilde{L_{T}^{2}}\left(\mathcal{B}^{0,1 / 2}\right)}
\end{aligned}
$$

Par sommation en $q$, nous obtenons

$$
\begin{aligned}
\|v\|_{\widetilde{L_{T}^{\infty}}\left(\mathcal{B}^{0,1 / 2}\right)}+\sqrt{2} \nu_{h}^{1 / 2} & \left\|\nabla_{h} v\right\|_{\widetilde{L_{T}^{2}\left(\mathcal{B}^{0,1 / 2}\right)}} \leq \\
& \leq \sqrt{2}\left\|v_{0}\right\|_{\mathcal{B}^{0,1 / 2}}+C^{1 / 2} \sqrt{2}\|v\|_{\widetilde{L_{T}^{\infty}}\left(\mathcal{B}^{0,1 / 2}\right)}\left\|\nabla_{h} v\right\|_{\widetilde{L_{T}^{2}\left(\mathcal{B}^{0,1 / 2}\right)}}
\end{aligned}
$$

et donc

$$
\begin{aligned}
\|v\|_{\widetilde{L_{T}^{\infty}}\left(\mathcal{B}^{0,1 / 2}\right)}^{2} & +2 \nu_{h}\left\|\nabla_{h} v\right\|_{\widetilde{L_{T}^{2}\left(\mathcal{B}^{0,1 / 2}\right)}}^{2} \leq \\
& \leq 4\left\|v_{0}\right\|_{\mathcal{B}^{0,1 / 2}}^{2}+4 C\|v\|_{\widetilde{L_{T}^{\infty}\left(\mathcal{B}^{0,1 / 2}\right)}}\left\|\nabla_{h} v\right\|_{\widetilde{L_{T}^{2}}\left(\mathcal{B}^{0,1 / 2}\right)}^{2}
\end{aligned}
$$


On peut alors en déduire l'existence globale lorsque la donnée initiale est petite par rapport à la viscosité horizontale. En effet, soit $c$ assez petit et $v_{0} \in$ $\mathcal{B}^{0,1 / 2}$ un champ de divergence nulle, tel que $\left\|v_{0}\right\|_{\mathcal{B}^{0,1 / 2}}<c \nu_{h}$ avec $c<1 /(8 C)$. Soit $v_{n}$ la solution régulière de l'équation approximante de l'équation de Navier-Stokes anisotrope, avec la donnée initiale régularisée $\left(\left.v_{n}\right|_{t=0}=\tilde{J}_{n} v_{0}\right)$. Soit alors $T_{n}^{*}$ le temps maximal sur lequel on a $\left\|v_{n}\right\|_{\widetilde{L_{T}^{\infty}\left(\mathcal{B}^{0,1 / 2}\right)}} \leq 2 c \nu_{h}$ pour tout $0<T<T_{n}^{*}$. À l'aide de l'estimation (4.2), on obtient

$$
\left\|v_{n}\right\|_{\overparen{L_{T}^{\infty}}\left(\mathcal{B}^{0,1 / 2}\right)} \leq 2\left\|v_{0}\right\|_{\mathcal{B}^{0,1 / 2}}<2 c \nu_{h}
$$

pour tout temps $T$ avec $0<T<T_{n}^{*}$.

$\mathrm{Vu}$ que la fonction $v_{n}$ est régulière en temps à valeurs dans $\mathcal{B}^{0,1 / 2}$, et donc que la fonction $T \leadsto\left\|v_{n}\right\|_{{\widetilde{L_{T}^{\infty}}}_{\left(\mathcal{B}^{0,1 / 2}\right)}}$ est continue, nous obtenons $T_{n}^{*}=+\infty$, et on a que la suite des solutions globales $v_{n}$ est bornée dans l'espace

$$
v_{n} \in \widetilde{L^{\infty}}\left(\mathbb{R}_{+}, \mathcal{B}^{0,1 / 2}\right) \quad \text { avec } \nabla_{h} v_{n} \in \widetilde{L^{2}}\left(\mathbb{R}_{+}, \mathcal{B}^{0,1 / 2}\right)
$$

En particulier, comme on a $S_{0}^{\mathrm{v}} v_{n} \in L_{\mathrm{v}}^{\infty} L_{h}^{2}$ et $\left(I-S_{0}^{\mathrm{v}}\right) v_{n} \in L^{2}$ on obtient que $\left(v_{n}\right)_{n \in \mathbb{N}}$ est une suite bornée dans l'espace $L^{\infty}\left(\mathbb{R}_{+} ; L_{l o c}^{2}\right)$ et en utilisant l'équation vérifiée par $v_{n}$, on obtient que

$$
\left(\partial_{t} v_{n}\right)_{n \in \mathbb{N}} \text { est suite bornée dans } L^{\infty}\left(\mathbb{R}_{+} ; H_{l o c}^{-N}\right)
$$

avec $N$ suffisamment grand. Comme conséquence du théorème d'ArzelaAscoli, on a qu'il existe une sous-suite de $v_{n}$ qu'on va persister à noter $v_{n}$, tel que $v_{n} \longrightarrow v$ dans $L_{l o c}^{\infty}\left(\mathbb{R}_{+} ; H_{l o c}^{-N}\right)$. Parce que $v_{n}$ est une suite bornée dans $L^{\infty}\left(\mathbb{R}_{+} ; L_{\text {loc }}^{2}\right)$, on obtient par interpolation que

$$
v_{n} \longrightarrow v \text { dans } L_{l o c}^{\infty}\left(\mathbb{R}_{+} ; H_{l o c}^{-\sigma}\right) \text { pour tout } \sigma>0 .
$$

$\mathrm{Vu}$ que $v_{n}$ est une suite bornée dans $L_{\text {loc }}^{2}\left(\mathbb{R}_{+} ; H_{l o c}^{\varepsilon}\right)$ pour tout $\varepsilon<1 / 2$, on obtient comme conséquence des lois de produits dans les espaces de Sobolev classiques que

$$
v_{n} \otimes v_{n} \longrightarrow v \otimes v \quad \text { dans } \quad L_{l o c}^{2}\left(H_{l o c}^{\varepsilon-\sigma-3 / 2}\right) \quad \text { lorsque } \sigma<\varepsilon .
$$

En particulier

$$
v_{n} \otimes v_{n} \longrightarrow v \otimes v \quad \text { dans } \quad \mathcal{D}^{\prime} .
$$

Par passage à la limite dans l'équation approximante $\left(N S_{\nu_{\mathrm{v}}=0}^{n}\right)$ on obtient une solution globale du système $\left(N S_{\nu_{v}=0}\right)$.

Remarque 4.2 On a obtenu la solution globale d'une régularité meilleure:

$$
v \in \widetilde{L^{\infty}}\left(\mathbb{R}_{+}, \mathcal{B}^{0,1 / 2}\right) \quad \text { avec } \quad \nabla_{h} v \in \widetilde{L^{2}}\left(\mathbb{R}_{+}, \mathcal{B}^{0,1 / 2}\right)
$$




\section{Continuité en temps de la solution}

Nous allons démontrer maintenant la continuité en temps de la solution. En effet, on va démontrer que la solution $v$ appartient en fait à l'espace $v \in C_{b}\left(\mathbb{R}_{+}, \mathcal{B}^{0,1 / 2}\right)$.

On va démontrer d'abord, que la fonction $\left\|\triangle_{q}^{\mathrm{v} v} v(t)\right\|_{L^{2}}$ est continue en temps. Pour cela, il suffit de vérifier que pour $q$ fixé, la fonction $\partial_{t}\left\|\triangle_{q}^{\mathrm{v}} v(t)\right\|_{L^{2}}^{2}$ appartient à l'espace $L^{1}([0, T])$. L'équation vérifiée par $\triangle_{q}^{\mathrm{v}} v$ est la suivante

$$
\partial_{t} \triangle_{q}^{\mathrm{v}} v=\nu_{h} \Delta_{h} \triangle_{q}^{\mathrm{v}} v-\operatorname{div}{ }_{h} \triangle_{q}^{\mathrm{v}}(v \otimes v)-\triangle_{q}^{\mathrm{v}} \partial_{3}(v \otimes v)+\triangle_{q}^{\mathrm{v}} \nabla p .
$$

On obtient facilement que $\left(\nu_{h} \Delta_{h} \triangle_{q}^{\mathrm{v}} v-\operatorname{div}_{h} \triangle_{q}^{\mathrm{v}}(v \otimes v)\right)$ appartient à l'espace $L^{2}\left([0, T] ; L_{\mathrm{v}}^{2}\left(\dot{H}_{h}^{-1}\right)\right)$. Comme, d'autre part on a que $\triangle_{q}^{\mathrm{v}} v$ appartient à $L^{2}\left([0, T] ; L_{\mathrm{v}}^{2} \dot{H}_{h}^{1}\right)$, on obtient

$$
\left(\nu_{h} \Delta_{h} \triangle_{q}^{\mathrm{v}} v-\operatorname{div}_{h}(v \otimes v) \mid \triangle_{q}^{\mathrm{v}} v\right)_{L^{2}\left(\mathbb{R}^{3}\right)} \in L^{1}([0, T]) .
$$

En tenant compte du fait que $v$ appartient à $L^{2}\left([0, T] ; L_{h}^{4} L_{\mathrm{v}}^{2}\right)$ on obtient que $\triangle_{q}^{\mathrm{v}} \partial_{3}(v \otimes v)$ appartient à $L^{1}\left([0, T] ; L_{h}^{2} L_{\mathrm{v}}^{1}\right)$. Comme, d'autre part on a que $v \in L^{\infty}\left([0, T] ; L_{h}^{2} L_{\mathrm{v}}^{\infty}\right)$, on obtient

$$
\left(\triangle_{q}^{\mathrm{v}} \partial_{3}(v \otimes v) \mid \triangle_{q}^{\mathrm{v} v}\right)_{L^{2}} \in L^{1}([0, T]) .
$$

On a obtenu finalement que $\partial_{t}\left\|\triangle_{q}^{\mathrm{v}} v(t)\right\|_{L^{2}}^{2}$ appartient à l'espace $L_{T}^{1}$ et en particulier, pour $q$ fixé, on a que $\left\|\triangle_{q}^{\mathrm{v}} v(t)\right\|_{L^{2}} \in C_{b}([0, T])$. D'autre part, il est très facile de démontrer que la fonction $t \leadsto \triangle_{q}^{\mathrm{v}} v(t)$ est faiblement continue. On obtient ainsi que $\triangle_{q}^{\mathrm{v}} v(t)$ est fonction fortement continue sur $[0, T]$ à valeurs dans $L^{2}$.

Le reste est simple. En effet, vu que $v \in \widetilde{L_{T}^{\infty}}\left(\mathcal{B}^{0,1 / 2}\right)$, pour $\varepsilon>0$, on a qu'il existe $N$ telle que

$$
\sum_{|q| \geq N} 2^{q / 2}\left\|\triangle_{q}^{\mathrm{v}} v\right\|_{L_{T}^{\infty}\left(L^{2}\right)} \leq \varepsilon
$$

Comme la fonction $\triangle_{q}^{\mathrm{v}} v \in C_{T}\left(L^{2}\right)$, il existe $\eta>0$ telle que

$$
\sum_{-N \leq q \leq N} 2^{q / 2}\left\|\triangle_{q}^{\mathrm{v}}\left(v(t)-v\left(t^{\prime}\right)\right)\right\|_{L^{2}} \leq \varepsilon \quad \text { pour } \quad\left|t-t^{\prime}\right| \leq \eta .
$$

On obtient alors que pour $\left|t-t^{\prime}\right| \leq \eta$ on a

$$
\left\|u(t)-u\left(t^{\prime}\right)\right\|_{\mathcal{B}^{0,1 / 2}} \leq \varepsilon \text {. }
$$

Nous obtenons ainsi la proposition suivante. 
Proposition 1 Soit $v \in \widetilde{L_{T}^{\infty}}\left(\mathcal{B}^{0,1 / 2}\right)$ une solution de l'équation $\left(N S_{\nu_{\mathrm{v}}=0}\right)$ sur l'intervalle de temps $[0, T)$ associée à la donnée initiale $v_{0} \in \mathcal{B}^{0,1 / 2}$. Alors on a

1. $v \in C_{b}\left([0, T), \mathcal{B}^{0,1 / 2}\right)$.

2. l'ensemble $\{v(t) \mid t \in[0, T)\}$ est un ensemble relativement compacte dans $\mathcal{B}^{0,1 / 2}$.

\section{Existence locale pour des données quelconques}

Pour démontrer l'existence locale pour des données quelconques, nous allons décomposer la donnée initiale en une partie "basses fréquences" et une partie "hautes fréquences". Nous allons chercher la solution locale de la forme $v=u_{N}+w_{N}$ avec

$$
u_{N}(t, x)=e^{t \nu_{h} \Delta_{h}}\left(S_{N} v_{0}\right)(x) .
$$

Observons que $u_{N}$ est solution du problème linéaire :

$$
\left\{\begin{array}{l}
\partial_{t} u_{N}-\nu_{h} \Delta_{h} u_{N}=0 \\
\left.u_{N}\right|_{t=0}=S_{N} v_{0}
\end{array}\right.
$$

Pour que $v$ soit solution de l'équation de Navier-Stokes, il faut que $w_{N}$ soit solution pour le système suivant :

$$
\left(S_{N}\right)\left\{\begin{array}{l}
\partial_{t} w_{N}+w_{N} \cdot \nabla w_{N}+u_{N} \cdot \nabla w_{N}+w_{N} \cdot \nabla u_{N}-\nu_{h} \Delta_{h} w_{N}= \\
\operatorname{div} w_{N}=0 \quad=-\nabla p-u_{N} \cdot \nabla u_{N} \\
\left.w_{N}\right|_{t=0}=\left(I-S_{N}\right) v_{0}
\end{array}\right.
$$

Pour alléger la démonstration, on omet de noter le schéma de Friedrichs. Nous allons démontrer que pour des temps assez petits, l'équation $\left(S_{N}\right)$ a une solution locale en temps. Faisons l'estimation d'énergie sur cette équation. On obtient :

$$
\begin{aligned}
\frac{1}{2} \frac{d}{d t}\left\|\triangle_{q}^{\mathrm{v}} w_{N}(t)\right\|_{L^{2}}^{2} & +\nu_{h}\left\|\nabla_{h} \triangle_{q}^{\mathrm{v}} w_{N}(t)\right\|_{L^{2}}^{2} \leq \\
\leq & \left|\left(\triangle_{q}^{\mathrm{v}}\left(w_{N} \nabla w_{N}\right) \mid \triangle_{q}^{\mathrm{v}} w_{N}\right)_{L^{2}}\right|+\left|\left(\triangle_{q}^{\mathrm{v}}\left(u_{N} \nabla w_{N}\right) \mid \triangle_{q}^{\mathrm{v}} w_{N}\right)_{L^{2}}\right| \\
& +\left|\left(\triangle_{q}^{\mathrm{v}}\left(w_{N} \nabla u_{N}\right) \mid \triangle_{q}^{\mathrm{v}} w_{N}\right)_{L^{2}}\right|+\left|\left(\triangle_{q}^{\mathrm{v}}\left(u_{N} \nabla u_{N}\right) \mid \triangle_{q}^{\mathrm{v}} w_{N}\right)_{L^{2}}\right| .
\end{aligned}
$$

Par le corollaire 3, nous avons :

$$
\int_{0}^{T}\left|\left(\triangle_{q}^{\mathrm{v}}\left(w_{N} \nabla w_{N}\right) \mid \triangle_{q}^{\mathrm{v}} w_{N}\right)\right| \leq C 2^{-q} a_{q}\left\|w_{N}\right\|_{\widetilde{L_{T}^{\infty}}\left(\mathcal{B}^{0,1 / 2}\right)}\left\|\nabla_{h} w_{N}\right\|_{\widetilde{L_{T}^{2}\left(\mathcal{B}^{0,1 / 2}\right)}}^{2}
$$

avec $a_{q} \in l_{q}^{1 / 2}$. 
Comme conséquence du lemme 4.1, nous avons :

$$
\begin{aligned}
& \int_{0}^{T}\left|\left(\triangle_{q}^{\mathrm{v}}\left(u_{N} \cdot \nabla w_{N}\right) \mid \triangle_{q}^{\mathrm{v}} w_{N}\right)_{L^{2}}\right| d \tau \leq \\
& \leq C 2^{-q} a_{q}\left(\left\|\nabla_{h} u_{N}\right\|_{\widetilde{L_{T}^{2}}\left(\mathcal{B}^{0,1 / 2}\right)}\left\|w_{N}\right\|_{\widetilde{L_{T}^{\infty}}\left(\mathcal{B}^{0,1 / 2}\right)}\left\|\nabla_{h} w_{N}\right\|_{\widetilde{L_{T}^{2}}\left(\mathcal{B}^{0,1 / 2}\right)}\right. \\
& \left.\quad+\left\|u_{N}\right\|_{\widetilde{L_{T}^{\infty}}\left(\mathcal{B}^{0,1 / 2}\right)}^{1 / 2}\left\|\nabla_{h} u_{N}\right\|_{\widetilde{L_{T}^{2}}\left(\mathcal{B}^{0,1 / 2}\right)}^{1 / 2}\left\|w_{N}\right\|_{\widetilde{L}_{T}^{\infty}\left(\mathcal{B}^{0,1 / 2}\right)}^{1 / 2}\left\|\nabla_{h} w_{N}\right\|_{\widetilde{L_{T}^{2}}\left(\mathcal{B}^{0,1 / 2}\right)}^{3 / 2}\right) .
\end{aligned}
$$

Pour le terme suivant, on a

$$
\begin{aligned}
\int_{0}^{T} \mid\left(\triangle_{q}^{\mathrm{v}}\left(w_{N} \cdot \nabla u_{N}\right) \mid\right. & \left.\triangle_{q}^{\mathrm{v}} w_{N}\right) \mid \leq\left\|\triangle_{q}^{\mathrm{v}}\left(w_{N} \cdot \nabla u_{N}\right)\right\|_{L_{T}^{1}\left(L^{2}\right)}\left\|\triangle_{q}^{\mathrm{v}} w_{N}\right\|_{L_{T}^{\infty}\left(L^{2}\right)} \\
& \leq 2^{-q / 2} b_{q} T\left\|\triangle_{q}^{\mathrm{v}}\left(w_{N} \nabla u_{N}\right)\right\|_{L_{T}^{\infty}\left(L^{2}\right)}\left\|w_{N}\right\|_{\widetilde{L_{T}^{\infty}}\left(\mathcal{B}^{0,1 / 2}\right)} .
\end{aligned}
$$

En utilisant la décomposition de Bony dans la variable verticale, nous écrivons $\triangle_{q}^{\mathrm{v}}\left(w_{N} \nabla u_{N}\right)=\triangle_{q}^{\mathrm{v}} \sum_{\left|q^{\prime}-q\right| \leq 4} S_{q^{\prime}-1}^{\mathrm{v}} w_{N} \cdot \triangle_{q^{\prime}}^{\mathrm{v}} \nabla u_{N}+\triangle_{q}^{\mathrm{v}} \sum_{q^{\prime} \geq q-N_{0}} S_{q^{\prime}+1}^{\mathrm{v}} \nabla u_{N} \cdot \triangle_{q^{\prime}}^{\mathrm{v}} w_{N}$.

On en déduit

$$
\begin{gathered}
\left\|\triangle_{q}^{\mathrm{v}}\left(w_{N} \cdot \nabla u_{N}\right)\right\|_{L_{T}^{\infty}\left(L^{2}\right)} \leq \sum_{\left|q^{\prime}-q\right| \leq 4}\left\|S_{q^{\prime}-1}^{\mathrm{v}} w_{N}\right\|_{L_{T}^{\infty}\left(L^{2}\right)}\left\|\triangle_{q^{\prime}}^{\mathrm{v}} \nabla u_{N}\right\|_{L_{T}^{\infty}\left(L^{\infty}\right)} \\
+\sum_{q^{\prime} \geq q-N_{0}}\left\|S_{q^{\prime}+1}^{\mathrm{v}} \nabla u_{N}\right\|_{L_{T}^{\infty}\left(L^{\infty}\right)}\left\|\triangle_{q^{\prime}}^{\mathrm{v}} w_{N}\right\|_{L_{T}^{\infty}\left(L^{2}\right)} \\
\leq\left\|w_{N}\right\|_{L_{T}^{\infty}\left(\mathcal{B}^{0,1 / 2}\right)} 2^{5 N / 2} \sum_{\left|q^{\prime}-q\right| \leq 4}\left\|\triangle_{q^{\prime}}^{\mathrm{v}} u_{N}\right\|_{L_{T}^{\infty}\left(L^{2}\right)} \\
+2^{5 N / 2}\left\|u_{N}\right\|_{L_{T}^{\infty}\left(L^{2}\right)} 2^{-q / 2}\left(2^{q / 2} \sum_{q^{\prime} \geq q-N_{0}} 2^{-q^{\prime} / 2} b_{q^{\prime}}\right)\left\|w_{N}\right\|_{\widetilde{L_{T}^{\infty}\left(\mathcal{B}^{0,1 / 2}\right)}}
\end{gathered}
$$

avec $b_{q^{\prime}} \in \ell^{1}$, ce qui nous donne

$$
\bar{b}_{q}=\sum_{q^{\prime} \geq q-N_{0}} 2^{\frac{q-q^{\prime}}{2}} b_{q^{\prime}} \in \ell_{q}^{1} .
$$

Vu que $\left\|u_{N}\right\|_{L_{T}^{\infty}\left(\mathcal{B}^{0,1 / 2}\right)} \leq\left\|v_{0}\right\|_{\mathcal{B}^{0,1 / 2}}$, nous obtenons

$$
\left\|\triangle_{q}^{\mathrm{v}}\left(w_{N} \cdot \nabla u_{N}\right)\right\|_{L_{T}^{\infty}\left(L^{2}\right)} \leq 2^{-q / 2} b_{q} C\left(\left\|v_{0}\right\|_{\left.\mathcal{B}^{0,1 / 2}\right)} 2^{5 N / 2}\left\|w_{N}\right\|_{\widetilde{L_{T}^{\infty}}\left(\mathcal{B}^{0,1 / 2}\right)}\right.
$$

et par conséquent, on obtient

$\int_{0}^{T}\left|\left(\triangle_{q}^{\mathrm{v}}\left(w_{N} \cdot \nabla u_{N}\right) \mid \triangle_{q}^{\mathrm{v}} w_{N}\right)_{L^{2}}\right| d \tau \leq 2^{-q} a_{q} C\left(\left\|v_{0}\right\|_{\mathcal{B}^{0,1 / 2}}\right) 2^{5 N / 2} T\left\|w_{N}\right\|_{\widetilde{L_{T}^{\infty}}\left(\mathcal{B}^{0,1 / 2}\right)}^{2}$ avec $a_{q} \in l_{q}^{1 / 2}$. 
Pour le dernier terme nous utilisons

$$
\begin{aligned}
\int_{0}^{T} \mid\left(\triangle_{q}^{\mathrm{v}}\left(u_{N} \nabla u_{N}\right)\left(t^{\prime}\right) \mid\right. & \left.\triangle_{q}^{\mathrm{v}} w_{N}\left(t^{\prime}\right)\right)_{L^{2}} \mid d t^{\prime} \leq \\
& \leq\left\|\triangle_{q}^{\mathrm{v}}\left(\operatorname{div}\left(u_{N} \otimes u_{N}\right)\right)\right\|_{L_{T}^{1}\left(L^{2}\right)}\left\|\triangle_{q}^{\mathrm{v}} w_{N}\right\|_{L_{T}^{\infty}\left(L^{2}\right)} \\
& \leq 2^{N} T\left\|\triangle_{q}^{\mathrm{v}}\left(u_{N} \otimes u_{N}\right)\right\|_{L_{T}^{\infty}\left(L^{2}\right)}\left\|\triangle_{q}^{\mathrm{v}} w_{N}\right\|_{L_{T}^{\infty}\left(L^{2}\right)}
\end{aligned}
$$

À l'aide de la décomposition de Bony, nous obtenons

$$
\begin{aligned}
\left\|\triangle_{q}^{\mathrm{v}}\left(u_{N} \otimes u_{N}\right)\right\|_{L_{T}^{\infty}\left(L^{2}\right)} & \leq 2^{-q / 2} b_{q}\left\|u_{N}\right\|_{L_{T}^{\infty}\left(L^{\infty}\right)}\left\|u_{N}\right\|_{\widetilde{L_{T}^{\infty}}\left(\mathcal{B}^{0,1 / 2}\right)} \\
& \leq 2^{-q / 2} b_{q} 2^{3 N / 2} C\left(\left\|v_{0}\right\|_{\mathcal{B}^{0,1 / 2}}\right)
\end{aligned}
$$

avec $b_{q} \in \ell_{q}^{1}$. Nous obtenons ainsi

$$
\begin{aligned}
\int_{0}^{T} \mid\left(\triangle_{q}^{\mathrm{v}}\left(u_{N} \nabla u_{N}\right)\left(t^{\prime}\right) \mid\right. & \left.\triangle_{q}^{\mathrm{v}} w_{N}\left(t^{\prime}\right)\right)_{L^{2}} \mid d t^{\prime} \leq \\
& \leq 2^{-q} a_{q} C\left(\left\|v_{0}\right\|_{\mathcal{B}^{0,1 / 2}}\right) 2^{5 N / 2} T\left\|w_{N}\right\|_{\widetilde{L_{T}^{\infty}\left(\mathcal{B}^{0,1 / 2}\right)}} .
\end{aligned}
$$

Comme dans la première partie de la démonstration, nous recollons les estimations en obtenant :

$$
\begin{aligned}
& \left\|w_{N}\right\|_{\widetilde{L_{T}^{\infty}}\left(\mathcal{B}^{0,1 / 2}\right)}^{2}+\nu_{h}\left\|\nabla_{h} w_{N}\right\|_{\widetilde{L_{T}^{2}\left(\mathcal{B}^{0,1 / 2}\right)}}^{2} \leq \\
& \leq 4\left\|w_{N}(0)\right\|_{\mathcal{B}^{0,1 / 2}}^{2}+C T 2^{5 N / 2}+C\left\|\nabla_{h} w_{N}\right\|_{\widetilde{L_{T}^{2}}\left(\mathcal{B}^{0,1 / 2}\right)}^{2}\left\|w_{N}\right\|_{\widetilde{L_{T}^{\infty}}\left(\mathcal{B}^{0,1 / 2}\right)} \\
& \quad+T\left(1+2^{2 N}+2^{5 N / 2}\right) C\left(\nu_{h},\left\|v_{0}\right\|_{\left.\mathcal{B}^{0,1 / 2}\right)\left\|w_{N}\right\|_{\widetilde{L_{T}^{\infty}}\left(\mathcal{B}^{0,1 / 2}\right)}^{2}}^{2}\right.
\end{aligned}
$$

Soit maintenant $N$ suffisamment grand, tel que

$$
\left\|w_{N}(0)\right\|_{\mathcal{B}^{0,1 / 2}}=\left\|\left(I-S_{N}\right) v_{0}\right\|_{\mathcal{B}^{0,1 / 2}}<c \nu_{h}
$$

avec $c$ assez petit. Soit ensuite $T$ assez petit, tel que $2^{5 N / 2} T \leq 1$ et tel que $\left\|w_{N}\right\|_{\overparen{L_{T}^{\infty}}\left(\mathcal{B}^{0,1 / 2}\right)} \leq c \nu_{h}$. Alors, nous obtenons directement de l'inégalité (4.3) :

$$
\left\|w_{N}\right\|_{\widetilde{L_{T}^{\infty}\left(\mathcal{B}^{0,1 / 2}\right)}} \leq C\left\|w_{N}(0)\right\|_{\mathcal{B}^{0,1 / 2}} \leq \frac{c \nu_{h}}{2}
$$

pour $2^{5 N / 2} T$ assez petit. On a obtenu ainsi que $w_{N}$ existe localement en temps, donc $v$ existe localement en temps.

Remarque 4.3 Notons que le temps de vie de la solution n'est pas une fonction de $\left\|v_{0}\right\|_{\mathcal{B}^{0,1 / 2}}$ mais dépend de la répartition en fréquences de $v_{0}$. 
Remarque 4.4 Le temps maximal de vie des solutions de $\left(N S_{\nu_{\mathrm{v}}=0}\right)$ dans $\mathcal{B}^{0,1 / 2}$, peut être uniformément minoré lorsque la condition initiale $v_{0}$ varie dans un compacte de $\mathcal{B}^{0,1 / 2}$.

En admetant l'unicité (qu'on va démontrer plus tard) on peut parler du temps maximal de vie de la solution. On a la proposition suivante.

Proposition 2 Soit $T^{*}$ le temps de vie maximal pour la solution $v$ de l'équation $\left(N S_{\nu_{\mathrm{v}}=0}\right)$. Alors, si $T^{*}$ est finie on a $\|v\|_{\widetilde{L_{T^{*}}^{\infty}}\left(\mathcal{B}^{0,1 / 2}\right)}=+\infty$.

Démonstration. Supposons qu'on a $T^{*}$ est fini et $\|v\|_{\widetilde{L_{T^{*}}}\left(\mathcal{B}^{0,1 / 2}\right)}<+\infty$. On va démontrer que si $v$ est définie sur $[0, T)$ avec $\|v\|_{L_{T}^{\infty}\left(\mathcal{B}^{0,1 / 2}\right)}<+\infty$ alors $T$ n'est pas maximal. Comme conséquence de la proposition 1 , nous avons qu'il existe une unique limite de $v\left(t_{n}\right)$ dans $\mathcal{B}^{0,1 / 2}$ lorsque $t_{n}$ tend vers $T^{*}$. On résoud ainsi localement l'équation $\left(N S_{\nu_{\mathrm{v}}=0}\right)$ avec la donnée initiale $v\left(T^{*}\right):=\lim _{t \rightarrow T^{*}} v(t)$. On peut ainsi prolonger la solution sur un intervalle $\left[0, T^{*}+\varepsilon\right)$ ce qui contredit le fait que le temps de vie maximal est fini.

\section{Unicité}

Avant de commencer la démonstration de l'unicité, qui est une application du lemme 4.2, donnons d'abord quelques commentaires sur les difficultés qu'on rencontre et sur les méthodes utilisées pour obtenir le lemme 4.2.

Soient $u$ et $v$ deux fonctions de $L_{T}^{\infty}\left(\mathcal{B}^{0,1 / 2}\right)$ telles que $\nabla_{h} u$ et $\nabla_{h} v$ appartiennent à $L_{T}^{2}\left(\mathcal{B}^{0,1 / 2}\right)$. On suppose que $u$ et $v$ sont solutions de l'équation de $\left(N S_{\nu_{\mathrm{v}}=0}\right)$ pour la même donnée initiale dans $\mathcal{B}^{0,1 / 2}$. On note $w=u-v$ et l'objectif est de démontrer que $w=0$ dans $\mathcal{D}^{\prime}\left(\mathbb{R}_{+}^{*} \times \mathbb{R}^{3}\right)$. L'équation vérifiée par $w$ donne comme termes non-linéaires $v \cdot \nabla w$ et $w \cdot \nabla u$. Les termes $v_{h} \cdot \nabla_{h} w$ et $w_{h} \cdot \nabla_{h} u$ sont estimables à l'aide des lois de produits (ce qui revient en fait à la décomposition de Bony dans la variable verticale). Le terme $v_{3} \cdot \partial_{3} w$ sera estimé dans la suite grâce aux méthodes introduites dans [6] et reprises par exemple dans la démonstration du lemme 4.1.

Reste à estimer le terme $w_{3} \cdot \partial_{3} u$. Vu la régularité dans la troisième variable, il est le produit entre un élément de $B_{2,1}^{1 / 2}$ et un élément de $B_{2,1}^{-1 / 2}$, ce qui donne un élément de $B_{2, \infty}^{-1 / 2}$ (voir la définition 6.1). Cela conduira à faire les estimations sur $w$ dans des espaces qui sont $B_{2, \infty}^{-1 / 2}$ dans la troisième variable (voir aussi [13]). Plus précisement, l'espace candidat pour faire les estimations sur $w$ est $\mathcal{B}_{2, \infty}^{0,-1 / 2}$, avec la norme naturelle

$$
\|w\|_{\mathcal{B}_{2, \infty}^{0,-1 / 2}}=\sup _{q}\left(2^{-\frac{q}{2}}\left\|\triangle_{q}^{\mathrm{v}} w\right\|_{L^{2}}\right) .
$$


Lorsqu'on fait les estimations d'énergie dans l'espace $\mathcal{B}_{2, \infty}^{0,-1 / 2}$ sur l'équation vérifiée par $w$, on va avoir à estimer le terme

$$
\int \triangle_{q}^{\mathrm{v}}\left(w^{3} \partial_{3} u\right) \triangle_{q}^{\mathrm{v}} w d x
$$

La décomposition de Bony dans la variable verticale de ce terme, contient le terme

$$
\int S_{q-1}^{\mathrm{v}} w_{3} \partial_{3} \triangle_{q}^{\mathrm{v}} u \triangle_{q}^{\mathrm{v}} w d x
$$

qui est le plus difficile à estimer.

En effet, lorsqu'on essaie maintenant d'estimer ce terme quand $w$ est dans l'espace $L_{T}^{\infty}\left(\mathcal{B}_{2, \infty}^{0,-1 / 2}\right)$ et $\nabla_{h} w$ dans $L_{T}^{2}\left(\mathcal{B}_{2, \infty}^{0,-1 / 2}\right)$, on obtient

$$
\left|\left(S_{q-1}^{\mathrm{v}} w_{3} \cdot \partial_{3} \triangle_{q}^{\mathrm{v}} u \mid \triangle_{q}^{\mathrm{v}} w\right)_{L^{2}}\right| \leq C\left\|S_{q-1}^{\mathrm{v}} w_{3}\right\|_{L_{\mathrm{v}}^{\infty} L_{h}^{2}}\left\|\triangle_{q}^{\mathrm{v}} \partial_{3} u\right\|_{L_{\mathrm{v}}^{2} L_{h}^{4}}\left\|\triangle_{q}^{\mathrm{v}} w\right\|_{L_{\mathrm{v}}^{2} L_{h}^{4}}
$$

En tenant compte de l'estimation

$$
\begin{aligned}
\left\|\partial_{3} \triangle_{q}^{\mathrm{v}} u\right\|_{L_{\mathrm{v}}^{2} L_{h}^{4}} \leq C 2^{q}\left\|\triangle_{q}^{\mathrm{v}} u\right\|_{L_{\mathrm{v}}^{2} L_{h}^{4}} & \leq C 2^{q}\left\|\triangle_{q}^{\mathrm{v}} u\right\|_{L^{2}}^{1 / 2}\left\|\triangle_{q}^{\mathrm{v}} \nabla_{h} u\right\|_{L^{2}}^{1 / 2} \\
& \leq C 2^{q / 2} b_{q}\|u\|_{\mathcal{B}^{0,1 / 2}}^{1 / 2}\left\|\nabla_{h} u\right\|_{\mathcal{B}^{0,1 / 2}}^{1 / 2}
\end{aligned}
$$

et du fait que

$$
\left\|\triangle_{q}^{\mathrm{v}} w\right\|_{L_{\mathrm{v}}^{2} L_{h}^{4}} \leq\left\|\triangle_{q}^{\mathrm{v}} w\right\|_{L^{2}}^{1 / 2}\left\|\triangle_{q}^{\mathrm{v}} \nabla_{h} w\right\|_{L^{2}}^{1 / 2} \leq C 2^{q / 2}\|w\|_{\mathcal{B}_{2, \infty}^{0,-1 / 2}}^{1 / 2}\left\|\nabla_{h} w\right\|_{\mathcal{B}_{2, \infty}^{0,-1 / 2}}^{1 / 2}
$$

on obtient

$$
\left|\left(S_{q-1}^{\mathrm{v}} w_{3} \cdot \partial_{3} \triangle_{q}^{\mathrm{v}} u \mid \triangle_{q}^{\mathrm{v}} w\right)_{L^{2}}\right| \leq C 2^{q} b_{q}\left\|S_{q-1}^{\mathrm{v}} w_{3}\right\|_{L_{\mathrm{v}}^{\infty} L_{h}^{2}}\|u\|_{\mathcal{B}^{0,1 / 2}}^{1 / 2}\left\|\nabla_{h} u\right\|_{\mathcal{B}^{0,1 / 2}}^{1 / 2}
$$

$$
\times\|w\|_{\mathcal{B}_{2, \infty}^{0,-1 / 2}}^{1 / 2}\left\|\nabla_{h} w\right\|_{\mathcal{B}_{2, \infty}^{0,-1 / 2}}^{1 / 2}
$$

avec $b_{q} \in \ell_{q}^{1}$. Observons maintenant que tant qu'on travaille avec $u$ et $v$ dans la version non-homogène de l'espace $\mathcal{B}^{0,1 / 2}$, on a

$$
\begin{aligned}
\left\|S_{q-1} w_{3}\right\|_{L_{\mathrm{v}}^{\infty} L_{h}^{2}} & \leq C \sum_{-1 \leq q^{\prime} \leq q} 2^{q^{\prime} / 2}\left\|\triangle_{q^{\prime}}^{\mathrm{v}} w_{3}\right\|_{L^{2}} \\
& \leq C\left(\left\|S_{0}^{\mathrm{v}} w^{3}\right\|_{L^{2}}+\sum_{0 \leq q^{\prime} \leq q} 2^{-q^{\prime} / 2}\left\|\triangle_{q^{\prime}}^{\mathrm{v}} \partial_{3} w_{3}\right\|_{L^{2}}\right) .
\end{aligned}
$$

En tenant compte du fait que $\partial_{3} w^{3}=-\operatorname{div}_{h} w^{h}$, on obtient

$$
\left\|\left(S_{q-1}^{\mathrm{v}}-S_{0}^{\mathrm{v}}\right) w_{3}\right\|_{L_{\mathrm{v}}^{\infty} L_{h}^{2}} \leq C q\left\|\nabla_{h} w\right\|_{\mathcal{B}_{2, \infty}^{0,-1 / 2}}
$$


Pour pouvoir utiliser cette inégalité, on introduit un paramètre $N$ et on estime différemment les "fréquences" plus petites que $N$ de celles qui sont plus grandes que $N$. Pour les fréquences qui sont de taille inférieure au paramètre introduit, on utilise la régularité minimale sur $w$, cela veut dire qu'on va tenir compte du fait qu'on estime $w$ dans l'espace $\mathcal{B}_{2, \infty}^{0,-1 / 2}$. Pour les fréquences de taille supérieure au paramètre $N$, on utilise la régularité maximale dont on dispose sur $w$, cela veut dire qu'on tient compte du fait que $w=u-v$ appartient à l'espace $\mathcal{B}^{0,1 / 2}$ (voir aussi [20] pour cette méthode). L'estimation sur les "hautes fréquences" conduit de manière "standard" au choix de $N$ égal à $\left(-\ln \|w\|_{\mathcal{B}_{2, \infty}^{0,-1 / 2}}\right)$. Le problème est d'estimer les "basses fréquences". Le terme qui pose des problèmes est donc

$$
\sum_{0 \leq q \leq N} 2^{-q} \int\left(S_{q-1}^{\mathrm{v}}-S_{0}^{\mathrm{v}}\right) w^{3} \partial_{3} \triangle_{q}^{\mathrm{v}} u \triangle_{q}^{\mathrm{v}} w d x .
$$

Remarquons que les inégalités (4.4) et (4.5), ainsi que la majoration

$$
a b \leq \frac{1}{4} a^{4}+\frac{3}{4} b^{4 / 3}
$$

nous conduisent à l'estimation suivante

$$
\begin{aligned}
\sum_{q \leq N} 2^{-q} & \int\left(S_{q-1}^{\mathrm{v}}-S_{0}^{\mathrm{v}}\right) w^{3} \cdot \partial_{3} \triangle_{q}^{\mathrm{v}} u \triangle_{q}^{\mathrm{v}} w d x \\
& \leq C N\|u\|_{\mathcal{B}^{0,1 / 2}}^{1 / 2}\left\|\nabla_{h} u\right\|_{\mathcal{B}^{0,1 / 2}}^{1 / 2}\|w\|_{\mathcal{B}_{2, \infty}^{0,-1 / 2}}^{1 / 2}\left\|\nabla_{h} w\right\|_{\mathcal{B}_{2, \infty}^{0,-1 / 2}}^{3 / 2} \\
& \leq \frac{C}{\nu_{h}^{3}} N^{4} f(t)\|w\|_{\mathcal{B}_{2, \infty}^{0,-1 / 2}}^{2}+\frac{\nu_{h}}{10}\left\|\nabla_{h} w\right\|_{\mathcal{B}_{2, \infty}^{0,-1 / 2}}^{2},
\end{aligned}
$$

où

$$
f(t)=\|u(t)\|_{\mathcal{B}^{0,1 / 2}}^{2}\left\|\nabla_{h} u(t)\right\|_{\mathcal{B}^{0,1 / 2}}^{2}
$$

est fonction intégrable en temps et où le paramètre $N$ sera choisi à la fin comme étant $N=-\ln \|w\|_{\mathcal{B}_{2, \infty}^{0,-1 / 2}}$. Nous obtenons donc l'inégalité

$$
\frac{d}{d t}\|w(t)\|_{\mathcal{B}_{2, \infty}^{0,-1 / 2}}^{2} \leq C f(t)\|w(t)\|_{\mathcal{B}_{2, \infty}^{0,-1 / 2}}^{2}\left(1-\ln \|w(t)\|_{\mathcal{B}_{2, \infty}^{0,-1 / 2}}^{2}\right)^{4}
$$

où $f(t)$ est une fonction intégrable.

La perte dans cette inégalité est trop grande et ne nous permet pas de déduire l'unicité. Cette difficulté nous oblige de faire les estimations dans l'espace de Sobolev non-homogène $H^{0,-1 / 2}$, afin d'abaisser la perte dans les 
estimations. Cette fois ci, on a les inégalités suivantes

$$
\begin{aligned}
\left\|S_{q-1}^{\mathrm{v}} w_{3}\right\|_{L_{\mathrm{v}}^{\infty} L_{h}^{2}} & \leq C \sum_{-1 \leq q^{\prime} \leq q} 2^{q^{\prime} / 2}\left\|\triangle_{q^{\prime}}^{\mathrm{v}} w^{3}\right\|_{L^{2}} \\
& \leq C\left(\left\|S_{0}^{\mathrm{v}} w_{3}\right\|_{L^{2}}+\sum_{0 \leq q^{\prime} \leq q} 2^{-q^{\prime} / 2}\left\|\triangle_{q^{\prime}}^{\mathrm{v}} \partial_{3} w_{3}\right\|_{L^{2}}\right) \\
& \leq c\left(\sqrt{q}\left\|\nabla_{h} w\right\|_{H^{0,-1 / 2}}+\|w\|_{H^{0,-1 / 2}}\right) .
\end{aligned}
$$

Cette perte dans l'estimation nous amène à utiliser la version paradifférentielle du théorème d'unicité d'Osgood (voir par exemple [20]). Notons toutefois que le terme le plus délicat pose encore des problèmes. En effet, en utilisant l'inégalité (4.7), on obtient l'estimation suivante

$$
\begin{aligned}
\sum_{q \leq N} 2^{-q} & \int\left(S_{q-1}^{\mathrm{v}}-S_{0}^{\mathrm{v}}\right) w^{3} \cdot \partial_{3} \triangle_{q}^{\mathrm{v}} u \triangle_{q}^{\mathrm{v}} w d x \\
& \leq C \sqrt{N}\|u\|_{\mathcal{B}^{0,1 / 2}}^{1 / 2}\left\|\nabla_{h} u\right\|_{\mathcal{B}^{0,1 / 2}}^{1 / 2}\|w\|_{H^{0,-1 / 2}}^{1 / 2}\left\|\nabla_{h} w\right\|_{H^{0,-1 / 2}}^{3 / 2} \\
& \leq \frac{C}{\nu_{h}^{3}} N^{2} f(t)\|w\|_{H^{0,-1 / 2}}^{2}+\frac{\nu_{h}}{10}\left\|\nabla_{h} w\right\|_{H^{0,-1 / 2}}^{2} .
\end{aligned}
$$

où $f(t)$ est fonction intégrable en temps et où le paramètre $N$ sera choisi égal à $\left(-\ln \|w\|_{H^{0,-1 / 2}}\right)$. Ceci conduit à l'inégalité

$$
\frac{d}{d t}\|w(t)\|_{H^{0,-1 / 2}}^{2} \leq C f(t)\|w(t)\|_{H^{0,-1 / 2}}^{2}\left(1-\ln \|w(t)\|_{H^{0,-1 / 2}}\right)^{2}
$$

et cette estimation n'implique toujours pas l'unicité. Cela provient du fait que le problème est critique en variable verticale parce qu'on a à faire des lois des produits entre des espaces avec la somme des indices égale à zéro, mais est également critique dans la variable horizontale parce que la régularité maximale en variable horizontale sur les fonctions utilisées est $\dot{H}^{1}\left(\mathbb{R}_{h}^{2}\right)$, espace qui n'est pas inclus dans $L^{\infty}\left(\mathbb{R}_{h}^{2}\right)$. Afin d'obtenir une estimation optimale, on introduit un nouveau paramètre $\varepsilon$ qui va quantifier le nombre des dérivées dans la variable horizontale utilisées. Le terme

$$
\sum_{0 \leq q \leq N} \int\left(S_{q-1}^{\mathrm{v}}-S_{0}^{\mathrm{v}}\right) w^{3} \partial_{3} \triangle_{q}^{\mathrm{v}} u \triangle_{q}^{\mathrm{v}} w d x
$$

sera estimé maintenant de la manière qui suit. On utilise toujours l'inégalité (4.7), sous la forme

$$
\left\|\left(S_{q-1}^{\mathrm{v}}-S_{0}^{\mathrm{v}}\right) w^{3}\right\|_{L_{\mathrm{v}}^{\infty} L_{h}^{2}} \leq C \sqrt{q}\left\|\nabla_{h} w\right\|_{H^{0,-1 / 2}} .
$$

Afin d'utiliser le moins de dérivées possibles dans la variable horizontale, on utilise le lemme suivant qui est une conséquence des résultats démontrés dans [7]. 
Lemme 4.3 Il existe une constante $C$, telle qu'on a l'inégalité suivante

$$
\|f\|_{L^{2 p}\left(\mathbb{R}_{h}^{2}\right)} \leq C \sqrt{p}\|f\|_{L^{2}\left(\mathbb{R}_{h}^{2}\right)}^{1 / p}\left\|\nabla_{h} f\right\|_{L^{2}\left(\mathbb{R}_{h}^{2}\right)}^{1-1 / p} \text { pour tout } 1 \leq p<+\infty .
$$

Démonstration. Par les inclusions de type Sobolev démontrées dans [7] on a

$$
\|f\|_{L^{2 p}\left(\mathbb{R}_{h}^{2}\right)} \leq C \sqrt{p}\|f\|_{\dot{H}^{1-1 / p}\left(\mathbb{R}_{h}^{2}\right)} .
$$

D'autre part, l'espace $\dot{H}^{1-1 / p}\left(\mathbb{R}_{h}^{2}\right)$ peut s'écrire comme espace d'interpolation entre $L^{2}\left(\mathbb{R}_{h}^{2}\right)$ et $\dot{H}^{1}\left(\mathbb{R}_{h}^{2}\right)$. On obtient ainsi

$$
\|f\|_{\dot{H}^{1-1 / p}\left(\mathbb{R}_{h}^{2}\right)} \leq\|f\|_{L^{2}\left(\mathbb{R}_{h}^{2}\right)}^{1 / p}\left\|\nabla_{h} f\right\|_{L^{2}\left(\mathbb{R}_{h}^{2}\right)}^{1-1 / p} .
$$

On obtient alors

$$
\|f\|_{L^{2 p}\left(\mathbb{R}_{h}^{2}\right)} \leq C \sqrt{p}\|f\|_{L^{2}\left(\mathbb{R}_{h}^{2}\right)}^{1 / p}\left\|\nabla_{h} f\right\|_{L^{2}\left(\mathbb{R}_{h}^{2}\right)}^{1-1 / p} .
$$

Revenons maintenant à l'estimation (4.8) afin de l'améliorer. L'inégalité de Hölder dans la variable horizontale, nous donne

$$
\begin{aligned}
& \sum_{0 \leq q \leq N} 2^{-q} \int\left(S_{q-1}^{\mathrm{v}}-S_{0}^{\mathrm{v}}\right) w^{3} \cdot \partial_{3} \triangle_{q}^{\mathrm{v}} u \triangle_{q}^{\mathrm{v}} w d x \\
& \quad \leq \sum_{0 \leq q \leq N} 2^{-q}\left\|\left(S_{q-1}^{\mathrm{v}}-S_{0}^{\mathrm{v}}\right) w^{3}\right\|_{L_{\mathrm{v}}^{\infty} L_{h}^{2}}\left\|\partial_{3} \triangle_{q}^{\mathrm{v}} u\right\|_{L_{\mathrm{v}}^{2} L_{h}^{\frac{2}{\varepsilon}}}\left\|\triangle_{q}^{\mathrm{v}} w\right\|_{L_{\mathrm{v}}^{2} L_{h}^{1-\varepsilon}} \\
& \quad \leq \sum_{0 \leq q \leq N} C 2^{-q} \sqrt{q}\left\|\nabla_{h} w\right\|_{H^{0,-1 / 2}}\left\|\partial_{3} \triangle_{q}^{\mathrm{v}} u\right\|_{L_{\mathrm{v}}^{2} L_{h}^{\frac{2}{\varepsilon}}\left\|\triangle_{q}^{\mathrm{v}} w\right\|_{L_{\mathrm{v}}^{2} L_{h}^{\frac{2}{1-\varepsilon}}} .}
\end{aligned}
$$

En utilisant maintenant le lemme 4.3 on obtient pour $0<\varepsilon<1 / 2$

$$
\begin{gathered}
\sum_{0 \leq q \leq N} 2^{-q} \int\left(S_{q-1}^{\mathrm{v}}-S_{0}^{\mathrm{v}}\right) w^{3} \partial_{3} \triangle_{q}^{\mathrm{v}} u \triangle_{q}^{\mathrm{v}} w d x \leq \sum_{0 \leq q \leq N} C 2^{-q} \sqrt{\frac{q}{\varepsilon}}\left\|\nabla_{h} w\right\|_{H^{0,-1 / 2}} \times \\
\times\left\|\triangle_{q}^{\mathrm{v}} \partial_{3} u\right\|_{L^{2}}^{\varepsilon}\left\|\triangle_{q}^{\mathrm{v}} \nabla_{h} \partial_{3} u\right\|_{L^{2}}^{1-\varepsilon}\left\|\triangle_{q}^{\mathrm{v}} w\right\|_{L^{2}}^{1-\varepsilon}\left\|\nabla_{h} \triangle_{q}^{\mathrm{v}} w\right\|_{L^{2}}^{\varepsilon} .
\end{gathered}
$$

Vu qu'on a

$$
\left\|\triangle_{q}^{\mathrm{v}} \partial_{3} u\right\|_{L^{2}}^{\varepsilon}\left\|\nabla_{h} \triangle_{q}^{\mathrm{v}} \partial_{3} u\right\|_{L^{2}}^{1-\varepsilon} \leq 2^{q} C\left\|\triangle_{q}^{\mathrm{v}} u\right\|_{L^{2}}^{\varepsilon}\left\|\nabla_{h} \triangle_{q}^{\mathrm{v}} u\right\|_{L^{2}}^{1-\varepsilon}
$$

et en utilisant les estimations

$$
\left\|\triangle_{q}^{\mathrm{v}} u\right\|_{L^{2}}^{\varepsilon}\left\|\nabla_{h} \triangle_{q}^{\mathrm{v}} u\right\|_{L^{2}}^{1-\varepsilon} \leq 2^{-q / 2} b_{q}\|u\|_{\mathcal{B}^{0,1 / 2}}^{\varepsilon}\left\|\nabla_{h} u\right\|_{\mathcal{B}^{0,1 / 2}}^{1-\varepsilon}
$$

et

$$
\left\|\triangle_{q}^{\mathrm{v}} w\right\|_{L^{2}}^{1-\varepsilon}\left\|\nabla_{h} \triangle_{q}^{\mathrm{v}} w\right\|_{L^{2}}^{\varepsilon} \leq 2^{q / 2} c_{q}\|w\|_{H^{0,-1 / 2}}^{1-\varepsilon}\left\|\nabla_{h} w\right\|_{H^{0,-1 / 2}}^{\varepsilon},
$$


on obtient

$$
\begin{aligned}
& \sum_{0 \leq q \leq N} 2^{-q} \int\left(S_{q-1}^{\mathrm{v}}-S_{0}^{\mathrm{v}}\right) w^{3} \partial_{3} \triangle_{q}^{\mathrm{v}} u \triangle_{q}^{\mathrm{v}} w d x \leq \\
& \leq C \sqrt{\frac{N}{\varepsilon}}\|u\|_{\mathcal{B}^{0,1 / 2}}^{\varepsilon}\left\|\nabla_{h} u\right\|_{\mathcal{B}^{0,1 / 2}}^{1-\varepsilon}\|w\|_{H^{0,-1 / 2}}^{1-\varepsilon}\left\|\nabla_{h} w\right\|_{H^{0,-1 / 2}}^{1+\varepsilon} .
\end{aligned}
$$

En utilisant maintenant l'inégalité

$$
a b \leq a^{\frac{2}{1+\varepsilon}}+b^{\frac{2}{1-\varepsilon}}
$$

on obtient

$$
\begin{aligned}
& \sum_{0 \leq q \leq N} 2^{-q} \int\left(S_{q-1}^{\mathrm{v}}-S_{0}^{\mathrm{v}}\right) w^{3} \partial_{3} \triangle_{q}^{\mathrm{v}} u \triangle_{q}^{\mathrm{v}} w d x \\
& \leq\left(\frac{C^{2}}{\nu_{h}^{1+\varepsilon}}\right)^{\frac{1}{1-\varepsilon}}\left(\frac{N}{\varepsilon}\right)^{\frac{1}{1-\varepsilon}}\|u\|_{\mathcal{B}^{0,1 / 2}}^{\frac{2 \varepsilon}{1-\varepsilon}}\left\|\nabla_{h} u\right\|_{\mathcal{B}^{0,1 / 2}}^{2}\|w\|_{H^{0,-1 / 2}}^{2}+\frac{\nu_{h}}{2}\left\|\nabla_{h} w\right\|_{H^{0,-1 / 2}}^{2} .
\end{aligned}
$$

Puisque $\varepsilon$ est un petit paramètre, on peut considerer $0<\varepsilon<1 / 2$ et on a

$$
\left(\frac{C^{2}}{\nu_{h}^{1+\varepsilon}}\right)^{\frac{1}{1-\varepsilon}} \leq \frac{\max \left\{1, C^{4}\right\}}{\min \left\{1, \nu_{h}\right\}^{3}} .
$$

Dans toute la suite, on va désigner cette constante par $C$.

Pour optimiser l'estimation, on fait le choix de $\varepsilon$ égal à $\frac{1}{1+\ln N}$ et on obtient

$$
\begin{aligned}
& \sum_{0 \leq q \leq N} 2^{-q} \int\left(S_{q-1}^{\mathrm{v}}-S_{0}^{\mathrm{v}}\right) w^{3} \partial_{3}^{\mathrm{v}} \triangle_{q}^{\mathrm{v}} u \triangle_{q}^{\mathrm{v}} w d x \leq \\
& \quad \leq C N(1+\ln N) f(t)\|w\|_{H^{0,-1 / 2}}^{2}+\frac{\nu_{h}}{2}\left\|\nabla_{h} w\right\|_{H^{0,-1 / 2}}^{2} .
\end{aligned}
$$

Le choix de $N=-\ln \|w\|_{H^{0,-1 / 2}}^{2}$, nous amène à l'inégalité différentielle

$$
\begin{aligned}
& \frac{d}{d t}\|w(t)\|_{H^{0,-1 / 2}}^{2} \leq \\
& \quad \leq C\|w(t)\|_{H^{0,-1 / 2}}^{2}\left(-\ln \|w(t)\|_{H^{0,-1 / 2}}^{2}\right) \ln \left(-\ln \|w(t)\|_{H^{0,-1 / 2}}^{2}\right),
\end{aligned}
$$

ce qui nous donne l'unicité à l'aide du lemme d'Osgood.

La démonstration rigoureuse de l'estimation (4.9) est donnée dans la section 5.2. 
Nous allons démontrer l'unicité dans l'espace $H^{0,1 / 2}$, la preuve dans $\mathcal{B}^{0,1 / 2}$ étant plus simple vu qu'on a $\left\|S_{q}^{\mathrm{v}} u\right\|_{L_{\mathrm{v}}^{\infty}\left(L_{h}^{2}\right)} \leq C\|u\|_{\mathcal{B}^{0,1 / 2}}$, or dans l'espace $H^{0,1 / 2}$ on a à eviter aussi la difficulté

$$
\left\|S_{q}^{\mathrm{v}} u\right\|_{L_{\mathrm{v}}^{\infty}\left(L_{h}^{2}\right)} \leq C \sqrt{q}\|u\|_{H^{0,1 / 2}}
$$

Remarquons aussi, qu'on ne peut pas travailler dans la version homogène de l'espace $H^{0,1 / 2}$, parce que, dans cet espace $S_{q}^{\mathrm{v}} u$ n'appartient pas nécessairement à $L_{\mathrm{v}}^{\infty} L_{h}^{2}$. Signalons également que pour l'unicité dans l'espace homogène $\mathcal{B}^{0,1 / 2}$, on va démontrer que $w$, la différence entre les deux solutions est nulle dans la norme

$$
\|w\|^{2}:=\left\|S_{0}^{\mathrm{v}} w\right\|_{L_{\mathrm{v}}^{\infty} L_{h}^{2}}^{2}+\sum_{q \geq 0} 2^{-q}\left\|\triangle_{q}^{\mathrm{v}} w\right\|_{L^{2}}^{2}
$$

\section{Unicité pour l'équation de Navier-Stokes anisotrope dans l'es- pace $H^{0,1 / 2}$}

Dans la suite nous allons démontrer l'unicité dans l'espace $H^{0,1 / 2}$. Soient ainsi $u$ et $v$ dans $L_{l o c}^{\infty}\left(H^{0,1 / 2}\right)$ avec $\nabla_{h} u$ et $\nabla_{h} v$ dans $L_{l o c}^{2}\left(H^{0,1 / 2}\right)$, deux solutions pour la même donnée initiale. Nous allons démontrer que $w=0$ dans $L_{T}^{\infty}\left(H^{0,-1 / 2}\right)$ avec $\nabla_{h} w=0$ dans $L_{T}^{2}\left(H^{0,-1 / 2}\right)$. Pour cela on va faire les estimations d'énergie dans $H^{0,-1 / 2}$ sur l'équation vérifiée par $w$. L'équation vérifiée par $w$ est la suivante

$$
\partial_{t} w+v \nabla w+w \nabla u-\nu_{h} \Delta_{h} w=-\nabla p .
$$

Le lemme 4.2 nous donne alors

$$
\begin{aligned}
\frac{d}{d t}\|w(t)\|_{H^{0,-1 / 2}}^{2} \leq C f(t)\|w(t)\|_{H^{0,-1 / 2}}^{2} & \left(1-\ln \|w(t)\|_{H^{0,-1 / 2}}^{2}\right) \times \\
& \times \ln \left(1-\ln \|w(t)\|_{H^{0,-1 / 2}}^{2}\right),
\end{aligned}
$$

où $f(t)$ est une fonction intégrable sur l'intervalle $[0, T]$ avec

$$
\begin{aligned}
\|f\|_{L_{T}^{1}} \leq C & \left(\left\|\nabla_{h} u\right\|_{L_{T}^{2}\left(H^{0,1 / 2}\right)}^{2}+\left\|\nabla_{h} v\right\|_{L_{T}^{2}\left(H^{0,1 / 2}\right)}^{2}+\left\|\nabla_{h} w\right\|_{L_{T}^{2}\left(H^{0,1 / 2}\right)}^{2}\right) \\
& \times\left(\|u\|_{L_{T}^{\infty}\left(H^{0,1 / 2}\right)}^{2}+\|v\|_{L_{T}^{\infty}\left(H^{0,1 / 2}\right)}^{2}+\|w\|_{L_{T}^{\infty}\left(H^{0,1 / 2}\right)}^{2}\right) .
\end{aligned}
$$

En tenant maintenant compte du fait que $w=u-v$, on a

$$
\|w\|_{L_{T}^{\infty}\left(H^{0,1 / 2}\right)} \leq\|u\|_{L_{T}^{\infty}\left(H^{0,1 / 2}\right)}+\|v\|_{L_{T}^{\infty}\left(H^{0,1 / 2}\right)},
$$

et aussi

$$
\left\|\nabla_{h} w\right\|_{L_{T}^{2}\left(H^{0,1 / 2}\right)} \leq\left\|\nabla_{h} u\right\|_{L_{T}^{2}\left(H^{0,1 / 2}\right)}+\left\|\nabla_{h} v\right\|_{L_{T}^{2}\left(H^{0,1 / 2}\right)} .
$$

Rappelons à présent le lemme d'Osgood (voir [8]), qui nous va permettre de conclure à l'unicité. 
Lemme 4.4 (Osgood) Soient $\rho \geq 0$ une fonction mesurable, $\gamma$ une fonction localement intégrable et $\mu$ une fonction continue croissante qui vérifie la condition suivante

$$
\int_{0}^{1} \frac{d r}{\mu(r)}=+\infty
$$

Soient aussi a un nombre réel positif et $\rho$ vérifiant l'inégalité

$$
\rho(t) \leq a+\int_{0}^{t} \gamma(s) \mu(\rho(s)) d s .
$$

Alors, si a est nul la fonction $\rho$ est identiquement nulle. Si a est non nul, alors on a

$$
-\mathcal{M}(\rho(t))+\mathcal{M}(a) \leq \int_{0}^{t} \gamma(s) d s, \quad \text { avec } \quad \mathcal{M}(x)=\int_{x}^{1} \frac{d r}{\mu(r)} .
$$

Finalement, l'application du lemme d'Osgood, dans le cas où on a $\rho(t)=$ $\|w(t)\|_{H^{0,-1 / 2}}^{2}$ et $\mu(r)=r(-\ln r) \ln (-\ln r)$, implique l'unicité.

Pour la démonstration de l'unicité dans le cas de l'espace de Besov homogène $\mathcal{B}^{0,1 / 2}$ on va signaler les différences de démonstration par rapport au cas de l'espace de Sobolev inhomogène $H^{0,1 / 2}$. Nous avons déjà remarqué dans la discussion du début qu'on va estimer $w$ dans la norme suivante

$$
\begin{aligned}
\left\|S_{0}^{\mathrm{v}} w(t)\right\|_{L_{\mathrm{v}}^{\infty} L_{h}^{2}}^{2}+\sum_{q \geq 0} 2^{-q}\left\|\triangle_{q}^{\mathrm{v}} w(t)\right\|_{L^{2}}^{2} & +\nu_{h} \sup _{x_{3}} \int_{0}^{t}\left\|S_{0}^{\mathrm{v}} \nabla_{h} w\left(\tau, \cdot, x_{3}\right)\right\|_{L_{h}^{2}}^{2} d \tau \\
& +\nu_{h} \sum_{q \geq 0} 2^{-q} \int_{0}^{t}\left\|\nabla_{h} \triangle_{q}^{\mathrm{v}} w(\tau)\right\|_{L^{2}}^{2} d \tau .
\end{aligned}
$$

Notons que pour estimer $\left\|S_{0}^{\mathrm{v}} w\right\|_{L_{\mathrm{v}}^{\infty} L_{h}^{2}}$ on fait une estimation d'énergie dans la variable horizontale et on prend le supremum dans la variable verticale. On obtient ainsi

$$
\begin{aligned}
\| S_{0}^{\mathrm{v}} w & \left\|_{L_{\mathrm{v}} L_{h}^{2}}^{2}+\nu_{h} \sup _{x_{3}} \int_{0}^{t}\right\| S_{0}^{\mathrm{v}} \nabla_{h} w\left(x_{3}, \tau\right) \|_{L_{h}^{2}}^{2} d \tau \leq \\
\leq & \sup _{x_{3}} \int_{0}^{t}\left|\left(S_{0}^{\mathrm{v}} \operatorname{div}(u \otimes w)\left(x_{3}, \tau\right) \mid S_{0}^{\mathrm{v}} w\left(x_{3}, \tau\right)\right)_{L_{h}^{2}}\right| d \tau \\
& \quad+\sup _{x_{3}} \int_{0}^{t}\left|\left(S_{0}^{\mathrm{v}} \nabla \sum_{i, j} \partial_{i} \partial_{j} \Delta^{-1} S_{0}^{\mathrm{v}}\left(u_{i} w_{j}\right)\left(x_{3}, \tau\right) \mid S_{0}^{\mathrm{v}} w\left(x_{3}, \tau\right)\right)_{L_{h}^{2}}\right| d \tau .
\end{aligned}
$$

On ne va pas donner les estimations exactes, elles sont très faciles à obtenir $\mathrm{vu}$ qu'on travaille avec des fonctions localisées sur de "basses" fréquences dans la variable verticale. 
Remarque 4.5 Finalement, signalons qu'une autre possibilité est d'estimer $w$ dans la norme suivante

$$
|w|:=\sum_{q \leq 0} 2^{q / 2}\left\|\triangle_{q}^{\mathrm{v}} w\right\|_{L^{2}}+\left(\sum_{q \geq 0} 2^{-q}\left\|\triangle_{q}^{\mathrm{v}} w\right\|_{L^{2}}^{2}\right)^{1 / 2} .
$$

Cela nous montre aussi que l'espace homogène maximal où on peut démontrer l'unicité est l'espace des fonctions u qui ont la propriété que $\left(I-S_{0}^{\mathrm{v}}\right) u$ appartient à $H^{0,1 / 2}$ et $S_{0}^{\mathrm{v}} u$ appartient à $\mathcal{B}^{0,1 / 2}$, avec la norme évidente :

$$
\left\|\left|u\|\mid=\| S_{0}^{\mathrm{v}} u\left\|_{\mathcal{B}^{0,1 / 2}}+\right\|\left(I-S_{0}^{\mathrm{v}}\right) u \|_{H^{0,1 / 2}} .\right.\right.
$$

\section{Démonstrations des estimations d'énergie}

\subsection{L'estimation d'énergie pour l'équation de Navier-Stokes}

Donnons maintenant la démonstration de l'estimation d'énergie anisotrope sur le terme convectif, dans le cadre des espaces de type $\widetilde{L_{T}^{p}}\left(\mathcal{B}^{0,1 / 2}\right)$, énoncée dans le lemme 4.1. Rappelons ici le lemme dont il s'agit.

Lemme (estimation pour l'existence locale dans $\mathbb{R}^{3}$ ). Il existe une constante strictement positive $C$, telle que pour tous les champs de vecteurs $u$ et $v$ dépendant de temps tels que $u(t)$ soit de divergence nulle pour tout $t \in[0, T]$, on $a:$

$$
\begin{aligned}
& \int_{0}^{T}\left|\left(\triangle_{q}^{\mathrm{v}}(u \cdot \nabla v)(\tau) \mid \triangle_{q}^{\mathrm{v}} v(\tau)\right)_{L^{2}}\right| d \tau \leq \\
& \leq C a_{q} 2^{-q}\left(\|u\|_{\overparen{L_{T}^{\infty}}\left(\mathcal{B}^{0, \frac{1}{2}}\right)}^{\frac{1}{2}}\left\|\nabla_{h} u\right\|_{\widetilde{L_{T}^{2}}\left(\mathcal{B}^{0, \frac{1}{2}}\right)}^{\frac{1}{2}}\|v\|_{\widetilde{L_{T}^{\infty}}\left(\mathcal{B}^{0, \frac{1}{2}}\right)}^{\frac{1}{2}}\left\|\nabla_{h} v\right\|_{\widetilde{L_{T}^{2}}\left(\mathcal{B}^{0, \frac{1}{2}}\right)}^{\frac{3}{2}}\right. \\
& \left.\quad+\left\|\nabla_{h} u\right\|_{\widetilde{L_{T}^{2}}\left(\mathcal{B}^{0}, \frac{1}{2}\right)}\|v\|_{\widetilde{L_{T}^{\infty}}\left(\mathcal{B}^{0, \frac{1}{2}}\right)}\left\|\nabla_{h} v\right\|_{\widetilde{L_{T}^{2}}\left(\mathcal{B}^{0, \frac{1}{2}}\right)}\right)
\end{aligned}
$$

avec $a_{q} \geq 0$ et $\sum_{q \in \mathbb{Z}} a_{q}^{1 / 2} \leq 1$.

Démonstration. Nous allons suivre la démonstration donnée dans [6] en travaillant dans le contexte des espaces $\widetilde{L_{T}^{p}}(\mathcal{B})$, et en utilisant de manière convenable l'inégalité de Hölder pour les intégrations en temps. Soit donc

$$
F_{q}^{h}=\triangle_{q}^{\mathrm{v}}\left(u^{h} \cdot \nabla_{h} v\right) \quad \text { et } \quad F_{q}^{\mathrm{v}}=\triangle_{q}^{\mathrm{v}}\left(u_{3} \cdot \partial_{3} v\right) .
$$

Pour estimer $\int_{0}^{T}\left|\left(F_{q}^{h}(\tau) \mid \triangle_{q}^{\mathrm{v}} v(\tau)\right)_{L^{2}}\right| d \tau$, on va utiliser l'inégalité de Hölder dans la variable verticale et horizontale et la décomposition de Bony dans la variable verticale. Nous avons dans la variable verticale:

$$
\left|\left(F_{q}^{h}(\tau) \mid \triangle_{q}^{\mathrm{v}} v(\tau)\right)_{L^{2}\left(\mathbb{R}^{3}\right)}\right| \leq \int\left\|F_{q}^{h}\left(\tau, x_{h}, \cdot\right)\right\|_{L_{\mathrm{v}}^{2}}\left\|\triangle_{q}^{\mathrm{v}} v\left(\tau, x_{h}, \cdot\right)\right\|_{L_{\mathrm{v}}^{2}} d x_{h} .
$$


En utilisant l'inégalité de Hölder dans la variable horizontale, on obtient

$$
\left|\left(F_{q}^{h}(\tau) \mid \triangle_{q}^{\mathrm{v}} v(\tau)\right)_{L^{2}\left(\mathbb{R}^{3}\right)}\right| \leq\left\|F_{q}^{h}(\tau, \cdot, \cdot)\right\|_{L_{h}^{4 / 3}\left(L_{\mathrm{v}}^{2}\right)}\left\|\triangle_{q}^{\mathrm{v}} v(\tau, \cdot, \cdot)\right\|_{L_{h}^{4}\left(L_{\mathrm{v}}^{2}\right)} .
$$

En utilisant l'inégalité (3.7) du lemme 3.3, on obtient

$$
\left|\left(F_{q}^{h}(\tau) \mid \triangle_{q}^{\mathrm{v}} v(\tau)\right)_{L^{2}}\right| \leq\left\|F_{q}^{h}(\tau)\right\|_{L_{h}^{4 / 3}\left(L_{\mathrm{v}}^{2}\right)}\left\|\triangle_{q}^{\mathrm{v}} v(\tau)\right\|_{L^{2}\left(\mathbb{R}^{3}\right)}^{1 / 2}\left\|\triangle_{q}^{\mathrm{v}} \nabla_{h} v(\tau)\right\|_{L^{2}\left(\mathbb{R}^{3}\right)}^{1 / 2} .
$$

L'intégration en temps sur l'intervalle $[0, T]$ et l'inégalité de Hölder en temps, nous donnent

$$
\begin{aligned}
\int_{0}^{T} \mid\left(F_{q}^{h}(\tau)\right. & \left.\mid \triangle_{q}^{\mathrm{v}} v(\tau)\right)_{L^{2}} \mid d \tau \leq \\
& \leq\left\|F_{q}^{h}\right\|_{L_{T}^{4 / 3}\left(L_{h}^{4 / 3} L_{\mathrm{v}}^{2}\right)}\left\|\triangle_{q}^{\mathrm{v}} v\right\|_{L_{T}^{\infty}\left(L^{2}\left(\mathbb{R}^{3}\right)\right)}^{1 / 2}\|\| \triangle_{q}^{\mathrm{v}} \nabla_{h} v(\tau)\left\|_{L^{2}\left(\mathbb{R}^{3}\right)}^{1 / 2}\right\|_{L^{4}([0, T])} \\
& \leq\left\|F_{q}^{h}\right\|_{L_{T}^{4 / 3}\left(L_{h}^{4 / 3} L_{\mathrm{v}}^{2}\right)}\left\|\triangle_{q}^{\mathrm{v}} v\right\|_{L_{T}^{\infty}\left(L^{2}\left(\mathbb{R}^{3}\right)\right)}^{1 / 2}\left\|\triangle_{q}^{\mathrm{v}} \nabla_{h} v(\tau)\right\|_{L_{T}^{2}\left(L^{2}\left(\mathbb{R}^{3}\right)\right)}^{1 / 2}
\end{aligned}
$$

Si on note

$$
b_{q}^{(1)}=\frac{2^{q / 2}\left\|\triangle_{q}^{\mathrm{v}} v\right\|_{L_{T}^{\infty}\left(L^{2}\left(\mathbb{R}^{3}\right)\right)}}{\|v\|_{\widetilde{L_{T}^{\infty}}\left(\mathcal{B}^{0,1 / 2}\right)}} \quad \text { et } \quad b_{q}^{(2)}=\frac{2^{q / 2}\left\|\triangle_{q}^{\mathrm{v}} \nabla_{h} v\right\|_{L_{T}^{2}\left(L^{2}\left(\mathbb{R}^{3}\right)\right)}}{\left\|\nabla_{h} v\right\|_{\widetilde{L_{T}^{2}}\left(\mathcal{B}^{0,1 / 2}\right)}},
$$

alors

$$
\begin{aligned}
\int_{0}^{T} \mid\left(F_{q}^{h}(\tau) \mid\right. & \left.\triangle_{q}^{\mathrm{v}} v(\tau)\right)_{L^{2}} \mid d \tau \leq \\
& \leq 2^{-q / 2} C b_{q}\left\|F_{q}^{h}\right\|_{L_{T}^{4 / 3}\left(L_{h}^{4 / 3} L_{\mathrm{v}}^{2}\right)}\|v\|_{\widetilde{L_{T}^{\infty}\left(\mathcal{B}^{0,1 / 2}\right)}}^{1 / 2}\left\|\nabla_{h} v\right\|_{\widetilde{L_{T}^{2}}\left(\mathcal{B}^{0,1 / 2}\right)}^{1 / 2}
\end{aligned}
$$

avec $b_{q}=\sqrt{b_{q}^{(1)} b_{q}^{(2)}} \in \ell_{q}^{1}$.

Estimons maintenant $\left\|\triangle_{q}^{\mathrm{v}}\left(u^{h} \cdot \nabla_{h} v\right)\right\|_{L_{h}^{4 / 3}\left(L_{\mathrm{v}}^{2}\right)}$. En décomposant en termes de paraproduits et de reste, on a

$$
\begin{aligned}
& \triangle_{q}^{\mathrm{v}}\left(u^{h} \cdot \nabla_{h} v\right)=\sum_{\left|q^{\prime}-q\right| \leq 4} \triangle_{q}^{\mathrm{v}}\left(S_{q^{\prime}-1}^{\mathrm{v}} u^{h} \cdot \triangle_{q^{\prime}}^{\mathrm{v}} \nabla_{h} v\right)+\sum_{\left|q^{\prime}-q\right| \leq 4} \triangle_{q}^{\mathrm{v}}\left(S_{q^{\prime}-1}^{\mathrm{v}} \nabla_{h} v \cdot \triangle_{q^{\prime}}^{\mathrm{v}} u^{h}\right) \\
& \quad+\sum_{\substack{i \in\{0, \pm 1\} \\
q^{\prime}>q-4}} \triangle_{q}^{\mathrm{v}}\left(\triangle_{q^{\prime}}^{\mathrm{v}} u^{h} \cdot \triangle_{q^{\prime}-i}^{\mathrm{v}} \nabla_{h} v\right) .
\end{aligned}
$$

Estimons la première somme, qui est en fait $\triangle_{q}^{\mathrm{v}} T_{u} \nabla_{h} v$. On a

$$
\left\|\triangle_{q}^{\mathrm{v}} T_{u} \nabla_{h} v\left(\tau, x_{h}, \cdot\right)\right\|_{L_{\mathrm{v}}^{2}} \leq \sum_{\left|q^{\prime}-q\right| \leq 4}\left\|S_{q^{\prime}-1}^{\mathrm{v}} u\left(\tau, x_{h}, \cdot\right)\right\|_{L_{\mathrm{v}}^{\infty}}\left\|\triangle_{q^{\prime}}^{\mathrm{v}} \nabla_{h} v\left(\tau, x_{h}, \cdot\right)\right\|_{L_{\mathrm{v}}^{2}}
$$


En prenant la norme $L_{h}^{4 / 3}$ et en appliquant l'inégalité de Hölder, on obtient :

$$
\left\|\triangle_{q}^{\mathrm{v}} T_{u} \nabla_{h} v(\tau)\right\|_{L_{h}^{4 / 3}\left(L_{\mathrm{v}}^{2}\right)} \leq \sum_{\left|q^{\prime}-q\right| \leq 4}\left\|S_{q^{\prime}-1}^{\mathrm{v}} u(\tau)\right\|_{L_{h}^{4}\left(L_{\mathrm{v}}^{\infty}\right)}\left\|\triangle_{q^{\prime}}^{\mathrm{v}} \nabla_{h} v(\tau)\right\|_{L^{2}\left(\mathbb{R}^{3}\right)} .
$$

D'après le corollaire 2 , on a

$$
\left\|\triangle_{q}^{\mathrm{v}} T_{u} \nabla_{h} v(\tau)\right\|_{L_{h}^{4 / 3}\left(L_{\mathrm{V}}^{2}\right)} \leq\|u(\tau)\|_{\mathcal{B}^{0,1 / 2}}^{1 / 2}\left\|\nabla_{h} u(\tau)\right\|_{\mathcal{B}^{0,1 / 2}}^{1 / 2} \sum_{\left|q-q^{\prime}\right| \leq 4}\left\|\triangle_{q^{\prime}}^{\mathrm{v}} \nabla_{h} v(\tau)\right\|_{L^{2}\left(\mathbb{R}^{3}\right)}
$$

En prenant la norme $L_{T}^{4 / 3}$ et en utilisant l'inégalité de Hölder en temps, nous obtenons

$$
\begin{aligned}
& \left\|\triangle_{q}^{\mathrm{v}} T_{u} \nabla_{h} v(\tau)\right\|_{L_{T}^{4 / 3}\left(L_{h}^{4 / 3} L_{\mathrm{v}}^{2}\right)} \leq\|u\|_{L_{T}^{\infty}\left(\mathcal{B}^{0,1 / 2}\right)}^{1 / 2}\left\|\nabla_{h} u\right\|_{L_{T}^{2}\left(\mathcal{B}^{0,1 / 2}\right)}^{1 / 2} \sum_{\left|q-q^{\prime}\right| \leq 4}\left\|\triangle_{q^{\prime}}^{\mathrm{v}} \nabla_{h} v\right\|_{L_{T}^{2}\left(L^{2}\right)} \\
& \quad \leq 2^{-q / 2} C b_{q}^{(2)}\|u\|_{L_{T}^{\infty}\left(\mathcal{B}^{0,1 / 2}\right)}^{1 / 2}\left\|\nabla_{h} u\right\|_{L_{T}^{2}\left(\mathcal{B}^{0,1 / 2}\right)}^{1 / 2}\left\|\nabla_{h} v\right\|_{\widetilde{L_{T}^{2}\left(\mathcal{B}^{0,1 / 2}\right)}}
\end{aligned}
$$

avec

$$
b_{q}^{(2)}=\sum_{\left|q-q^{\prime}\right| \leq 4} \frac{2^{q^{\prime} / 2}\left\|\triangle_{q^{\prime}}^{\mathrm{v}} \nabla_{h} v\right\|_{L_{T}^{2}\left(L^{2}\left(\mathbb{R}^{3}\right)\right)}}{\left\|\nabla_{h} v\right\|_{\widetilde{L_{T}^{2}}\left(\mathcal{B}^{0,1 / 2}\right)}}, \quad b_{q}^{(2)} \in \ell_{q}^{1} .
$$

La deuxième somme de la relation (5.1), qui est en fait $\triangle_{q}^{\mathrm{v}} T_{\nabla_{h} v} u$, s'estime comme suit :

$$
\left\|\triangle_{q}^{\mathrm{v}} T_{\nabla_{h} v} u\right\|_{L_{h}^{4 / 3} L_{\mathrm{v}}^{2}} \leq \sum_{\left|q^{\prime}-q\right| \leq 4}\left\|S_{q^{\prime}-1}^{\mathrm{v}} \nabla_{h} v\right\|_{L_{h}^{2}\left(L_{\mathrm{v}}^{\infty}\right)}\left\|\triangle_{q^{\prime}}^{\mathrm{v}} u\right\|_{L_{h}^{4}\left(L_{\mathrm{v}}^{2}\right)}
$$

En utilisant le corollaire 2 et le lemme 3.3, on obtient

$$
\left\|\triangle_{q}^{\mathrm{v}} T_{\nabla_{h} v} u\right\|_{L_{h}^{4 / 3} L_{\mathrm{v}}^{2}} \leq\left\|\nabla_{h} v\right\|_{\mathcal{B}^{0,1 / 2}} \sum_{\left|q^{\prime}-q\right| \leq 4}\left\|\triangle_{q^{\prime}}^{\mathrm{v}} u\right\|_{L^{2}\left(\mathbb{R}^{3}\right)}^{1 / 2}\left\|\triangle_{q^{\prime}}^{\mathrm{v}} \nabla_{h} u\right\|_{L^{2}\left(\mathbb{R}^{3}\right)}^{1 / 2} .
$$

En prenant la norme $L_{T}^{4 / 3}$ et en utilisant l'inégalité de Hölder, on a

$$
\begin{aligned}
\left\|\triangle_{q}^{\mathrm{v}} T_{\nabla_{h} v} u\right\|_{L_{T}^{4 / 3}\left(L_{h}^{4 / 3} L_{\mathrm{v}}^{2}\right)} & \leq\left\|\nabla_{h} v\right\|_{L_{T}^{2}\left(\mathcal{B}^{0,1 / 2}\right)} \sum_{\left|q^{\prime}-q\right| \leq 4}\left\|\triangle_{q^{\prime}}^{\mathrm{v}} u\right\|_{L_{T}^{\infty}\left(L^{2}\right)}^{1 / 2}\left\|\triangle_{q^{\prime}}^{\mathrm{v}} \nabla_{h} u\right\|_{L_{T}^{2}\left(L^{2}\right)}^{1 / 2} \\
& \leq 2^{-q / 2} C \tilde{b}_{q}\left\|\nabla_{h} v\right\|_{L_{T}^{2}\left(\mathcal{B}^{0,1 / 2}\right)}\|u\|_{L_{T}^{\infty}\left(\mathcal{B}^{0,1 / 2}\right)}^{1 / 2}\left\|\nabla_{h} u\right\|_{\tilde{L}_{T}^{2}\left(\mathcal{B}^{0,1 / 2}\right)}^{1 / 2}
\end{aligned}
$$

$\operatorname{avec} \tilde{b}_{q}=\sqrt{\tilde{b}_{q}^{(1)} \tilde{b}_{q}^{(2)}} \in \ell_{q}^{1}$, où

$$
\tilde{b}_{q}^{(1)}=\frac{2^{q / 2}\left\|\triangle_{q}^{\mathrm{v}} u\right\|_{L_{T}^{\infty}\left(L^{2}\right)}}{\|u\|_{\widetilde{L_{T}^{\infty}}\left(\mathcal{B}^{0,1 / 2}\right)}} \in \ell_{q}^{1} \quad \text { et } \quad \tilde{b}_{q}^{(2)}=\frac{2^{q / 2}\left\|\triangle_{q}^{\mathrm{v}} \nabla_{h} u\right\|_{L_{T}^{2}\left(L^{2}\right)}}{\left\|\nabla_{h} u\right\|_{\widetilde{L_{T}^{2}}\left(\mathcal{B}^{0,1 / 2}\right)}} \in \ell_{q}^{1} .
$$


Nous estimons le terme de reste comme suit. D'après l'inégalité (3.3) du lemme 3.2 , on a

$$
\begin{aligned}
\left\|\triangle_{q}^{\mathrm{v}} R\left(u, \nabla_{h} v\right)\right\|_{L_{\mathrm{v}}^{2}} & \leq 2^{q / 2}\left\|\triangle_{q}^{\mathrm{v}} R\left(u, \nabla_{h} v\right)\right\|_{L_{\mathrm{v}}^{1}} \\
& \leq 2^{q / 2} \sum_{\substack{q^{\prime}>q-4 \\
i \in\{0, \pm 1\}}}\left\|\triangle_{q^{\prime}}^{\mathrm{v}} u\right\|_{L_{\mathrm{v}}^{2}}\left\|\triangle_{q^{\prime}-i}^{\mathrm{v}} \nabla_{h} v\right\|_{L_{\mathrm{v}}^{2}}
\end{aligned}
$$

En prenant la norme $L_{h}^{4 / 3}$, nous obtenons

$$
\begin{aligned}
& \| \triangle_{q}^{\mathrm{v}} R\left(u, \nabla_{h} v\right)\left\|_{L_{h}^{4 / 3}\left(L_{\mathrm{v}}^{2}\right)} \leq 2^{q / 2} \sum_{\substack{q^{\prime}>q-4 \\
i \in\{0, \pm 1\}}}\right\| \triangle_{q^{\prime}}^{\mathrm{v}} u\left\|_{L_{h}^{4}\left(L_{\mathrm{v}}^{2}\right)}\right\| \triangle_{q^{\prime}-i}^{\mathrm{v}} \nabla_{h} v \|_{L^{2}\left(\mathbb{R}^{3}\right)} \\
& \leq 2^{q / 2} \sum_{\substack{q^{\prime}>q-4 \\
i \in\{0, \pm 1\}}}\left\|\triangle_{q^{\prime}}^{\mathrm{v}} u\right\|_{L^{2}\left(\mathbb{R}^{3}\right)}^{1 / 2}\left\|\triangle_{q^{\prime}}^{\mathrm{v}} \nabla_{h} u\right\|_{L^{2}\left(\mathbb{R}^{3}\right)}^{1 / 2}\left\|\triangle_{q^{\prime}-i}^{\mathrm{v}} \nabla_{h} v\right\|_{L^{2}\left(\mathbb{R}^{3}\right)} .
\end{aligned}
$$

En prenant maintenant la norme $L_{T}^{4 / 3}$, on a

$$
\begin{aligned}
\| \triangle_{q}^{\mathrm{v}} R & \left(u, \nabla_{h} v\right) \|_{L_{T}^{4 / 3}\left(L_{h}^{4 / 3} L_{\mathrm{v}}^{2}\right)} \leq \\
& \leq 2^{q / 2} \sum_{\substack{q^{\prime}>q-4 \\
i \in\{0, \pm 1\}}}\left\|\triangle_{q^{\prime}}^{\mathrm{v}} u\right\|_{L_{T}^{\infty}\left(L^{2}\right)}^{1 / 2}\left\|\triangle_{q^{\prime}}^{\mathrm{v}} \nabla_{h} u\right\|_{L_{T}^{2}\left(L^{2}\right)}^{1 / 2}\left\|\triangle_{q^{\prime}-i}^{\mathrm{v}} \nabla_{h} v\right\|_{L_{T}^{2}\left(L^{2}\right)} \\
& \leq 2^{q / 2} \sum_{\substack{q^{\prime}>q-4 \\
i \in\{0, \pm 1\}}} 2^{-q^{\prime}} \tilde{a}_{q^{\prime}}\|u\|_{\widetilde{L_{T}^{\infty}\left(\mathcal{B}^{0,1 / 2}\right)}}^{1 / 2}\left\|\nabla_{h} u\right\|_{\widetilde{L_{T}^{2}\left(\mathcal{B}^{0,1 / 2}\right)}}^{1 / 2}\left\|\nabla_{h} v\right\|_{\widetilde{L}_{T}^{2}\left(\mathcal{B}^{0,1 / 2}\right)}
\end{aligned}
$$

où $\tilde{a}_{q}=b_{q}^{(2)} \sqrt{\tilde{b}_{q}^{(1)} \tilde{b}_{q}^{(2)}}$, avec

$$
\tilde{b}_{q}^{(1)}=\frac{2^{q / 2}\left\|\triangle_{q}^{\mathrm{v}} u\right\|_{L_{T}^{\infty}\left(L^{2}\right)}}{\|u\|_{\widetilde{L_{T}^{\infty}}\left(\mathcal{B}^{0,1 / 2}\right)}} \in \ell_{q}^{1}, \quad \tilde{b}_{q}^{(2)}=\frac{2^{q / 2}\left\|\triangle_{q}^{\mathrm{v}} \nabla_{h} u\right\|_{L_{T}^{2}\left(L^{2}\right)}}{\left\|\nabla_{h} u\right\|_{\widetilde{L_{T}^{2}}\left(\mathcal{B}^{0,1 / 2}\right)}} \in \ell_{q}^{1},
$$

et

$$
b_{q}^{(2)}=\frac{2^{q / 2}\left\|\triangle_{q}^{v} \nabla_{h} v\right\|_{L_{T}^{2}\left(L^{2}\right)}}{\left\|\nabla_{h} v\right\|_{\widetilde{L}_{T}^{2}\left(\mathcal{B}^{0,1 / 2}\right)}} \in \ell_{q}^{1} .
$$

On a $\sum_{q} \tilde{a}_{q}^{1 / 2} \leq 1$ donc, en particulier $\tilde{a}_{q} \in \ell_{q}^{1}$.

Si on note $\bar{b}_{q}=2^{q} \sum_{q^{\prime} \geq q-N_{0}} 2^{-q^{\prime}} \tilde{a}_{q}$, alors $\bar{b}_{q} \in \ell_{q}^{1}$ et on a

$$
\begin{aligned}
& \left\|\triangle_{q}^{\mathrm{v}} R\left(u, \nabla_{h} v\right)\right\|_{L_{T}^{4 / 3}\left(L_{h}^{4 / 3} L_{\mathrm{v}}^{2}\right)} \leq \\
& \quad \leq C 2^{-q / 2} \bar{b}_{q}\|u\|_{\widetilde{L_{T}^{\infty}\left(\mathcal{B}^{0,1 / 2}\right)}}^{1 / 2}\left\|\nabla_{h} u\right\|_{\widetilde{L_{T}^{2}\left(\mathcal{B}^{0,1 / 2}\right)}}^{1 / 2}\left\|\nabla_{h} v\right\|_{\widetilde{L_{T}^{2}\left(\mathcal{B}^{0,1 / 2}\right)}} .
\end{aligned}
$$


En sommant les estimations (5.2), (5.3) et (5.4) et en utilisant la relation (5.1), nous obtenons

$$
\left\|F_{q}^{h}\right\|_{L_{T}^{4 / 3}\left(L_{h}^{4 / 3} L_{\mathrm{v}}^{2}\right)} \leq C 2^{-q / 2} b_{q}\|u\|_{\widetilde{L_{T}^{\infty}\left(\mathcal{B}^{0,1 / 2}\right)}}^{1 / 2}\left\|\nabla_{h} u\right\|_{\widetilde{L_{T}^{2}\left(\mathcal{B}^{0,1 / 2}\right)}}^{1 / 2}\left\|\nabla_{h} v\right\|_{\widetilde{L_{T}^{2}\left(\mathcal{B}^{0,1 / 2}\right)}}
$$

où $b_{q} \in \ell_{q}^{1}$. Donc, nous avons

$$
\begin{aligned}
& \int_{0}^{T}\left|\left(F_{q}^{h}(\tau) \mid \triangle_{q}^{\mathrm{v}} v(\tau)\right)_{L^{2}}\right| d \tau \leq \\
& (5.5) \quad \leq 2^{-q} C a_{q}\|u\|_{\widetilde{L}_{T}^{\infty}\left(\mathcal{B}^{0,1 / 2}\right)}^{1 / 2}\left\|\nabla_{h} u\right\|_{\widetilde{L}_{T}^{2}\left(\mathcal{B}^{0,1 / 2}\right)}^{1 / 2}\|v\|_{\widetilde{L}_{T}^{\infty}\left(\mathcal{B}^{0,1 / 2}\right)}^{1 / 2}\left\|\nabla_{h} v\right\|_{\widetilde{L}_{T}^{2}\left(\mathcal{B}^{0,1 / 2}\right)}^{3 / 2}
\end{aligned}
$$

avec $a_{q} \in \ell_{q}^{1 / 2}$.

Pour estimer $\int_{0}^{T}\left|\left(F_{q}^{\mathrm{v}}(\tau) \mid \triangle_{q}^{\mathrm{v}} v(\tau)\right)_{L^{2}}\right| d \tau$ nous allons décomposer comme dans [6]. On a

$$
\begin{aligned}
& \triangle_{q}^{\mathrm{v}}\left(u_{3} \cdot \partial_{3} v\right)=S_{q-1}^{\mathrm{v}} u_{3} \cdot \partial_{3} \triangle_{q}^{\mathrm{v}} v+\sum_{\left|q^{\prime}-q\right| \leq 4}\left[\triangle_{q}^{\mathrm{v}} ; S_{q^{\prime}-1}^{\mathrm{v}} u_{3}\right] \partial_{3} \triangle_{q^{\prime}}^{\mathrm{v}} v \\
& +\sum_{\left|q^{\prime}-q\right| \leq 4}\left(S_{q-1}^{\mathrm{v}} u_{3}-S_{q^{\prime}-1}^{\mathrm{v}} u_{3}\right) \cdot \partial_{3} \triangle_{q}^{\mathrm{v}} \triangle_{q^{\prime}}^{\mathrm{v}} v+\sum_{q^{\prime}>q-4} \triangle_{q}^{\mathrm{v}}\left(S_{q^{\prime}+1}^{\mathrm{v}}\left(\partial_{3} v\right) \cdot \triangle_{q^{\prime}}^{\mathrm{v}} u_{3}\right)
\end{aligned}
$$

En tenant compte du fait que $u(t)$ est de divergence nulle, ce qui nous permet de transformer la dérivée verticale en dérivée horizontale, on obtient après intégration par parties

$$
\int_{\mathbb{R}^{3}} S_{q-1}^{\mathrm{v}} u_{3} \partial_{3} \triangle_{q}^{\mathrm{v}} v \cdot \triangle_{q}^{\mathrm{v}} v d x=\frac{1}{2} \int_{\mathbb{R}^{3}} S_{q-1}^{\mathrm{v}}\left(\operatorname{div}_{h} u^{h}\right) \triangle_{q}^{\mathrm{v}} v \cdot \triangle_{q}^{\mathrm{v}} v d x .
$$

Par une intégration en temps, il vient

$$
\int_{0}^{T}\left|\left(S_{q-1}^{\mathrm{v}} u_{3} \partial_{3} \triangle_{q}^{\mathrm{v}} v \mid \triangle_{q}^{\mathrm{v}} v\right)_{L^{2}}\right| d \tau=\frac{1}{2} \int_{0}^{T}\left|\left(S_{q-1}^{\mathrm{v}}\left(\operatorname{div}_{h} u^{h}\right) \triangle_{q}^{\mathrm{v}} v \mid \triangle_{q}^{\mathrm{v}} v\right)_{L^{2}}\right| d \tau
$$

En utilisant l'inégalité de Hölder, on obtient :

$$
\begin{aligned}
\int_{0}^{T} \mid\left(S_{q-1}^{\mathrm{v}} u_{3} \cdot \partial_{3}\right. & \left.\triangle_{q}^{\mathrm{v}} v \mid \triangle_{q}^{\mathrm{v}} v\right)_{L^{2}} \mid d \tau \leq \\
& \leq C \int_{0}^{T}\left\|S_{q-1}^{\mathrm{v}}\left(\nabla_{h} u\right)\right\|_{L_{\mathrm{v}}^{\infty} L_{h}^{2}}\left\|\triangle_{q}^{\mathrm{v}} v\right\|_{L_{\mathrm{v}}^{2} L_{h}^{4}}\left\|\triangle_{q}^{\mathrm{v}} v\right\|_{L_{\mathrm{v}}^{2} L_{h}^{4}} d \tau \\
& \leq C \int_{0}^{T}\left\|\nabla_{h} u(\tau)\right\|_{\mathcal{B}^{0,1 / 2}}\left\|\triangle_{q}^{\mathrm{v}} v(\tau)\right\|_{L_{\mathrm{v}}^{2} L_{h}^{4}}^{2} d \tau
\end{aligned}
$$


En utilisant le lemme 3.3, nous obtenons

$$
\begin{aligned}
& \int_{0}^{T} \mid\left(S_{q-1}^{\mathrm{v}} u_{3} \cdot\right.\left.\partial_{3} \triangle_{q}^{\mathrm{v}} v \mid \triangle_{q}^{\mathrm{v}} v\right)_{L^{2}} \mid d \tau \leq \\
& \leq C \int_{0}^{T}\left\|\nabla_{h} u(\tau)\right\|_{\mathcal{B}^{0,1 / 2}}\left\|\triangle_{q}^{\mathrm{v}} v(\tau)\right\|_{L^{2}}\left\|\triangle_{q}^{\mathrm{v}} \nabla_{h} v(\tau)\right\|_{L^{2}} d \tau \\
& \leq 2^{-q} C a_{q}\left\|\nabla_{h} u\right\|_{L_{T}^{2}\left(\mathcal{B}^{0,1 / 2}\right)}\|v\|_{\widetilde{L_{T}^{\infty}\left(\mathcal{B}^{0,1 / 2}\right)}}\left\|\nabla_{h} v\right\|_{\widetilde{L_{T}^{2}\left(\mathcal{B}^{0,1 / 2}\right)}}
\end{aligned}
$$

avec $a_{q}=b_{q}^{(1)} b_{q}^{(2)} \quad$ où on a noté

$b_{q}^{(1)}=\frac{2^{q / 2}\left\|\triangle_{q}^{\mathrm{v}} v\right\|_{L_{T}^{\infty}\left(L^{2}\right)}}{\|v\|_{\widetilde{L_{T}^{\infty}}\left(\mathcal{B}^{0,1 / 2}\right)}} \in \ell_{q}^{1}$ respectivement $b_{q}^{(2)}=\frac{2^{q / 2}\left\|\triangle_{q}^{\mathrm{v}} \nabla_{h} v\right\|_{L_{T}^{2}\left(L^{2}\right)}}{\left\|\nabla_{h} v\right\|_{\widetilde{L_{T}^{2}}\left(\mathcal{B}^{0,1 / 2}\right)}} \in \ell_{q}^{1}$ et donc $a_{q} \in \ell_{q}^{1 / 2}$.

Pour estimer le terme qui contient le commutateur, nous utilisons l'inégalité (3.8) du lemme 3.3 sous la forme suivante:

$$
\left\|\left[\triangle_{q}^{\mathrm{v}} ; u\right] v\right\|_{L_{\mathrm{v}}^{2}\left(L_{h}^{p}\right)} \leq C 2^{-q}\left\|\partial_{3} u\right\|_{L_{\mathrm{v}}^{\infty}\left(L_{h}^{r}\right)}\|v\|_{L_{\mathrm{v}}^{2}\left(L_{h}^{s}\right)}
$$

pour $p \geq 1 ; r \geq 1 ; s \geq 1$ avec $1 / p=1 / r+1 / s$.

Dans notre cas, l'estimation donne

$$
\begin{aligned}
& \sum_{\left|q^{\prime}-q\right| \leq 4} \int_{0}^{t}\left|\left(\left[\triangle_{q}^{\mathrm{v}} ; S_{q^{\prime}-1}^{\mathrm{v}} u_{3}\right] \partial_{3} \triangle_{q^{\prime}}^{\mathrm{v}} v \mid \triangle_{q}^{\mathrm{v}} v\right)_{L^{2}}\right| d \tau \leq \\
& \quad \leq \sum_{\left|q^{\prime}-q\right| \leq 4} \int_{0}^{T}\left\|\left[\triangle_{q}^{\mathrm{v}} ; S_{q^{\prime}-1}^{\mathrm{v}} u_{3}\right] \partial_{3} \triangle_{q^{\prime}}^{\mathrm{v}} v\right\|_{L_{\mathrm{v}}^{2}\left(L_{h}^{4 / 3}\right)}\left\|\triangle_{q}^{\mathrm{v}} v\right\|_{L_{\mathrm{v}}^{2} L_{h}^{4}} d \tau \\
& \quad \leq C \sum_{\left|q^{\prime}-q\right| \leq 4} 2^{-q} \int_{0}^{T}\left\|S_{q^{\prime}-1}^{\mathrm{v}} \partial_{3} u_{3}\right\|_{L_{\mathrm{v}}^{\infty} L_{h}^{2}}\left\|\partial_{3} \triangle_{q^{\prime}}^{\mathrm{v}} v\right\|_{L_{\mathrm{v}}^{2} L_{h}^{4}}\left\|\triangle_{q}^{\mathrm{v}} v\right\|_{L_{\mathrm{v}}^{2} L_{h}^{4}} d \tau \\
& \quad \leq C \sum_{\left|q^{\prime}-q\right| \leq 4} 2^{-q}\left\|S_{q^{\prime}-1}^{\mathrm{v}} \partial_{3} u_{3}\right\|_{L_{T}^{2}\left(L_{\mathrm{v}}^{\infty} L_{h}^{2}\right)}\left\|\partial_{3} \triangle_{q^{\prime}}^{\mathrm{v}} v\right\|_{L_{T}^{4}\left(L_{\mathrm{v}}^{2} L_{h}^{4}\right)}\left\|\triangle_{q}^{\mathrm{v}} v\right\|_{L_{T}^{4}\left(L_{\mathrm{v}}^{2} L_{h}^{4}\right)}
\end{aligned}
$$

Compte tenu qu'on travaille avec des fonctions localisées en fréquences verticales, on a

$$
\left\|\partial_{3} \triangle_{q^{\prime}}^{\mathrm{v}} v\right\|_{L_{T}^{4}\left(L_{\mathrm{v}}^{2} L_{h}^{4}\right)} \leq C 2^{q^{\prime}}\left\|\triangle_{q^{\prime}}^{\mathrm{v}} v\right\|_{L_{T}^{4}\left(L_{\mathrm{v}}^{2} L_{h}^{4}\right)} \leq C 2^{q^{\prime}}\left\|\triangle_{q^{\prime}}^{\mathrm{v}} v\right\|_{L_{T}^{\infty}\left(L^{2}\right)}^{1 / 2}\left\|\triangle_{q^{\prime}}^{\mathrm{v}} \nabla_{h} v\right\|_{L_{T}^{2}\left(L^{2}\right)}^{1 / 2} .
$$

Comme $\partial_{3} u_{3}=-\operatorname{div}_{h} u^{h}$, nous obtenons que

$$
\begin{aligned}
& \sum_{\left|q^{\prime}-q\right| \leq 4} \int_{0}^{T}\left|\left(\left[\triangle_{q}^{\mathrm{v}} ; S_{q^{\prime}-1}^{\mathrm{v}} u_{3}\right] \partial_{3} \triangle_{q^{\prime}}^{\mathrm{v}} v \mid \triangle_{q}^{\mathrm{v}} v\right)_{L^{2}}\right| d \tau \leq \\
& \quad \leq C 2^{-q} a_{q}\left\|\nabla_{h} u\right\|_{L_{T}^{2}\left(\mathcal{B}^{0,1 / 2}\right)}\|v\|_{\widetilde{L_{T}^{\infty}\left(\mathcal{B}^{0,1 / 2}\right)}}\left\|\nabla_{h} v\right\|_{\widetilde{L_{T}^{2}\left(\mathcal{B}^{0,1 / 2}\right)}}
\end{aligned}
$$

avec $a_{q} \in l_{q}^{1 / 2}$. 
Les estimations pour $\int_{0}^{T}\left|\left(\left(S_{q^{\prime}-1}^{\mathrm{v}} u_{3}-S_{q-1}^{\mathrm{v}} u_{3}\right) \partial_{3} \triangle_{q}^{\mathrm{v}} \triangle_{q^{\prime}}^{\mathrm{v}} v \mid \triangle_{q}^{\mathrm{v}} v\right)_{L^{2}}\right| d \tau$ sont analogues aux précédentes, si nous observons que pour $\left|q^{\prime}-q\right| \leq 1$ on a que $\left(S_{q^{\prime}-1}^{\mathrm{v}} u_{3}-S_{q-1}^{\mathrm{v}} u_{3}\right)$ est soit la fonction nulle soit $u_{3}$ localisé sur une couronne de taille $2^{q}$. À nouveau, la condition de divergence nulle nous permet de transformer une dérivée verticale en dérivée horizontale.

Le dernier terme à estimer est

$$
\sum_{q^{\prime}>q-4} \int_{0}^{T}\left|\left(\triangle_{q}^{\mathrm{v}}\left(S_{q^{\prime}+1}^{\mathrm{v}}\left(\partial_{3} v\right) \cdot \triangle_{q^{\prime}}^{\mathrm{v}} u_{3}\right) \mid \triangle_{q}^{\mathrm{v}} v\right)_{L^{2}}\right| d \tau .
$$

En utilisant de nouveau les lois de produit dans les espaces de Lebesgue sur la variable horizontale et l'inégalité de Hölder dans la variable verticale ainsi qu'en temps, on majore ce terme par

$$
\sum_{q^{\prime}>q-4}\left\|S_{q^{\prime}+1}^{\mathrm{v}}\left(\partial_{3} v\right)\right\|_{L_{T}^{4}\left(L_{\mathrm{v}}^{\infty} L_{h}^{4}\right)}\left\|\triangle_{q^{\prime}}^{\mathrm{v}} u_{3}\right\|_{L_{T}^{2}\left(L_{\mathrm{v}}^{2} L_{h}^{2}\right)}\left\|\triangle_{q}^{\mathrm{v}} v\right\|_{L_{T}^{4}\left(L_{\mathrm{v}}^{2} L_{h}^{4}\right)} .
$$

L'inégalité (3.2) du lemme 3.2 et le corollaire 2, nous donne

$$
\left\|S_{q^{\prime}+1}^{\mathrm{v}}\left(\partial_{3} v\right)\right\|_{L_{T}^{4}\left(L_{\mathrm{v}}^{\infty} L_{h}^{4}\right)} \leq C 2^{q^{\prime}}\|v\|_{L_{T}^{4}\left(L_{\mathrm{v}}^{\infty} L_{h}^{4}\right)} \leq C 2^{q^{\prime}}\|v\|_{L_{T}^{\infty}\left(\mathcal{B}^{0,1 / 2}\right)}^{1 / 2}\left\|\nabla_{h} v\right\|_{L_{T}^{2}\left(\mathcal{B}^{0,1 / 2}\right)}^{1 / 2} .
$$

D'après le lemme 3.2 , on a

$$
\left\|\triangle_{q^{\prime}}^{\mathrm{v}} u_{3}\right\|_{L^{2}\left([0, T] \times \mathbb{R}^{3}\right)} \leq C 2^{-q^{\prime}}\left\|\triangle_{q^{\prime}}^{\mathrm{v}} \partial_{3} u_{3}\right\|_{L^{2}\left([0, T] \times \mathbb{R}^{3}\right)} .
$$

On utilise à nouveau la condition de divergence nulle pour dire que $\partial_{3} u_{3}=$ $-\operatorname{div}{ }_{h} u_{h}$ et on obtient

$$
\left\|\triangle_{q^{\prime}}^{\mathrm{v}} u_{3}\right\|_{L^{2}\left([0, T] \times \mathbb{R}^{3}\right)} \leq C 2^{-q^{\prime}}\left\|\triangle_{q^{\prime}}^{\mathrm{v}} \nabla_{h} u\right\|_{L_{T}^{2}\left(L^{2}\left(\mathbb{R}^{3}\right)\right)} .
$$

Il en résulte que

$$
\begin{aligned}
\sum_{q^{\prime}>q-4} & \int_{0}^{T}\left|\left(\triangle_{q}^{\mathrm{v}}\left(S_{q^{\prime}+1}^{\mathrm{v}}\left(\partial_{3} v\right) \cdot \triangle_{q^{\prime}}^{\mathrm{v}} u_{3}\right) \mid \triangle_{q}^{\mathrm{v}} v\right)\right| d \tau \\
\leq & C\|v\|_{L_{T}^{\infty}\left(\mathcal{B}^{0,1 / 2}\right)}^{1 / 2}\left\|\nabla_{h} v\right\|_{L_{T}^{2}\left(\mathcal{B}^{0,1 / 2}\right)}^{1 / 2} \times \\
& \times \sum_{q^{\prime}>q-4}\left\|\triangle_{q^{\prime}}^{\mathrm{v}} \nabla_{h} u\right\|_{L_{T}^{2}\left(L^{2}\left(\mathbb{R}^{3}\right)\right)}\left\|\triangle_{q}^{\mathrm{v}} v\right\|_{L_{T}^{\infty}\left(L^{2}\right)}^{1 / 2}\left\|\triangle_{q}^{\mathrm{v}} \nabla_{h} v\right\|_{L_{T}^{2}\left(L^{2}\right)}^{1 / 2} \\
& \leq C 2^{-q / 2} b_{q}\|v\|_{L_{T}^{\infty}\left(\mathcal{B}^{0,1 / 2}\right)}\left\|\nabla_{h} v\right\|_{L_{T}^{2}\left(\mathcal{B}^{0,1 / 2}\right)} \sum_{q^{\prime} \geq q-4} 2^{-q^{\prime} / 2 \tilde{b}_{q^{\prime}}^{(2)}}\left\|\nabla_{h} u\right\|_{L_{T}^{2}\left(\mathcal{B}^{0,1 / 2}\right)} \\
\text { où } \bar{b}_{q}= & 2^{q / 2} \sum_{q^{\prime}>q-4} 2^{-q^{\prime} / 2 \tilde{b}_{q^{\prime}}^{(2)}} ; \bar{b}_{q} \in \ell_{q}^{1}, \text { et } b_{q} \in \ell_{q}^{1} \text {. Donc, } a_{q}=b_{q} \bar{b}_{q} \in \|_{\ell_{q}^{1 / 2}} .
\end{aligned}
$$

En sommant les estimations (5.7)-(5.9) et en utilisant les relations (5.6) et (5.5), on peut conclure la démonstration du lemme 4.1 . 


\subsection{L'estimation pour l'unicité}

Soient $u$ et $v$ deux champs de divergence nulle appartenant à l'espace $L_{T}^{\infty}\left(H^{0,1 / 2}\right)$ avec $\nabla_{h} u$ et $\nabla_{h} v$ dans $L_{T}^{2}\left(H^{0,1 / 2}\right)$.

Soit $w$ dans $L_{T}^{\infty}\left(H^{0,1 / 2}\right)$ avec $\nabla_{h} w$ appartenant à l'espace $H^{0,1 / 2}$ champ de divergence nulle vérifiant l'équation

$$
\partial_{t} w+u \cdot \nabla w+w \cdot \nabla v-\nu_{h} \Delta_{h} w=-\nabla p .
$$

Nous allons démontrer dans la suite l'estimation (2.2). Plus précisément on va démontrer le lemme 4.2.

Démonstration. Pour démontrer ce lemme, on procède par estimation d'énergie sur l'équation vérifiée par $w$. On a

$$
\begin{aligned}
\frac{1}{2} \frac{d}{d t}\|w(t)\|_{H^{0,-1 / 2}}^{2}+\nu_{h}\left\|\nabla_{h} w(t)\right\|_{H^{0,-1 / 2}}^{2} & \leq \sum_{q \geq-1} 2^{-q} \int \triangle_{q}^{\mathrm{v}}(u \cdot \nabla w) \triangle_{q}^{\mathrm{v}} w d x \\
& +\sum_{q \geq-1} 2^{-q} \int \triangle_{q}^{\mathrm{v}}(w \cdot \nabla v) \triangle_{q}^{\mathrm{v}} w d x
\end{aligned}
$$

Nous allons estimer d'abord les termes horizontaux, c'est-à-dire les termes ne contenant que des dérivées dans les variables horizontales. La décomposition de Bony dans la variable verticale nous donne

$$
\sum_{q \geq-1} 2^{-q} \int \triangle_{q}^{\mathrm{v}}\left(u_{h} \cdot \nabla_{h} w\right) \triangle_{q}^{\mathrm{v}} w d x=T_{1}^{h}+T_{2}^{h}+R^{h},
$$

où on a noté

$$
\begin{aligned}
& T_{1}^{h}=\sum_{q \geq-1} 2^{-q} \sum_{\left|q-q^{\prime}\right| \leq 4} \int \triangle_{q}^{\mathrm{v}}\left(S_{q^{\prime}-1}^{\mathrm{v}} \nabla_{h} w \cdot \triangle_{q^{\prime}}^{\mathrm{v}} u_{h}\right) \triangle_{q}^{\mathrm{v}} w d x, \\
& T_{2}^{h}=\sum_{q \geq-1} 2^{-q} \sum_{\left|q-q^{\prime}\right| \leq 4} \int \triangle_{q}^{\mathrm{v}}\left(S_{q^{\prime}-1}^{\mathrm{v}} u_{h} \cdot \nabla_{h} \triangle_{q^{\prime}}^{\mathrm{v}} w\right) \triangle_{q}^{\mathrm{v}} w d x \quad \text { et } \\
& R^{h}=\sum_{q \geq-1} 2^{-q} \sum_{\substack{i \in\{0, \pm 1\} \\
q^{\prime}>q-4}} \int \triangle_{q}^{\mathrm{v}}\left(\triangle_{q^{\prime}}^{\mathrm{v}} \nabla_{h} w \cdot \triangle_{q^{\prime}+i}^{\mathrm{v}} u_{h}\right) \triangle_{q}^{\mathrm{v}} w d x .
\end{aligned}
$$

Pour le premier terme $T_{1}^{h}$, on a l'estimation

$$
T_{1}^{h} \leq \sum_{q \geq-1} \sum_{\left|q-q^{\prime}\right| \leq 4} 2^{-q}\left\|S_{q^{\prime}-1}^{\mathrm{v}} \nabla_{h} w\right\|_{L_{\mathrm{v}}^{\infty} L_{h}^{2}}\left\|\triangle_{q^{\prime}}^{\mathrm{v}} u\right\|_{L_{\mathrm{v}}^{2} L_{h}^{4}}\left\|\triangle_{q}^{\mathrm{v}} w\right\|_{L_{\mathrm{v}}^{2} L_{h}^{4}}
$$


En utilisant le lemme 3.3, l'inégalité (3.7), on obtient

$$
\begin{aligned}
T_{1}^{h} \leq \sum_{q \geq-1} 2^{-q} \sum_{\left|q-q^{\prime}\right| \leq 4} & \left\|S_{q^{\prime}-1}^{\mathrm{v}} \nabla_{h} w\right\|_{L_{\mathrm{v}}^{\infty} L_{h}^{2}}\left\|\triangle_{q^{\prime}}^{\mathrm{v}} u\right\|_{L^{2}}^{1 / 2} \times \\
& \times\left\|\triangle_{q^{\prime}}^{\mathrm{v}} \nabla_{h} u\right\|_{L^{2}}^{1 / 2}\left\|\triangle_{q}^{\mathrm{v}} w\right\|_{L^{2}}^{1 / 2}\left\|\triangle_{q}^{\mathrm{v}} \nabla_{h} w\right\|_{L^{2}}^{1 / 2} .
\end{aligned}
$$

Parce que $\nabla_{h} w \in H^{0,-1 / 2}$, on a

$$
\left\|S_{q-1}^{\mathrm{v}} \nabla_{h} w\right\|_{L_{\mathrm{v}}^{\infty} L_{h}^{2}} \leq C 2^{q}\left\|\nabla_{h} w\right\|_{H^{0,-1 / 2}}
$$

Parce que $u \in L_{T}^{\infty}\left(H^{0,1 / 2}\right)$ et $\nabla_{h} u \in L_{T}^{2}\left(H^{0,1 / 2}\right)$, il existe une suite de carré sommable $c_{q}(t)$ telle que

$$
\left\|\triangle_{q}^{\mathrm{v}} u(t)\right\|_{L^{2}}^{1 / 2}\left\|\triangle_{q}^{\mathrm{v}} \nabla_{h} u(t)\right\|_{L^{2}}^{1 / 2} \leq C 2^{-q / 2} c_{q}(t)\|u(t)\|_{H^{0,1 / 2}}^{1 / 2}\left\|\nabla_{h} u(t)\right\|_{H^{0,1 / 2}}^{1 / 2} .
$$

Comme on estime $w$ dans l'espace $H^{0,-1 / 2}$, il existe une suite de carré sommable $c_{q}(t)$ telle que

$$
\left\|\triangle_{q}^{\mathrm{v}} w(t)\right\|_{L^{2}}^{1 / 2}\left\|\triangle_{q}^{\mathrm{v}} \nabla_{h} w(t)\right\|_{L^{2}}^{1 / 2} \leq 2^{q / 2} c_{q}(t)\|w(t)\|_{H^{0,-1 / 2}}^{1 / 2}\left\|\nabla_{h} w(t)\right\|_{H^{0,-1 / 2}}^{1 / 2}
$$

Donc on obtient

$$
T_{1}^{h} \leq \sum_{q \geq-1} C b_{q}\left\|\nabla_{h} w\right\|_{H^{0,-1 / 2}}\|u\|_{H^{0,1 / 2}}^{1 / 2}\left\|\nabla_{h} u\right\|_{H^{0,1 / 2}}^{1 / 2}\|w\|_{H^{0,-1 / 2}}^{1 / 2}\left\|\nabla_{h} w\right\|_{H^{0,-1 / 2}}^{1 / 2},
$$

avec $b_{q} \in \ell^{1}(\mathbb{N})$. Nous avons alors

$$
\begin{aligned}
T_{1}^{h} & \leq C\|u\|_{H^{0,1 / 2}}^{1 / 2}\left\|\nabla_{h} u\right\|_{H^{0,1 / 2}}^{1 / 2}\|w\|_{H^{0,-1 / 2}}^{1 / 2}\left\|\nabla_{h} w\right\|_{H^{0,-1 / 2}}^{3 / 2} \\
& \leq\left(100 / \nu_{h}^{3}\right) C\|u\|_{H^{0,1 / 2}}^{2}\left\|\nabla_{h} u\right\|_{H^{0,1 / 2}}^{2}\|w\|_{H^{0,-1 / 2}}^{2}+\frac{\nu_{h}}{100}\left\|\nabla_{h} w\right\|_{H^{0,-1 / 2}}^{2} \\
(5.10) & \leq C f(t)\|w\|_{H^{0,-1 / 2}}^{2}+\frac{\nu_{h}}{100}\left\|\nabla_{h} w\right\|_{H^{0,1 / 2}}^{2}
\end{aligned}
$$

où par $f$ on va désigner dans toute la suite, la fonction localement intégrable donnée par la rélation (4.1) du lemme 4.2.

Le deuxième terme est plus délicat. Soit $N$ un paramètre qui sera fixé à la fin. On écrit la décomposition $T_{2}^{h}=T_{2, N}^{h}+T_{2}^{h, N}$, avec

$$
\begin{aligned}
& T_{2, N}^{h}=\sum_{q \leq N} 2^{-q} \sum_{\left|q-q^{\prime}\right| \leq 4} \int \triangle_{q}^{\mathrm{v}}\left(S_{q^{\prime}-1}^{\mathrm{v}} u_{h} \cdot \nabla_{h} \triangle_{q^{\prime}}^{\mathrm{v}} w\right) \triangle_{q}^{\mathrm{v}} w d x \quad \text { et } \\
& T_{2}^{h, N}=\sum_{q \geq N} 2^{-q} \sum_{\left|q-q^{\prime}\right| \leq 4} \int \triangle_{q}^{\mathrm{v}}\left(S_{q^{\prime}-1}^{\mathrm{v}} u_{h} \cdot \nabla_{h} \triangle_{q^{\prime}}^{\mathrm{v}} w\right) \triangle_{q}^{\mathrm{v}} w d x .
\end{aligned}
$$


Les deux sommes sont traitées de manière différente. Estimons d'abord $T_{2}^{h, N}$. Parce que $u \in H^{0,1 / 2}$, on a

$$
\left\|S_{q-1}^{\mathrm{v}} u\right\|_{L_{\mathrm{v}}^{\infty} L_{h}^{4}} \leq\left\|S_{q-1}^{\mathrm{v}} u\right\|_{L_{\mathrm{v}}^{\infty} L_{h}^{2}}^{1 / 2}\left\|S_{q-1}^{\mathrm{v}} \nabla_{h} u\right\|_{L_{\mathrm{v}}^{\infty} L_{h}^{2}}^{1 / 2} \leq C \sqrt{q}\|u\|_{H^{0,1 / 2}}^{1 / 2}\left\|\nabla_{h} u\right\|_{H^{0,1 / 2}}^{1 / 2}
$$

On obtient alors

$$
\begin{aligned}
& T_{2}^{h, N} \leq \sum_{q \geq N} 2^{-q} \sum_{\left|q-q^{\prime}\right| \leq 4} \| S_{q^{\prime}-1}^{\mathrm{v}} u_{h}\left\|_{L_{\mathrm{v}}^{\infty} L_{h}^{4}}\right\| \nabla_{h} \triangle_{q^{\prime}}^{\mathrm{v}} w\left\|_{L^{2}}\right\| \triangle_{q}^{\mathrm{v}} w \|_{L_{\mathrm{v}}^{2} L_{h}^{4}} \\
& \leq C \sum_{q \geq N} 2^{-q} \sum_{\left|q-q^{\prime}\right| \leq 4} \sqrt{q^{\prime}}\|u\|_{H^{0,1 / 2}}^{1 / 2}\left\|\nabla_{h} u\right\|_{H^{0,1 / 2}}^{1 / 2} \times \\
& \times\left\|\nabla_{h} \triangle_{q^{\prime}}^{\mathrm{v}} w\right\|_{L^{2}}\left\|\triangle_{q}^{\mathrm{v}} w\right\|_{L^{2}}^{1 / 2}\left\|\nabla_{h} \triangle_{q}^{\mathrm{v}} w\right\|_{L^{2}}^{1 / 2} .
\end{aligned}
$$

En tenant compte du fait que $\nabla_{h} w$ appartient à l'espace $L_{T}^{2}\left(H^{0,1 / 2}\right)$, on a

$$
\left\|\nabla_{h} \triangle_{q^{\prime}}^{\mathrm{v}} w(t)\right\|_{L^{2}} \leq c_{q^{\prime}}(t) 2^{-q^{\prime} / 2}\left\|\nabla_{h} w(t)\right\|_{H^{0,1 / 2}}
$$

avec $c_{q^{\prime}}(t) \in L_{T}^{\infty}\left(\ell^{2}(\mathbb{N})\right)$. D'autre part, vu que la fonction $w$ est estimée dans l'espace $H^{0,-1 / 2}$, on a

$$
\left\|\triangle_{q}^{\mathrm{v}} w(t)\right\|_{L^{2}}^{1 / 2}\left\|\triangle_{q}^{\mathrm{v}} \nabla_{h} w(t)\right\|_{L^{2}}^{1 / 2} \leq 2^{q / 2} \tilde{c}_{q}(t)\|w(t)\|_{H^{0,-1 / 2}}^{1 / 2}\left\|\nabla_{h} w(t)\right\|_{H^{0,-1 / 2}}^{1 / 2},
$$

où $\tilde{c}_{q}(t) \in \ell^{2}(\mathbb{N})$. Par conséquent, on obtient

$$
\begin{gathered}
T_{2}^{h, N} \leq C \sum_{q \geq N} 2^{-q} \sqrt{q} b_{q}\|u\|_{H^{0,1 / 2}}^{1 / 2}\left\|\nabla_{h} u\right\|_{H^{0,1 / 2}}^{1 / 2}\left\|\nabla_{h} w\right\|_{H^{0,1 / 2}} \times \\
\times\|w\|_{H^{0,-1 / 2}}^{1 / 2}\left\|\nabla_{h} w\right\|_{H^{0,-1 / 2}}^{1 / 2}
\end{gathered}
$$

où

$$
b_{q}=\left(\sum_{\left|q-q^{\prime}\right| \leq 4} c_{q^{\prime}}\right) \tilde{c}_{q} \in \ell^{1}(\mathbb{N}) \quad \text { avec } \quad \sum_{q \geq-1} b_{q} \leq 1 .
$$

Comme $2^{-q / 2} \sqrt{q} \leq 1$, on a

$$
\begin{aligned}
T_{2}^{h, N} & \leq C 2^{-N / 2}\|u\|_{H^{0,1 / 2}}^{1 / 2}\left\|\nabla_{h} u\right\|_{H^{0,1 / 2}}^{1 / 2}\left\|\nabla_{h} w\right\|_{H^{0,1 / 2}}\|w\|_{H^{0,-1 / 2}}^{1 / 2}\left\|\nabla_{h} w\right\|_{H^{0,-1 / 2}}^{1 / 2} \\
& \leq C 2^{-2 N / 3} f(t)\|w\|_{H^{0,-1 / 2}}^{2 / 3}+\frac{\nu_{h}}{100}\left\|\nabla_{h} w\right\|_{H^{0,-1 / 2}}^{2} .
\end{aligned}
$$

Expliquons maintenant le choix de $N$. Afin de pouvoir utiliser sur ce terme le lemme de Gronwall, il faut prendre $N$ dans l'estimation ci-dessus tel que

$$
2^{-2 N / 3} C f(t)\|w\|_{H^{0,-1 / 2}}^{2 / 3}=C f(t)\|w(t)\|_{H^{0,-1 / 2}}^{2} .
$$

On obtient ainsi à une constante près $N=1-\ln \|w(t)\|_{H^{0,-1 / 2}}^{2}$. 
Estimons maintenant $T_{2, N}^{h}$. Afin d'éviter la difficulté montrée dans l'estimation (4.8), on va utiliser le moins possible de derivées dans la variable horizontale. Pour cela, on va faire appel au lemme 4.3, qui est un lemme de type "Gagliardo-Nirenberg" (voir [7]). Introduisons ainsi un nouveau paramètre $\varepsilon$ qui représente le nombre de dérivée horizontale utilisées. En revenant à l'estimation sur $T_{2, N}^{h}$, on va avoir à l'aide de l'inégalité de Hölder

$$
\begin{aligned}
T_{2, N}^{h} & \leq \sum_{q \leq N} 2^{-q} \sum_{\left|q-q^{\prime}\right| \leq 4}\left\|\triangle_{q}^{\mathrm{v}}\left(S_{q^{\prime}-1}^{\mathrm{v}} u^{h} \triangle_{q^{\prime}}^{\mathrm{v}} \nabla_{h} w\right)\right\|_{L_{\mathrm{v}}^{2}\left(L_{h}^{1+\varepsilon}\right)}\left\|\triangle_{q}^{\mathrm{v}} w\right\|_{L_{\mathrm{v}}^{2}\left(L_{h}^{1-\varepsilon}\right)} \\
& \leq \sum_{q \leq N} 2^{-q} \sum_{\left|q-q^{\prime}\right| \leq 4}\left\|S_{q^{\prime}-1}^{\mathrm{v}} u^{h}\right\|_{L_{\mathrm{v}}^{\infty}\left(L_{h}^{\frac{2}{\varepsilon}}\right)}\left\|\nabla_{h} \triangle_{q^{\prime}}^{\mathrm{v}} w\right\|_{L^{2}}\left\|\triangle_{q}^{\mathrm{v}} w\right\|_{L_{\mathrm{v}}^{2}\left(L_{h}^{\frac{2}{1-\varepsilon}}\right)}
\end{aligned}
$$

En utilisant maintenant l'inégalité donnée dans le lemme 4.3, on a pour tout $\varepsilon \in(0,1 / 2)$,

$$
\begin{aligned}
T_{2, N}^{h} \leq \sum_{q \leq N} 2^{-q} \frac{C}{\sqrt{\varepsilon}} \sum_{\left|q-q^{\prime}\right| \leq 4}\left\|S_{q^{\prime}-1}^{\mathrm{v}} u\right\|_{L_{\mathrm{v}}^{\infty} L_{h}^{2}}^{\varepsilon}\left\|S_{q^{\prime}-1}^{\mathrm{v}} \nabla_{h} u\right\|_{L_{\mathrm{v}}^{\infty} L_{h}^{2}}^{1-\varepsilon} \times \\
\times\left\|\nabla_{h} \triangle_{q^{\prime}}^{\mathrm{v}} w\right\|_{L^{2}}\left\|\triangle_{q}^{\mathrm{v}} w\right\|_{L^{2}}^{1-\varepsilon}\left\|\nabla_{h} \triangle_{q}^{\mathrm{v}} w\right\|_{L^{2}}^{\varepsilon} .
\end{aligned}
$$

En tenant compte de l'inégalité

$$
\left\|S_{q^{\prime}-1}^{\mathrm{v}} u\right\|_{L_{\mathrm{v}}^{\infty} L_{h}^{2}}^{\varepsilon}\left\|S_{q^{\prime}-1}^{\mathrm{v}} \nabla_{h} u\right\|_{L_{\mathrm{v}}^{\infty} L_{h}^{2}}^{1-\varepsilon} \leq C \sqrt{q^{\prime}}\|u\|_{H^{0,1 / 2}}^{\varepsilon}\left\|\nabla_{h} u\right\|_{H^{0,1 / 2}}^{1-\varepsilon},
$$

on obtient

$$
\begin{aligned}
T_{2, N}^{h} & \leq \sum_{q \leq N} 2^{-q} C \sqrt{\frac{q}{\varepsilon}}\|u\|_{H^{0,1 / 2}}^{\varepsilon}\left\|\nabla_{h} u\right\|_{H^{0,1 / 2}}^{1-\varepsilon}\left\|\nabla_{h} \triangle_{q^{\prime}}^{\mathrm{v}} w\right\|_{L^{2}}\left\|\triangle_{q}^{\mathrm{v}} w\right\|_{L^{2}}^{1-\varepsilon}\left\|\nabla_{h} \triangle_{q}^{\mathrm{v}} w\right\|_{L^{2}}^{\varepsilon} \\
& \leq C \sqrt{\frac{N}{\varepsilon}}\|u\|_{H^{0,1 / 2}}^{\varepsilon}\left\|\nabla_{h} u\right\|_{H^{0,1 / 2}}^{1-\varepsilon}\|w\|_{H^{0,-1 / 2}}^{1-\varepsilon}\left\|\nabla_{h} w\right\|_{H^{0,-1 / 2}}^{1+\varepsilon} \sum_{q} b_{q}
\end{aligned}
$$

où $b_{q} \in \ell^{1}(\mathbb{N})$. En utilisant maintenant l'inégalité $a b \leq a^{\frac{2}{1-\varepsilon}}+b^{\frac{2}{1+\varepsilon}}$, on a

$$
T_{2, N}^{h} \leq\left(\frac{C N}{\varepsilon}\right)^{\frac{1}{1-\varepsilon}}\|u\|_{H^{0,1 / 2}}^{\frac{2 \varepsilon}{1-\varepsilon}}\left\|\nabla_{h} u\right\|_{H^{0,1 / 2}}^{2}\|w\|_{H^{0,-1 / 2}}^{2}+\frac{\nu_{h}}{100}\left\|\nabla_{h} w\right\|_{H^{0,-1 / 2}}^{2} .
$$

Soit $N \geq 3$. Choisissons $\varepsilon=\frac{1}{1+\ln N} \in(0,1 / 2)$. Alors on a

$$
T_{2, N}^{h} \leq(C N(1+\ln N))^{\left(1+\frac{1}{\ln N}\right)} f(t)\|w\|_{H^{0,-1 / 2}}^{2}+\frac{\nu_{h}}{100}\left\|\nabla_{h} w\right\|_{H^{0,-1 / 2}}^{2} .
$$

Comme $(1+x)^{1 / x} \leq e$ et comme $N^{\frac{1}{\ln N}}=e$, on obtient finalement

$$
T_{2, N}^{h} \leq C N(1+\ln N) f(t)\|w\|_{H^{0,-1 / 2}}^{2}+\frac{\nu_{h}}{50}\left\|\nabla_{h} w\right\|_{H^{0,-1 / 2}}^{2}
$$


En prenant $N=1-\ln \|w\|_{H^{0,-1 / 2}}^{2}$, on obtient

$$
\begin{gathered}
T_{2}^{h} \leq C f(t)\|w\|_{H^{0,-1 / 2}}^{2}\left(1-\ln \|w\|_{H^{0,-1 / 2}}^{2}\right)\left(1+\ln \left(1-\ln \|w\|_{H^{0,-1 / 2}}^{2}\right)\right) \\
+\frac{\nu_{h}}{50}\left\|\nabla_{h} w\right\|_{H^{0,-1 / 2}}^{2} .
\end{gathered}
$$

Pour le dernier terme, qui contient le terme de reste, on utilise la même méthode. Soit $N$ fixé à la fin, on pose

$$
\begin{array}{r}
R_{N}^{h}=\sum_{q \leq N} 2^{-q} \int \sum_{\substack{i \in\{0, \pm 1\} \\
q^{\prime}>q-4}} \triangle_{q}^{\mathrm{v}}\left(\triangle_{q^{\prime}}^{\mathrm{v}} u \cdot \nabla_{h} \triangle_{q^{\prime}+i}^{\mathrm{v}} w\right) \triangle_{q}^{\mathrm{v}} w d x \quad \text { et } \\
R^{h, N}=\sum_{q \geq N} 2^{-q} \int \sum_{\substack{i \in\{0, \pm 1\} \\
q^{\prime}>q-4}} \triangle_{q}^{\mathrm{v}}\left(\triangle_{q^{\prime}}^{\mathrm{v}} u \nabla_{h} \triangle_{q^{\prime}+i}^{\mathrm{v}} w\right) \triangle_{q}^{\mathrm{v}} w d x .
\end{array}
$$

Pour $R_{N}^{h}$, on a par l'inégalité de Hölder

$$
\begin{aligned}
& R_{N}^{h} \leq \sum_{q \leq N} 2^{-q} \sum_{\substack{i \in\{0, \pm 1\} \\
q^{\prime}>q-4}}\left\|\triangle_{q}^{\mathrm{v}}\left(\triangle_{q^{\prime}}^{\mathrm{v}} u \nabla_{h} \triangle_{q^{\prime}+i}^{\mathrm{v}} w\right)\right\|_{L_{\mathrm{v}}^{1} L_{h}^{1+\varepsilon}}\left\|\triangle_{q}^{\mathrm{v}} w\right\|_{L_{\mathrm{v}}^{\infty} L_{h}^{\frac{2}{1-\varepsilon}}} \\
& \leq \sum_{q \leq N} 2^{-q} \sum_{\substack{i \in\{0, \pm 1\} \\
q^{\prime}>q-4}}\left\|\triangle_{q^{\prime}}^{\mathrm{v}} u\right\|_{L_{\mathrm{v}}^{2} L_{h}^{\frac{2}{\varepsilon}}}\left\|\nabla_{h} \triangle_{q^{\prime}+i}^{\mathrm{v}} w\right\|_{L^{2}}\left\|\triangle_{q}^{\mathrm{v}} w\right\|_{L_{\mathrm{v}}^{\infty} L_{h}^{1-\varepsilon}}
\end{aligned}
$$

Le lemme 4.3 nous donne

$$
\begin{aligned}
& R_{N}^{h} \leq \sum_{q \leq N} 2^{-q} \frac{C}{\sqrt{\varepsilon}} \sum_{\substack{i \in\{0, \pm 1\} \\
q^{\prime}>q-4}}\left\|\triangle_{q^{\prime}}^{\mathrm{v}} u\right\|_{L^{2}}^{\varepsilon}\left\|\nabla_{h} \triangle_{q^{\prime}}^{\mathrm{v}} u\right\|_{L^{2}}^{1-\varepsilon} \times \\
& \quad \times\left\|\nabla_{h} \triangle_{q^{\prime}+i}^{\mathrm{v}} w\right\|_{L^{2}}\left\|\triangle_{q}^{\mathrm{v}} w\right\|_{L_{\mathrm{v}}^{\infty} L_{h}^{2}}^{1-\varepsilon}\left\|\nabla_{h} \triangle_{q}^{\mathrm{v}} w\right\|_{L_{\mathrm{v}}^{\infty} L_{h}^{2}}^{\varepsilon} \\
& \leq \sum_{q \leq N} \frac{C}{\sqrt{\varepsilon}} \tilde{c}_{q} \sum_{q^{\prime} \geq q-1} b_{q^{\prime}}\|u\|_{H^{0,1 / 2}}^{\varepsilon}\left\|\nabla_{h} u\right\|_{H^{0,1 / 2}}^{1-\varepsilon}\|w\|_{H^{0,-1 / 2}}^{1-\varepsilon}\left\|\nabla_{h} w\right\|_{H^{0,-1 / 2}}^{1+\varepsilon}
\end{aligned}
$$

avec $b_{q^{\prime}} \in \ell^{1}(\mathbb{N})$ et $\tilde{c}_{q} \in l^{2}(\mathbb{N})$. On a donc

$$
\sum_{0 \leq q \leq N} \tilde{c}_{q} \leq \sqrt{N}\left(\sum_{0 \leq q \leq N} \tilde{c}_{q}^{2}\right)^{1 / 2} \leq \sqrt{N} .
$$

Alors

$$
\begin{aligned}
R_{N}^{h} & \leq C \sqrt{\frac{N}{\varepsilon}}\|u\|_{H^{0,1 / 2}}^{\varepsilon}\left\|\nabla_{h} u\right\|_{H^{0,1 / 2}}^{1-\varepsilon}\|w\|_{H^{0,-1 / 2}}^{1-\varepsilon}\left\|\nabla_{h} w\right\|_{H^{0,-1 / 2}}^{1+\varepsilon} \\
& \leq\left(C \frac{N}{\varepsilon}\right)^{\frac{1}{1-\varepsilon}}\|u\|_{H^{0,1 / 2}}^{\frac{\varepsilon}{1-\varepsilon}}\left\|\nabla_{h} u\right\|_{H^{0,1 / 2}}^{2}\|w\|_{H^{0,-1 / 2}}^{2}+\frac{\nu_{h}}{100}\left\|\nabla_{h} w\right\|_{H^{0,-1 / 2}}^{2} .
\end{aligned}
$$


Exactement comme dans l'estimation pour $T_{2}^{h, N}$, on obtient facilement

$$
R^{h, N} \leq 2^{-2 N / 3}\|u\|_{H^{0,1 / 2}}^{2 / 3}\left\|\nabla_{h} u\right\|_{H^{0,1 / 2}}^{2}\|w\|_{H^{0,-1 / 2}}^{2 / 3}+\frac{\nu_{h}}{100}\left\|\nabla_{h} w\right\|_{H^{0,-1 / 2}}^{2}
$$

Donc, pour $R^{h}$ on a les mêmes estimations que pour $T_{2}^{h}$.

Estimons maintenant les autres termes horizontaux (ne contenant que des dérivées horizontale). On a la décomposition

$$
\sum_{q \geq-1} 2^{-q} \sum_{\left|q-q^{\prime}\right| \leq 4} \int \triangle_{q}^{\mathrm{v}}\left(w^{h} \nabla_{h} v\right) \triangle_{q}^{\mathrm{v}} w d x=\tilde{T}_{1}^{h}+\tilde{T}_{2}^{h}+\tilde{R}^{h}
$$

où on a noté

$$
\begin{aligned}
& \tilde{T}_{1}^{h}=\sum_{q} 2^{-q} \sum_{\left|q-q^{\prime}\right| \leq 4} \int \triangle_{q}^{\mathrm{v}}\left(S_{q^{\prime}-1}^{\mathrm{v}} w_{h} \nabla_{h} \triangle_{q^{\prime}}^{\mathrm{v}} v\right) \triangle_{q}^{\mathrm{v}} w d x \\
& \tilde{T}_{2}^{h}=\sum_{q} 2^{-q} \sum_{\left|q-q^{\prime}\right| \leq 4} \int \triangle_{q}^{\mathrm{v}}\left(S_{q^{\prime}-1}^{\mathrm{v}} \nabla_{h} v \triangle_{q^{\prime}}^{\mathrm{v}} w\right) \triangle_{q}^{\mathrm{v}} w d x \text { et } \\
& \tilde{R}^{h}=\sum_{q} 2^{-q} \sum_{\substack{i \in\{0, \pm 1\} \\
q^{\prime}>q-4}} \int \triangle_{q}\left(\triangle_{q^{\prime}}^{\mathrm{v}} w^{h} \nabla^{h} \triangle_{q^{\prime}+i}^{\mathrm{v}} v\right) \triangle_{q}^{\mathrm{v}} w d x .
\end{aligned}
$$

Pour estimer $\tilde{T}_{1}^{h}$, on écrit

$$
\tilde{T}_{1}^{h} \leq \sum_{q \geq-1} 2^{-q} \sum_{\left|q-q^{\prime}\right| \leq 4}\left\|S_{q^{\prime}-1}^{\mathrm{v}} w^{h}\right\|_{L_{\mathrm{v}}^{\infty} L_{h}^{4}}\left\|\triangle_{q^{\prime}}^{\mathrm{v}} \nabla_{h} v\right\|_{L^{2}}\left\|\triangle_{q}^{\mathrm{v}} w\right\|_{L_{\mathrm{v}}^{2} L_{h}^{4}}
$$

On utilise maintenant l'inégalité

$$
\begin{aligned}
\left\|S_{q}^{\mathrm{v}} w_{h}\right\|_{L_{\mathrm{v}}^{\infty} L_{h}^{4}} & \leq \sum_{j \leq q} 2^{j / 2}\left\|\triangle_{j}^{\mathrm{v}} w_{h}\right\|_{L_{\mathrm{v}}^{2} L_{h}^{4}} \\
& \leq \sum_{j \leq q} 2^{j / 2}\left\|\triangle_{j}^{\mathrm{v}} w\right\|_{L^{2}}^{1 / 2}\left\|\triangle_{j}^{\mathrm{v}} \nabla_{h} w\right\|_{L^{2}}^{1 / 2} \\
& \leq C 2^{q}\|w\|_{H^{0,-1 / 2}}^{1 / 2}\left\|\nabla_{h} w\right\|_{H^{0,-1 / 2}}^{1 / 2} .
\end{aligned}
$$

On obtient ainsi

$\tilde{T}_{1}^{h} \leq C \sum_{q} \sum_{\left|q-q^{\prime}\right| \leq 4}\|w\|_{H^{0,-1 / 2}}^{1 / 2}\left\|\nabla_{h} w\right\|_{H^{0,-1 / 2}}^{1 / 2}\left\|\nabla_{h} \triangle_{q^{\prime}}^{\mathrm{v}} v\right\|_{L^{2}}\left\|\triangle_{q}^{\mathrm{v}} w\right\|_{L^{2}}^{1 / 2}\left\|\nabla_{h} \triangle_{q}^{\mathrm{v}} w\right\|_{L^{2}}^{1 / 2}$

Donc, on a l'estimation

$$
\begin{aligned}
\tilde{T}_{1}^{h} & \leq C\|w\|_{H^{0,-1 / 2}}\left\|\nabla_{h} w\right\|_{H^{0,-1 / 2}}\left\|\nabla_{h} v\right\|_{H^{0,1 / 2}} \\
& \leq C\|w\|_{H^{0,-1 / 2}}^{2}\left\|\nabla_{h} v\right\|_{H^{0,1 / 2}}^{2}+\frac{\nu_{h}}{100}\left\|\nabla_{h} w\right\|_{H^{0,-1 / 2}}^{2} .
\end{aligned}
$$


Pour $\tilde{T}_{2}^{h}$, on a

$$
\tilde{T}_{2}^{h} \leq \sum_{q \geq-1} 2^{-q} \sum_{\left|q-q^{\prime}\right| \leq 4}\left\|S_{q^{\prime}-1}^{\mathrm{v}} \nabla_{h} v\right\|_{L_{\mathrm{v}}^{\infty} L_{h}^{2}}\left\|\triangle_{q^{\prime}}^{\mathrm{v}} w_{h}\right\|_{L_{\mathrm{v}}^{2} L_{h}^{4}}\left\|\triangle_{q}^{\mathrm{v}} w\right\|_{L_{\mathrm{v}}^{2} L_{h}^{4}} .
$$

En utilisant le lemme 3.2, on a

$$
\begin{aligned}
\tilde{T}_{2}^{h} \leq \sum_{q \geq-1} 2^{-q} \sum_{\left|q-q^{\prime}\right| \leq 4} \| & S_{q^{\prime}-1}^{\mathrm{v}} \nabla_{h} v\left\|_{L_{\mathrm{v}}^{\infty} L_{h}^{2}}\right\| \triangle_{q^{\prime}}^{\mathrm{v}} w_{h} \|_{L^{2}}^{1 / 2} \\
& \times\left\|\triangle_{q^{\prime}}^{\mathrm{v}} \nabla_{h} w_{h}\right\|_{L^{2}}^{1 / 2}\left\|\triangle_{q}^{\mathrm{v}} w\right\|_{L^{2}}^{1 / 2}\left\|\nabla_{h} \triangle_{q}^{\mathrm{v}} w\right\|_{L^{2}}^{1 / 2} .
\end{aligned}
$$

En tenant compte du fait que

$$
\left\|S_{q^{\prime}-1}^{\mathrm{v}} \nabla_{h} v\right\|_{L_{\mathrm{v}}^{\infty} L_{h}^{2}} \leq C \sqrt{q^{\prime}}\left\|\nabla_{h} v\right\|_{H^{0,1 / 2}}
$$

on obtient

$$
\begin{aligned}
\tilde{T}_{2}^{h} \leq \sum_{q \geq-1} 2^{-q} \sum_{\left|q-q^{\prime}\right| \leq 4} C & \sqrt{q^{\prime}}\left\|\nabla_{h} v\right\|_{H^{0,1 / 2}}\left\|\triangle_{q^{\prime}}^{\mathrm{v}} w\right\|_{L^{2}}^{1 / 2} \times \\
& \times\left\|\nabla_{h} \triangle_{q^{\prime}}^{\mathrm{v}} w\right\|_{L^{2}}^{1 / 2}\left\|\triangle_{q}^{\mathrm{v}} w\right\|_{L^{2}}^{1 / 2}\left\|\nabla_{h} \triangle_{q}^{\mathrm{v}} w\right\|_{L^{2}}^{1 / 2} .
\end{aligned}
$$

Soit maintenant $N$ un paramètre entier qui sera choisi à la fin. On décompose

$$
\tilde{T}_{2}^{h}=\tilde{T}_{2, N}^{h}+\tilde{T}_{2}^{h, N},
$$

où on a noté

$$
\begin{gathered}
\tilde{T}_{2, N}^{h}=C \sum_{q \leq N} 2^{-q} \sum_{\left|q-q^{\prime}\right| \leq 4} \sqrt{q^{\prime}}\left\|\nabla_{h} v\right\|_{H^{0,1 / 2}}\left\|\triangle_{q^{\prime}}^{\mathrm{v}} w\right\|_{L^{2}}^{1 / 2}\left\|\nabla_{h} \triangle_{q^{\prime}}^{\mathrm{v}} w\right\|_{L^{2}}^{1 / 2} \times \\
\times\left\|\triangle_{q}^{\mathrm{v}} w\right\|_{L^{2}}^{1 / 2}\left\|\nabla_{h} \triangle_{q}^{\mathrm{v}} w\right\|_{L^{2}}^{1 / 2}
\end{gathered}
$$

et

$$
\begin{gathered}
\tilde{T}_{2}^{h, N}=C \sum_{q \geq N} 2^{-q} \sum_{\left|q-q^{\prime}\right| \leq 4} \sqrt{q^{\prime}}\left\|\nabla_{h} v\right\|_{H^{0,1 / 2}}\left\|\triangle_{q^{\prime}}^{\mathrm{v}} w\right\|_{L^{2}}^{1 / 2}\left\|\nabla_{h} \triangle_{q^{\prime}}^{\mathrm{v}} w\right\|_{L^{2}}^{1 / 2} \times \\
\times\left\|\triangle_{q}^{\mathrm{v}} w\right\|_{L^{2}}^{1 / 2}\left\|\nabla_{h} \triangle_{q}^{\mathrm{v}} w\right\|_{L^{2}}^{1 / 2} .
\end{gathered}
$$

On va estimer différemment la partie "basses fréquences" et la partie "hautes fréquences". Pour la première partie, on tient compte du fait qu'on estime $w$ dans l'espace $H^{0,-1 / 2}$. On a ainsi l'estimation

$$
\begin{aligned}
\tilde{T}_{2, N}^{h} \leq C \sqrt{N}\left\|\nabla_{h} v\right\|_{H^{0,1 / 2}} \sum_{q \geq-1} 2^{-q} \sum_{\left|q-q^{\prime}\right| \leq 4}\left\|\triangle_{q^{\prime}}^{\mathrm{v}} w\right\|_{L^{2}}^{1 / 2} \times \\
\\
\times\left\|\nabla_{h} \triangle_{q^{\prime}}^{\mathrm{v}} w\right\|_{L^{2}}^{1 / 2}\left\|\triangle_{q}^{\mathrm{v}} w\right\|_{L^{2}}^{1 / 2}\left\|\nabla_{h} \triangle_{q}^{\mathrm{v}} w\right\|_{L^{2}}^{1 / 2} \\
\leq C \sqrt{N}\left\|\nabla_{h} v\right\|_{H^{0,1 / 2}}\|w\|_{H^{0,-1 / 2}}\left\|\nabla_{h} w\right\|_{H^{0,-1 / 2}} \sum_{q \geq-1} \tilde{b}_{q},
\end{aligned}
$$

où la suite $\tilde{b}_{q}$ est sommable et elle est donnée par $\tilde{b}_{q}=c_{q} \sum_{\left|q-q^{\prime}\right| \leq 4} c_{q^{\prime}}$, avec $c_{q}$ suite de carré sommable, définie par $c_{q}\|w\|_{H^{0,-1 / 2}}=2^{-q / 2}\left\|\triangle_{q}^{\mathrm{v}} w\right\|_{L^{2}}$. 
Pour la somme "hautes fréquences", on utilise le fait que $w$ appartient à l'espace $H^{0,1 / 2}$, et donc, on a

$$
\sum_{\left|q-q^{\prime}\right| \leq 4}\left\|\triangle_{q^{\prime}}^{\mathrm{v}} w\right\|_{L^{2}}^{1 / 2}\left\|\triangle_{q^{\prime}}^{\mathrm{v}} \nabla_{h} w\right\|_{L^{2}}^{1 / 2} \leq 2^{-q / 2} c_{q}\|w\|_{H^{0,1 / 2}}^{1 / 2}\left\|\nabla_{h} w\right\|_{H^{0,1 / 2}}^{1 / 2}
$$

D'autre part, vu qu'on estime $w$ dans l'espace $H^{0,-1 / 2}$, on a

$$
\left\|\triangle_{q}^{\mathrm{v}} w\right\|_{L^{2}}^{1 / 2}\left\|\triangle_{q}^{\mathrm{v}} \nabla_{h} w\right\|_{L^{2}}^{1 / 2} \leq 2^{q / 2} \tilde{c}_{q}\|w\|_{H^{0,-1 / 2}}^{1 / 2}\left\|\nabla_{h} w\right\|_{H^{0,-1 / 2}}^{1 / 2}
$$

En conséquence, on a

$$
\begin{aligned}
& \tilde{T}_{2}^{h, N}=C \sum_{q \geq N} 2^{-q} \sum_{\left|q-q^{\prime}\right| \leq 4} \sqrt{q^{\prime}}\left\|\nabla_{h} v\right\|_{H^{0,1 / 2}}\left\|\triangle_{q^{\prime}}^{\mathrm{v}} w\right\|_{L^{2}}^{1 / 2} \times \\
& \times\left\|\nabla_{h} \triangle_{q^{\prime}}^{\mathrm{v}} w\right\|_{L^{2}}^{1 / 2}\left\|\triangle_{q}^{\mathrm{v}} w\right\|_{L^{2}}^{1 / 2}\left\|\nabla_{h} \triangle_{q}^{\mathrm{v}} w\right\|_{L^{2}}^{1 / 2} \\
& \leq C \sum_{q \geq N} 2^{-q} \sqrt{q} c_{q} \tilde{c}_{q}\left\|\nabla_{h} v\right\|_{H^{0,1 / 2}}\|w\|_{H^{0,1 / 2}}^{1 / 2}\left\|\nabla_{h} w\right\|_{H^{0,1 / 2}}^{1 / 2}\|w\|_{H^{0,-1 / 2}}^{1 / 2}\left\|\nabla_{h} w\right\|_{H^{0,-1 / 2}}^{1 / 2}
\end{aligned}
$$

Vu que $2^{-q / 2} \sqrt{q} \leq 1$, et en tenant compte du fait que $\sum_{q} c_{q} \tilde{c}_{q} \leq 1$, on obtient

$$
\tilde{T}_{2}^{h, N} \leq C 2^{-N / 2}\left\|\nabla_{h} v\right\|_{H^{0,1 / 2}}\|w\|_{H^{0,1 / 2}}^{1 / 2}\left\|\nabla_{h} w\right\|_{H^{0,1 / 2}}^{1 / 2}\|w\|_{H^{0,-1 / 2}}^{1 / 2}\left\|\nabla_{h} w\right\|_{H^{0,-1 / 2}}^{1 / 2} .
$$

On a obtenu finalement l'estimation

$$
\begin{aligned}
\tilde{T}_{2}^{h} \leq & C \sqrt{N}\left\|\nabla_{h} v\right\|_{H^{0,1 / 2}}\|w\|_{H^{0,-1 / 2}}\left\|\nabla_{h} w\right\|_{H^{0,-1 / 2}} \\
& +C 2^{-N / 2}\left\|\nabla_{h} v\right\|_{H^{0,1 / 2}}\|w\|_{H^{0,1 / 2}}^{1 / 2}\left\|\nabla_{h} w\right\|_{H^{0,1 / 2}}^{1 / 2}\|w\|_{H^{0,-1 / 2}}^{1 / 2}\left\|\nabla_{h} w\right\|_{H^{0,-1 / 2}}^{1 / 2} .
\end{aligned}
$$

Donc, on a

$$
\begin{aligned}
\tilde{T}_{2}^{h} \leq C N\left\|\nabla_{h} v\right\|_{H^{0,1 / 2}}^{2}\|w\|_{H^{0,-1 / 2}}^{2}+2^{-2 N / 3}\left\|\nabla_{h} v\right\|_{H^{0,1 / 2}}^{4 / 3}\|w\|_{H^{0,1 / 2}}^{2 / 3} \times \\
\times\left\|\nabla_{h} w\right\|_{H^{0,1 / 2}}^{2 / 3}\|w\|_{H^{0,-1 / 2}}^{2 / 3}+\frac{\nu_{h}}{50}\left\|\nabla_{h} w\right\|_{H^{0,-1 / 2}}^{2}
\end{aligned}
$$

En choisissant $N$ tel que

$$
2^{-2 N / 3}\|w(t)\|_{H^{0,-1 / 2}}^{2 / 3}=\|w(t)\|_{H^{0,-1 / 2}}^{2},
$$

ce qui revient au $N=-\ln \|w\|_{H^{0,-1 / 2}}^{2}$, on obtient finalement

$$
\tilde{T}_{2}^{h} \leq C f(t)\left(1-\ln \|w(t)\|_{H^{0,-1 / 2}}^{2}\right)\|w(t)\|_{H^{0,-1 / 2}}^{2}+\frac{\nu_{h}}{50}\left\|\nabla_{h} w\right\|_{H^{0,-1 / 2}}^{2}
$$


Afin d'estimer le terme de reste $\tilde{R}^{h}$, on l'écrit $\tilde{R}^{h}=\tilde{R}_{N}^{h}+\tilde{R}^{h, N}$, avec

$$
\begin{aligned}
\tilde{R}_{N}^{h} & =\sum_{q \leq N} 2^{-q} \sum_{\substack{i \in\{0, \pm 1\} \\
q^{\prime}>q-4}} \int \triangle_{q}^{\mathrm{v}}\left(\triangle_{q^{\prime}}^{\mathrm{v}} w^{h} \nabla_{h} \triangle_{q^{\prime}+i}^{\mathrm{v}} v\right) \triangle_{q}^{\mathrm{v}} w d x \quad \text { et } \\
\tilde{R}^{h, N} & =\sum_{q \geq N} 2^{-q} \sum_{\substack{i \in\{0, \pm 1\} \\
q^{\prime}>q-4}} \int \triangle_{q}^{\mathrm{v}}\left(\triangle_{q^{\prime}}^{\mathrm{v}} w^{h} \nabla_{h} \triangle_{q^{\prime}+i}^{\mathrm{v}} v\right) \triangle_{q}^{\mathrm{v}} w d x
\end{aligned}
$$

Pour $\tilde{R}_{N}^{h}$, on a l'estimation

$$
\tilde{R}_{N}^{h} \leq \sum_{q \leq N} 2^{-q} \sum_{\substack{i \in\{0, \pm 1\} \\ q^{\prime}>q-4}}\left\|\triangle_{q^{\prime}}^{\mathrm{v}} w\right\|_{L_{\mathrm{v}}^{2} L_{h}^{4}}\left\|\nabla_{h} \triangle_{q^{\prime}}^{\mathrm{v}} v\right\|_{L^{2}}\left\|\triangle_{q}^{\mathrm{v}} w\right\|_{L_{\mathrm{v}}^{\infty} L_{h}^{4}} .
$$

En utilisant le lemme 3.3, on a

$$
\left\|\triangle_{q}^{\mathrm{v}} w\right\|_{L_{\mathrm{v}}^{\infty} L_{h}^{4}} \leq C 2^{q / 2}\left\|\triangle_{q}^{\mathrm{v}} w\right\|_{L_{\mathrm{v}}^{2} L_{h}^{4}} \leq C 2^{q / 2}\left\|\triangle_{q}^{\mathrm{v}} w\right\|_{L^{2}}^{1 / 2}\left\|\triangle_{q}^{\mathrm{v}} \nabla_{h} w\right\|_{L^{2}}^{1 / 2} .
$$

Vu que $w$ est dans l'espace $H^{0,-1 / 2}$, on a donc

$$
\left\|\triangle_{q}^{\mathrm{v}} w\right\|_{L_{\mathrm{v}}^{\infty} L_{h}^{4}} \leq C 2^{q} c_{q}\|w\|_{H^{0,-1 / 2}}^{1 / 2}\left\|\nabla_{h} w\right\|_{H^{0,-1 / 2}}^{1 / 2}
$$

$\operatorname{avec} c_{q} \in l^{2}(\mathbb{N})$.

De la même manière, on a

$$
\left\|\triangle_{q^{\prime}}^{\mathrm{v}} w\right\|_{L_{\mathrm{v}}^{2} L_{h}^{4}} \leq\left\|\triangle_{q^{\prime}}^{\mathrm{v}} w\right\|_{L^{2}}^{1 / 2}\left\|\nabla_{h} \triangle_{q^{\prime}}^{\mathrm{v}} w\right\|_{L^{2}}^{1 / 2} \leq C 2^{q^{\prime} / 2} c_{q^{\prime}}\|w\|_{H^{0,-1 / 2}}^{1 / 2}\left\|\nabla_{h} w\right\|_{H^{0,-1 / 2}}^{1 / 2},
$$
avec $c_{q^{\prime}} \in l^{2}(\mathbb{N})$.

Comme $v$ appartient à l'espace $H^{0,1 / 2}$, on a

$$
\left\|\nabla_{h} \triangle_{q^{\prime}}^{\mathrm{v}} v\right\|_{L^{2}} \leq 2^{-q^{\prime} / 2} \tilde{c}_{q^{\prime}}\left\|\nabla_{h} v\right\|_{H^{0,1 / 2}} \quad \text { avec } \quad \tilde{c}_{q^{\prime}} \in l^{2}(\mathbb{N}) .
$$

On obtient ainsi

$$
\tilde{R}_{N}^{h} \leq C \sum_{q \leq N} c_{q} \sum_{\substack{i \in\{0, \pm 1\} \\ q^{\prime}>q-4}} c_{q^{\prime}} \tilde{c}_{q^{\prime}}\left\|\nabla_{h} v\right\|_{H^{0,1 / 2}}\|w\|_{H^{0,-1 / 2}}\left\|\nabla_{h} w\right\|_{H^{0,-1 / 2}} .
$$

Comme

$$
\sum_{q^{\prime} \geq-1} c_{q} c_{q^{\prime}}=1 \quad \text { et comme } \quad \sum_{q \leq N} c_{q} \leq \sqrt{N}\left(\sum_{q} c_{q}^{2}\right)^{1 / 2} \leq \sqrt{N}
$$

on obtient

$$
\begin{aligned}
\tilde{R}_{N}^{h} & \leq C \sqrt{N}\left\|\nabla_{h} v\right\|_{H^{0,1 / 2}}\|w\|_{H^{0,-1 / 2}}\left\|\nabla_{h} w\right\|_{H^{0,-1 / 2}} \\
& \leq C N\left\|\nabla_{h} v\right\|_{H^{0,1 / 2}}^{2}\|w\|_{H^{0,-1 / 2}}^{2}+\frac{\nu_{h}}{100}\left\|\nabla_{h} w\right\|_{H^{0,-1 / 2}}^{2}
\end{aligned}
$$


Pour estimer $\tilde{R}^{h, N}$, on écrit

$$
\begin{gathered}
\tilde{R}^{h, N} \leq \sum_{q \geq N} 2^{-q} \sum_{\substack{i \in\{0, \pm 1\} \\
q^{\prime}>q-4}}\left\|\triangle_{q^{\prime}}^{\mathrm{v}} w\right\|_{L_{\mathrm{v}}^{2} L_{h}^{4}}\left\|\triangle_{q^{\prime}+i}^{\mathrm{v}} \nabla_{h} v\right\|_{L^{2}}\left\|\triangle_{q}^{\mathrm{v}} w\right\|_{L_{\mathrm{v}}^{\infty} L_{h}^{4}} \\
\leq \sum_{q \geq N} 2^{-q / 2} \sum_{\substack{i \in\{0, \pm 1\} \\
q^{\prime}>q-4}}\left\|\triangle_{q^{\prime}}^{\mathrm{v}} w\right\|_{L^{2}}^{1 / 2}\left\|\nabla_{h} \triangle_{q^{\prime}}^{\mathrm{v}} w\right\|_{L^{2}}^{1 / 2}\left\|\nabla_{h} \triangle_{q^{\prime}+i}^{\mathrm{v}} v\right\|_{L^{2}} \times \\
\times\left\|\triangle_{q}^{\mathrm{v}} w\right\|_{L^{2}}^{1 / 2}\left\|\triangle_{q}^{\mathrm{v}} \nabla_{h} w\right\|_{L^{2}}^{1 / 2} .
\end{gathered}
$$

On a l'inégalité

$$
\left\|\triangle_{q^{\prime}}^{\mathrm{v}} w\right\|_{L^{2}}^{1 / 2}\left\|\triangle_{q^{\prime}}^{\mathrm{v}} \nabla_{h} w\right\|_{L^{2}}^{1 / 2} \leq C 2^{-q^{\prime} / 2} c_{q^{\prime}}\|w\|_{H^{0,1 / 2}}^{1 / 2}\left\|\nabla_{h} w\right\|_{H^{0,1 / 2}}^{1 / 2}
$$

avec $c_{q^{\prime}}$ suite de carré sommable. On a aussi les estimations suivantes

$$
\begin{gathered}
\left\|\nabla_{h} \triangle_{q^{\prime}}^{\mathrm{v}} v\right\|_{L^{2}} \leq 2^{-q^{\prime} / 2} c_{q^{\prime}}\left\|\nabla_{h} v\right\|_{H^{0,1 / 2}} \quad \text { et } \\
\left\|\triangle_{q}^{\mathrm{v}} w\right\|_{L^{2}}^{1 / 2}\left\|\triangle_{q}^{\mathrm{v}} \nabla_{h} w\right\|_{L^{2}}^{1 / 2} \leq C 2^{q / 2} \tilde{c}_{q}\|w\|_{H^{0,-1 / 2}}^{1 / 2}\left\|\nabla_{h} w\right\|_{H^{0,-1 / 2}}^{1 / 2},
\end{gathered}
$$

où $\sum_{q} \tilde{c}_{q}^{2}=1$.

On obtient ainsi

$$
\begin{aligned}
& \tilde{R}^{h, N} \leq \sum_{q \geq N} \sum_{q^{\prime}>q-4} 2^{-q^{\prime}} c_{q^{\prime}}^{2}\|w\|_{H^{0,1 / 2}}^{1 / 2}\left\|\nabla_{h} w\right\|_{H^{0,1 / 2}}^{1 / 2} \times \\
& \quad \times\left\|\nabla_{h} v\right\|_{H^{0,1 / 2} \tilde{c}_{q}}\|w\|_{H^{0,-1 / 2}}^{1 / 2}\left\|\nabla_{h} w\right\|_{H^{0,-1 / 2}}^{1 / 2} \\
& \leq 2^{-N}\|w\|_{H^{0,-1 / 2}}^{1 / 2}\left\|\nabla_{h} w\right\|_{H^{0,-1 / 2}}^{1 / 2}\left\|\nabla_{h} v\right\|_{H^{0,1 / 2}}\left\|\nabla_{h} w\right\|_{H^{0,1 / 2}}^{1 / 2}\|w\|_{H^{0,1 / 2}}^{1 / 2} \\
& \leq 2^{-4 N / 3}\|w\|_{H^{0,1 / 2}}^{2 / 3}\left\|\nabla_{h} w\right\|_{H^{0,1 / 2}}^{2 / 3}\left\|\nabla_{h} v\right\|_{H^{0,1 / 2}}^{4 / 3}\|w\|_{H^{0,-1 / 2}}^{2 / 3}+\frac{\nu_{h}}{100}\left\|\nabla_{h} w\right\|_{H^{0,-1 / 2}}^{2} .
\end{aligned}
$$

En sommant les estimations, on obtient

$$
\tilde{R}^{h} \leq C N\|w\|_{H^{0,-1 / 2}}^{2}+C 2^{-4 N / 3} f(t)\|w\|_{H^{0,-1 / 2}}^{2 / 3}+\frac{\nu_{h}}{50}\left\|\nabla_{h} w\right\|_{H^{0,-1 / 2}}^{2} .
$$

En choisissant $N$ tel que

$$
2^{-4 N / 3}\|w(t)\|_{H^{0,-1 / 2}}^{2 / 3}=\|w(t)\|_{H^{0,-1 / 2}}^{2},
$$

c'est-à-dire, à une constante près,

$$
N=-\ln \|w\|_{H^{0,-1 / 2}}^{2},
$$

on obtient à la fin

$$
\tilde{R}^{h} \leq C f(t)\left(1-\ln \|w\|_{H^{0,-1 / 2}}^{2}\right)\|w\|_{H^{0,-1 / 2}}^{2}+\frac{\nu_{h}}{50}\left\|\nabla_{h} w\right\|_{H^{0,-1 / 2}}^{2} .
$$


Étudions maintenant les termes contenant des dérivées verticales. Nous allons nous ramener à des termes ne contenant que des dérivées horizontales, en tenant toujours compte du fait que $\partial_{3} u^{3}=-\operatorname{div}_{h} u^{h}$. Il s'agit de deux termes suivants

$$
\begin{aligned}
& I=\sum_{q \geq-1} 2^{-q} \int \triangle_{q}^{\mathrm{v}}\left(u^{3} \partial_{3} w\right) \triangle_{q}^{\mathrm{v}} w d x \quad \text { et } \\
& \tilde{I}=\sum_{q \geq-1} 2^{-q} \int \triangle_{q}^{\mathrm{v}}\left(w_{3} \partial_{3} v\right) \triangle_{q}^{\mathrm{v}} w d x .
\end{aligned}
$$

Pour estimer le premier terme, on utilise la décomposition donnée dans [6] (voir la démonstration du lemme 3). On écrit ainsi la décomposition

$$
I=\sum_{j=1}^{4} I_{j}
$$

où

$$
\begin{aligned}
& I_{1}=\sum_{q \geq-1} 2^{-q} \int S_{q-1}^{\mathrm{v}} u^{3} \partial_{3} \triangle_{q}^{\mathrm{v}} w \triangle_{q}^{\mathrm{v}} w d x \\
& I_{2}=\sum_{q \geq-1} \sum_{\left|q-q^{\prime}\right| \leq 4} 2^{-q} \int\left(S_{q-1}^{\mathrm{v}} u^{3}-S_{q^{\prime}-1}^{\mathrm{v}} u^{3}\right) \partial_{3} \triangle_{q^{\prime}}^{\mathrm{v}} \triangle_{q}^{\mathrm{v}} w \triangle_{q}^{\mathrm{v}} w d x \\
& I_{3}=\sum_{q \geq-1} \sum_{\left|q-q^{\prime}\right| \leq 4} 2^{-q} \int\left[\triangle_{q}^{\mathrm{v}} ; S_{q^{\prime}-1}^{\mathrm{v}} u^{3}\right] \partial_{3} \triangle_{q^{\prime}}^{\mathrm{v}} w \triangle_{q}^{\mathrm{v}} w d x \\
& I_{4}=\sum_{q \geq-1} \sum_{q^{\prime} \geq q} 2^{-q} \int \triangle_{q}^{\mathrm{v}}\left(\triangle_{q^{\prime}}^{\mathrm{v}} u^{3} \partial_{3} \triangle_{q^{\prime}}^{\mathrm{v}} w\right) \triangle_{q}^{\mathrm{v}} w d x .
\end{aligned}
$$

Par une intégration par parties dans la variable verticale, on a

$$
\sum_{q \geq-1} 2^{-q} \int S_{q-1}^{\mathrm{v}} u^{3} \partial_{3} \triangle_{q}^{\mathrm{v}} w \triangle_{q}^{\mathrm{v}} w d x=\frac{1}{2} \sum_{q \geq-1} 2^{-q} \int S_{q-1}^{\mathrm{v}} \operatorname{div}{ }_{h} u^{h} \triangle_{q}^{\mathrm{v}} w \triangle_{q}^{\mathrm{v}} w d x
$$

De la même manière que pour le terme $\tilde{T}_{2}^{h}$, on obtient l'estimation

$$
\begin{gathered}
I_{1} \leq C \sqrt{N}\left\|\nabla_{h} u\right\|_{H^{0,1 / 2}}\|w\|_{H^{0,-1 / 2}}\left\|\nabla_{h} w\right\|_{H^{0,-1 / 2}} \\
\quad+2^{-N / 2}\|u\|_{H^{0,1 / 2}}^{1 / 2}\left\|\nabla_{h} u\right\|^{3 / 2}\|w\|_{H^{0,-1 / 2}}^{1 / 2}\left\|\nabla_{h} w\right\|_{H^{0,-1 / 2}}^{1 / 2} \\
\leq C N\left\|\nabla_{h} u\right\|_{H^{0,1 / 2}}^{2}\|w\|_{H^{0,-1 / 2}}^{2}+2^{-2 N / 3}\|u\|_{H^{0,1 / 2}}^{2 / 3}\left\|\nabla_{h} u\right\|_{H^{0,1 / 2}}^{2}\|w\|_{H^{0,-1 / 2}}^{2 / 3} \\
+\frac{\nu_{h}}{50}\left\|\nabla_{h} w\right\|_{H^{0,-1 / 2}}^{2} .
\end{gathered}
$$

Le choix $N=-\ln \|w\|_{H^{0,1 / 2}}^{2}$ nous donne l'inégalité

$$
I_{1} \leq C f(t)\left(1-\ln \|w(t)\|_{H^{0,1 / 2}}^{2}\right)\|w(t)\|_{H^{0,1 / 2}}^{2}+\frac{\nu_{h}}{50}\left\|\nabla_{h} w(t)\right\|_{H^{0,1 / 2}}^{2} .
$$


Pour $I_{2}$ on a exactement la même estimation. Afin d'estimer $I_{3}$, rappelons l'inégalité (3.8) du lemme 3.3, qui est bien connue

$$
\left\|\left[\triangle_{q}^{\mathrm{v}} ; a\right] b\right\|_{L_{\mathrm{v}}^{p} L_{h}^{r}} \leq C 2^{-q}\left\|\partial_{3} a\right\|_{L_{\mathrm{v}}^{p^{\prime}} L_{h}^{r^{\prime}}}\|b\|_{L_{\mathrm{v}}^{p^{\prime \prime}} L_{h}^{r^{\prime \prime}}}
$$

où on a $1 / p=1 / p^{\prime}+1 / p^{\prime \prime}$ et $1 / r=1 / r^{\prime}+1 / r^{\prime \prime}$. Dans notre cas, cela nous donne

$$
\begin{gathered}
I_{3} \leq \sum_{q \geq-1} 2^{-q} \sum_{\left|q-q^{\prime}\right| \leq 4}\left\|\left[\triangle_{q}^{\mathrm{v}} ; S_{q^{\prime}-1}^{\mathrm{v}} u^{3}\right] \partial_{3} \triangle_{q^{\prime}}^{\mathrm{v}} w\right\|_{L_{\mathrm{v}}^{2} L_{h}^{4 / 3}}\left\|\triangle_{q}^{\mathrm{v}} w\right\|_{L_{\mathrm{v}}^{2} L_{h}^{4}} \\
\leq \sum_{q \geq-1} C 2^{-q} \sum_{\left|q-q^{\prime}\right| \leq 4} 2^{-q^{\prime}}\left\|S_{q^{\prime}-1}^{\mathrm{v}} \operatorname{div}{ }_{h} u^{h}\right\|_{L_{\mathrm{v}}^{\infty} L_{h}^{2}}\left\|\partial_{3} \triangle_{q^{\prime}}^{\mathrm{v}} w\right\|_{L_{\mathrm{v}}^{2} L_{h}^{4}}\left\|\triangle_{q}^{\mathrm{v}} w\right\|_{L_{\mathrm{v}}^{2} L_{h}^{4}} \\
\leq \sum_{q \geq-1} C 2^{-q} \sum_{\left|q-q^{\prime}\right| \leq 4}\left\|S_{q^{\prime}-1}^{\mathrm{v}} \operatorname{div}{ }_{h} u^{h}\right\|_{L_{\mathrm{v}}^{\infty} L_{h}^{2}}\left\|\triangle_{q^{\prime}}^{\mathrm{v}} w\right\|_{L^{2}}^{1 / 2}\left\|\triangle_{q^{\prime}}^{\mathrm{v}} \nabla_{h} w\right\|_{L^{2}}^{1 / 2} \times \\
\times\left\|\triangle_{q}^{\mathrm{v}} w\right\|_{L^{2}}^{1 / 2}\left\|\triangle_{q}^{\mathrm{v}} \nabla_{h} w\right\|_{L^{2}}^{1 / 2} .
\end{gathered}
$$

On utilise les mêmes estimations que pour $\tilde{T}_{2}^{h}$, pour arriver finalement à l'inégalité

$$
I_{3} \leq C f(t)\left(1-\ln \|w(t)\|_{H^{0,-1 / 2}}^{2}\right)\|w(t)\|_{H^{0,-1 / 2}}^{2}+\frac{\nu_{h}}{50}\left\|\nabla_{h} w\right\|_{H^{0,-1 / 2}}^{2} .
$$

Pour $I_{4}$, on écrit d'abord l'estimation suivante

$$
\begin{gathered}
I_{4} \leq \sum_{q \geq-1} 2^{-q} \sum_{q^{\prime} \geq q-1}\left\|\triangle_{q^{\prime}}^{\mathrm{v}} u^{3}\right\|_{L^{2}}\left\|\triangle_{q^{\prime}}^{\mathrm{v}} \partial_{3} w\right\|_{L_{\mathrm{v}}^{2} L_{h}^{4}}\left\|\triangle_{q}^{\mathrm{v}} w\right\|_{L_{\mathrm{v}}^{2} L_{h}^{4}} \\
\leq C \sum_{q \geq-1} 2^{-q} \sum_{q^{\prime} \geq q-1} 2^{-q^{\prime}}\left\|\triangle_{q^{\prime}}^{\mathrm{v}} \partial_{3} u^{3}\right\|_{L^{2}}\left\|\triangle_{q^{\prime}}^{\mathrm{v}} \partial_{3} w\right\|_{L_{\mathrm{v}}^{2} L_{h}^{4}}\left\|\triangle_{q}^{\mathrm{v}} w\right\|_{L_{\mathrm{v}}^{2} L_{h}^{4}} \\
\leq C \sum_{q \geq-1} 2^{-q} \sum_{q^{\prime} \geq q-1}\left\|\triangle_{q^{\prime}}^{\mathrm{v}} \operatorname{div}{ }_{h} u^{h}\right\|_{L^{2}}\left\|\triangle_{q^{\prime}}^{\mathrm{v}} w\right\|_{L^{2}}^{1 / 2} \times \\
\times\left\|\triangle_{q^{\prime}}^{\mathrm{v}} \nabla_{h} w\right\|_{L^{2}}^{1 / 2}\left\|\triangle_{q}^{\mathrm{v}} w\right\|_{L^{2}}^{1 / 2}\left\|\triangle_{q}^{\mathrm{v}} \nabla_{h} w\right\|_{L^{2}}^{1 / 2} .
\end{gathered}
$$

D'ici, on fait comme pour le terme $\tilde{R}^{h}$ et on obtient à la fin, l'inégalité

$$
I_{4} \leq C\left(1-\ln \|w(t)\|_{H^{0,-1 / 2}}^{2}\right) f(t)\|w(t)\|_{H^{0,-1 / 2}}^{2}+\frac{\nu_{h}}{50}\left\|\nabla_{h} w(t)\right\|_{H^{0,-1 / 2}}^{2}
$$

Traitons maintenant le terme

$$
\tilde{I}=\sum_{q \geq-1} 2^{-q} \int \triangle_{q}^{\mathrm{v}}\left(w^{3} \partial_{3} u\right) \triangle_{q}^{\mathrm{v}} w d x .
$$


La décomposition de Bony nous donne $\tilde{I}=\tilde{T}_{1}^{\mathrm{v}}+\tilde{T}_{2}^{\mathrm{v}}+\tilde{R}^{\mathrm{v}}$, où

$$
\begin{aligned}
& \tilde{T}_{1}^{\mathrm{v}}=\sum_{q \geq-1} 2^{-q} \sum_{\left|q-q^{\prime}\right| \leq 4} \int \triangle_{q}^{\mathrm{v}}\left(S_{q^{\prime}-1}^{\mathrm{v}} w^{3} \partial_{3} \triangle_{q^{\prime}}^{\mathrm{v}} u\right) \triangle_{q}^{\mathrm{v}} w d x, \\
& \tilde{T}_{2}^{\mathrm{v}}=\sum_{q \geq-1} 2^{-q} \sum_{\left|q-q^{\prime}\right| \leq 4} \int \triangle_{q}^{\mathrm{v}}\left(S_{q^{\prime}-1}^{\mathrm{v}} \partial_{3} u \cdot \triangle_{q^{\prime}}^{\mathrm{v}} w^{3}\right) \triangle_{q}^{\mathrm{v}} w d x \quad \text { et } \\
& \tilde{R}^{\mathrm{v}}=\sum_{q \geq-1} 2^{-q} \sum_{q^{\prime} \geq q-1} \int \triangle_{q}^{\mathrm{v}}\left(\triangle_{q^{\prime}}^{\mathrm{v}} w^{3} \cdot \triangle_{q^{\prime}}^{\mathrm{v}} \partial_{3} u\right) \triangle_{q}^{\mathrm{v}} w d x .
\end{aligned}
$$

Pour le terme $\tilde{T}_{1}^{\mathrm{v}}$ on a

$$
\begin{aligned}
\tilde{T}_{1}^{\mathrm{v}} & =\sum_{q \geq-1} 2^{-q} \sum_{\left|q-q^{\prime}\right| \leq 4} \sum_{-1 \leq j \leq q^{\prime}} \int \triangle_{q}^{\mathrm{v}}\left(\triangle_{j}^{\mathrm{v}} w^{3} \partial_{3} \triangle_{q^{\prime}}^{\mathrm{v}} u\right) \triangle_{q}^{\mathrm{v}} w d x \\
& \leq \sum_{q \geq-1} 2^{-q} \sum_{\left|q-q^{\prime}\right| \leq 4} \sum_{j} \int\left|\triangle_{q}^{\mathrm{v}}\left(\triangle_{j}^{\mathrm{v}} w^{3} \partial_{3} \triangle_{q^{\prime}}^{\mathrm{v}} u\right) \triangle_{q}^{\mathrm{v}} w\right| d x
\end{aligned}
$$

Définissons alors

$$
\begin{aligned}
& \tilde{T}_{1,0}^{\mathrm{v}}=\sum_{q} 2^{-q} \sum_{\left|q-q^{\prime}\right| \leq 4} \int\left|\triangle_{q}^{\mathrm{v}}\left(S_{0}^{\mathrm{v}} w_{3} \partial_{3} \triangle_{q^{\prime}}^{\mathrm{v}} u\right) \triangle_{q}^{\mathrm{v}} w\right| d x \\
& \tilde{T}_{1, N}^{\mathrm{v}}=\sum_{q} \sum_{\left|q-q^{\prime}\right| \leq 4} 2^{-q} \sum_{0 \leq j \leq N} \int\left|\triangle_{q}^{\mathrm{v}}\left(\triangle_{j}^{\mathrm{v}} w_{3} \partial_{3} \triangle_{q^{\prime}}^{\mathrm{v}} u\right) \triangle_{q}^{\mathrm{v}} w\right| d x \quad \text { et } \\
& \tilde{T}_{1}^{\mathrm{v}, \mathrm{N}}=\sum_{q} \sum_{\left|q-q^{\prime}\right| \leq 4} 2^{-q} \sum_{j \geq N} \int\left|\triangle_{q}^{\mathrm{v}}\left(\triangle_{j}^{\mathrm{v}} w_{3} \partial_{3} \triangle_{q^{\prime}}^{\mathrm{v}} u\right) \triangle_{q}^{\mathrm{v}} w\right| d x .
\end{aligned}
$$

On a donc $\tilde{T}_{1}^{\mathrm{v}} \leq \tilde{T}_{1,0}^{\mathrm{v}}+\tilde{T}_{1, N}^{\mathrm{v}}+\tilde{T}_{1}^{\mathrm{v}, \mathrm{N}}$. Estimons d'abord le terme $\tilde{T}_{1,0}^{\mathrm{v}}$. On a

$$
\begin{aligned}
& \tilde{T}_{1,0}^{\mathrm{v}} \leq \sum_{q} 2^{-q} \sum_{\left|q-q^{\prime}\right| \leq 4}\left\|S_{0}^{\mathrm{v}} w^{3}\right\|_{L_{\mathrm{v}}^{\infty} L_{h}^{2}}\left\|\partial_{3} \triangle_{q^{\prime}}^{\mathrm{v}} u\right\|_{L_{\mathrm{v}}^{2} L_{h}^{4}}\left\|\triangle_{q}^{\mathrm{v}} w\right\|_{L_{\mathrm{v}}^{2} L_{h}^{4}} \\
& \leq \sum_{q} 2^{-q} \sum_{\left|q-q^{\prime}\right| \leq 4} C 2^{q^{\prime}}\|w\|_{H^{0,-1 / 2}}\left\|\triangle_{q^{\prime}}^{\mathrm{v}} u\right\|_{L^{2}}^{1 / 2}\left\|\nabla_{h} \triangle_{q^{\prime}}^{\mathrm{v}} u\right\|_{L^{2}}^{1 / 2}\left\|\triangle_{q}^{\mathrm{v}} w\right\|_{L^{2}}^{1 / 2}\left\|\nabla_{h} \triangle_{q}^{\mathrm{v}} w\right\|_{L^{2}}^{1 / 2} .
\end{aligned}
$$

Compte tenu du fait que $u$ appartient à l'espace $H^{0,1 / 2}$ et $w$ est estimée dans l'espace $H^{0,-1 / 2}$, on obtient

$$
\begin{aligned}
\tilde{T}_{1,0}^{\mathrm{v}} & \leq C\|u\|_{H^{0,1 / 2}}^{1 / 2}\left\|\nabla_{h} u\right\|_{H^{0,1 / 2}}^{1 / 2}\|w\|_{H^{0,-1 / 2}}^{3 / 2}\left\|\nabla_{h} w\right\|_{H^{0,-1 / 2}}^{1 / 2} \\
& \leq C\|u\|_{H^{0,1 / 2}}^{2 / 3}\left\|\nabla_{h} u\right\|_{H^{0,1 / 2}}^{2 / 3}\|w\|_{H^{0,-1 / 2}}^{2}+\frac{\nu_{h}}{50}\left\|\nabla_{h} w\right\|_{H^{0,-1 / 2}}^{2} .
\end{aligned}
$$


Comme $\|u(t)\|_{H^{0,1 / 2}}^{2 / 3}\left\|\nabla_{h} u(t)\right\|_{H^{0,1 / 2}}^{2 / 3} \leq C f(t)$, on obtient

$$
\tilde{T}_{1,0}^{\mathrm{v}} \leq C f(t)\|w\|_{H^{0,-1 / 2}}^{2}+\frac{\nu_{h}}{50}\left\|\nabla_{h} w\right\|_{H^{0,-1 / 2}}^{2} .
$$

Estimons maintenant $\tilde{T}_{1, N}^{\mathrm{v}}$. Pour faire cela, soit $\varepsilon<1 / 2$, un paramètre qui sera choisi à la fin. L'inégalité de Hölder nous donne

$$
\begin{aligned}
\tilde{T}_{1, N}^{\mathrm{v}} & \leq \sum_{q \geq-1} \sum_{\left|q-q^{\prime}\right| \leq 4} 2^{-q} \sum_{0 \leq j \leq N}\left\|\triangle_{q}^{\mathrm{v}}\left(\triangle_{j}^{\mathrm{v}} w^{3} \partial_{3} \triangle_{q^{\prime}}^{\mathrm{v}} u\right)\right\|_{L_{\mathrm{v}}^{2} L_{h}^{\frac{2}{1+\varepsilon}}}\left\|\triangle_{q}^{\mathrm{v}} w\right\|_{L_{\mathrm{v}}^{2} L_{h}^{\frac{2}{1-\varepsilon}}} \\
& \leq \sum_{q \geq-1} \sum_{\left|q-q^{\prime}\right| \leq 4} 2^{-q} \sum_{0 \leq j \leq N}\left\|\triangle_{j}^{\mathrm{v}} w^{3}\right\|_{L_{\mathrm{v}}^{\infty} L_{h}^{2}}\left\|\partial_{3} \triangle_{q^{\prime}}^{\mathrm{v}} u\right\|_{L_{\mathrm{v}}^{2} L_{h}^{\frac{2}{\varepsilon}}}\left\|\triangle_{q}^{\mathrm{v}} w\right\|_{L_{\mathrm{v}}^{2} L_{h}^{\frac{2}{1-\varepsilon}}}
\end{aligned}
$$

L'inégalité de Gagliardo-Nirenberg nous donne

$$
\begin{aligned}
\tilde{T}_{1, N}^{\mathrm{v}} \leq \frac{C}{\sqrt{\varepsilon}} \sum_{q \geq-1} \sum_{\left|q-q^{\prime}\right| \leq 4} 2^{-q} \sum_{0 \leq j \leq N} & \left\|\triangle_{j}^{\mathrm{v}} w^{3}\right\|_{L_{\mathrm{v}}^{\infty} L_{h}^{2}}\left\|\partial_{3} \triangle_{q^{\prime}}^{\mathrm{v}} u\right\|_{L^{2}}^{\varepsilon} \times \\
& \times\left\|\partial_{3} \triangle_{q^{\prime}}^{\mathrm{v}} \nabla_{h} u\right\|_{L^{2}}^{1-\varepsilon}\left\|\triangle_{q}^{\mathrm{v}} w\right\|_{L^{2}}^{1-\varepsilon}\left\|\triangle_{q}^{\mathrm{v}} \nabla_{h} w\right\|_{L^{2}}^{\varepsilon}
\end{aligned}
$$

En utilisant le lemme 3.2 et le fait que $u$ appartient à l'espace $H^{0,1 / 2}$, on a

$$
\begin{aligned}
\left\|\partial_{3} \triangle_{q^{\prime}}^{\mathrm{v}} u\right\|_{L^{2}}^{\varepsilon}\left\|\partial_{3} \triangle_{q^{\prime}}^{\mathrm{v}} \nabla_{h} u\right\|_{L^{2}}^{1-\varepsilon} & \leq C 2^{q^{\prime}}\left\|\triangle_{q^{\prime}}^{\mathrm{v}} u\right\|_{L^{2}}^{\varepsilon}\left\|\triangle_{q^{\prime}}^{\mathrm{v}} \nabla_{h} u\right\|_{L^{2}}^{1-\varepsilon} \\
& \leq C 2^{q^{\prime} / 2} c_{q^{\prime}}\|u\|_{H^{0,1 / 2}}^{\varepsilon}\left\|\nabla_{h} u\right\|_{H^{0,1 / 2}}^{1-\varepsilon}
\end{aligned}
$$

avec $c_{q^{\prime}}$ suite de carré sommable.

D'autre part, comme $w$ est estimée dans $H^{0,-1 / 2}$, on a

$$
\left\|\triangle_{q}^{\mathrm{v}} w\right\|_{L^{2}}^{1-\varepsilon}\left\|\triangle_{q}^{\mathrm{v}} \nabla_{h} w\right\|_{L^{2}}^{\varepsilon} \leq C 2^{q / 2} \tilde{c}_{q}\|w\|_{H^{0,-1 / 2}}^{1-\varepsilon}\left\|\nabla_{h} w\right\|_{H^{0,-1 / 2}}^{\varepsilon},
$$

où $\tilde{c}_{q}$ est une suite avec $\sum_{q} \tilde{c}_{q}^{2}=1$.

Il nous reste à estimer $\sum_{0 \leq j \leq N}\left\|\triangle_{j}^{\mathrm{v}} w^{3}\right\|_{L_{\mathrm{v}}^{\infty} L_{h}^{2}}$. Le lemme de Bernstein (lemme 3.2), nous donne

$$
\sum_{0 \leq j \leq N}\left\|\triangle_{j}^{\mathrm{v}} w^{3}\right\|_{L_{\mathrm{v}}^{\infty} L_{h}^{2}} \leq C \sum_{0 \leq j \leq N} 2^{j / 2}\left\|\triangle_{j}^{\mathrm{v}} w^{3}\right\|_{L^{2}} \leq C \sum_{0 \leq j \leq N} 2^{-j / 2}\left\|\triangle_{j}^{\mathrm{v}} \partial_{3} w^{3}\right\|_{L^{2}} .
$$

La condition de divergence nulle sur $w$ nous donne

$$
\sum_{0 \leq j \leq N}\left\|\triangle_{j}^{\mathrm{v}} w^{3}\right\|_{L_{\mathrm{v}}^{\infty} L_{h}^{2}} \leq C \sum_{0 \leq j \leq N} 2^{-j / 2}\left\|\triangle_{j}^{\mathrm{v}} \operatorname{div}_{h} w^{h}\right\|_{L^{2}} \leq C \sqrt{N}\left\|\nabla_{h} w\right\|_{H^{0,-1 / 2}} .
$$


On obtient ainsi l'estimation

$$
\begin{aligned}
\tilde{T}_{1, N}^{\mathrm{v}} \leq C \sqrt{\frac{N}{\varepsilon}}\left\|\nabla_{h} w\right\|_{H^{0,-1 / 2}} \sum_{q \geq-1} \tilde{c}_{q}\left(\sum_{\left|q-q^{\prime}\right| \leq 4} c_{q^{\prime}}\right)\|u\|_{H^{0,1 / 2}}^{\varepsilon} \times \\
\times\left\|\nabla_{h} u\right\|_{H^{0,1 / 2}}^{1-\varepsilon}\|w\|_{H^{0,-1 / 2}}^{1-\varepsilon}\left\|\nabla_{h} w\right\|_{H^{0,-1 / 2}}^{1+\varepsilon} \\
\leq C \sqrt{\frac{N}{\varepsilon}}\left\|\nabla_{h} w\right\|_{H^{0,-1 / 2}}\|u\|_{H^{0,1 / 2}}^{\varepsilon}\left\|\nabla_{h} u\right\|_{H^{0,1 / 2}}^{1-\varepsilon}\|w\|_{H^{0,-1 / 2}}^{1-\varepsilon}\left\|\nabla_{h} w\right\|_{H^{0,-1 / 2}}^{1+\varepsilon} .
\end{aligned}
$$

En utilisant l'inégalité $a b \leq a^{\frac{2}{1+\varepsilon}}+b^{\frac{2}{1-\varepsilon}}$, on obtient

$$
\tilde{T}_{1, N}^{\mathrm{v}} \leq c\left(\frac{N}{\varepsilon}\right)^{\frac{1}{1-\varepsilon}}\|u\|_{H^{0,1 / 2}}^{\frac{\varepsilon}{1-\varepsilon}}\left\|\nabla_{h} u\right\|_{H^{0,1 / 2}}^{2}\|w\|_{H^{0,-1 / 2}}^{2}+\frac{\nu_{h}}{100}\left\|\nabla_{h} w\right\|_{H^{0,-1 / 2}}^{2} .
$$

En faisant le choix $\varepsilon=\frac{1}{1+\ln N}$, on obtient

$$
\tilde{T}_{1, N}^{\mathrm{v}} \leq C N(1+\ln N) f(t)\|w(t)\|_{H^{0,-1 / 2}}^{2}+\frac{\nu_{h}}{100}\left\|\nabla_{h} w(t)\right\|_{H^{0,-1 / 2}}^{2} .
$$

L'estimation sur $\tilde{T}_{1}^{\mathrm{v}, \mathrm{N}}$ est facile et se fait de manière classique. En effet, on peut écrire

$$
\tilde{T}_{1}^{\mathrm{v}, \mathrm{N}} \leq \sum_{q \geq-1} 2^{-q} \sum_{\left|q-q^{\prime}\right| \leq 4} \sum_{j \geq N}\left\|\triangle_{j}^{\mathrm{v}} w^{3}\right\|_{L_{\mathrm{v}}^{\infty} L_{h}^{2}}\left\|\partial_{3} \triangle_{q^{\prime}}^{\mathrm{v}} u\right\|_{L_{\mathrm{v}}^{2} L_{h}^{4}}\left\|\triangle_{q}^{\mathrm{v}} w\right\|_{L_{\mathrm{v}}^{2} L_{h}^{4}} .
$$

Vu que $\nabla_{h} w$ est dans l'espace $H^{0,1 / 2}$, on a l'estimation suivante

$$
\begin{aligned}
\sum_{j \geq N}\left\|\triangle_{j}^{\mathrm{v}} w^{3}\right\|_{L_{\mathrm{v}}^{\infty} L_{h}^{2}} & \leq C \sum_{j \geq N} 2^{j / 2}\left\|\triangle_{j}^{\mathrm{v}} w^{3}\right\|_{L^{2}} \leq C \sum_{j \geq N} 2^{-j / 2}\left\|\triangle_{j}^{\mathrm{v}} \partial_{3} w^{3}\right\|_{L^{2}} \\
& \leq C \sum_{j \geq N} 2^{-j} 2^{j / 2}\left\|\triangle_{j}^{\mathrm{v}} \nabla_{h} w\right\|_{L^{2}} \leq C 2^{-N}\left\|\nabla_{h} w\right\|_{H^{0,1 / 2}} .
\end{aligned}
$$

On obtient ainsi

$$
\begin{aligned}
\tilde{T}_{1}^{\mathrm{v}, \mathrm{N}} \leq C 2^{-N}\left\|\nabla_{h} w\right\|_{H^{0,1 / 2}} \sum_{q \geq-1} 2^{-q} \sum_{\left|q-q^{\prime}\right| \leq 4} 2^{q^{\prime}}\left\|\triangle_{q^{\prime}}^{\mathrm{v}} u\right\|_{L^{2}}^{1 / 2}\left\|\triangle_{q^{\prime}}^{\mathrm{v}} \nabla_{h} u\right\|_{L^{2}}^{1 / 2} \times \\
\times\left\|\triangle_{q}^{\mathrm{v}} w\right\|_{L^{2}}^{1 / 2}\left\|\nabla_{h} \triangle_{q}^{\mathrm{v}} w\right\|_{L^{2}}^{1 / 2} \\
\leq C 2^{-N}\left\|\nabla_{h} w\right\|_{H^{0,1 / 2}}\|u\|_{H^{0,1 / 2}}^{1 / 2}\left\|\nabla_{h} u\right\|^{1 / 2}\|w\|_{H^{0,-1 / 2}}^{1 / 2}\left\|\nabla_{h} w\right\|_{H^{0,-1 / 2}}^{1 / 2} .
\end{aligned}
$$

En utilisant l'inegalité $a b \leq \frac{3}{4} a^{4 / 3}+\frac{1}{4} b^{4}$, on obtient

$$
\tilde{T}_{1}^{\mathrm{v}, \mathrm{N}} \leq C 2^{-4 N / 3} f(t)\|w(t)\|_{H^{0,-1 / 2}}^{2 / 3}+\frac{\nu_{h}}{100}\left\|\nabla_{h} w(t)\right\|_{H^{0,-1 / 2}}^{2} .
$$


Avec le choix de $N=-\ln \|w(t)\|_{H^{0,-1 / 2}}^{2}$, on obtient finalement

$$
\begin{aligned}
\tilde{T}_{1}^{\mathrm{v}} \leq C f(t)\|w(t)\|_{H^{0,-1 / 2}}^{2}\left(1-\ln \|w(t)\|_{H^{0,-1 / 2}}^{2}\right) & \ln \left(1-\ln \|w(t)\|_{H^{0,-1 / 2}}^{2}\right) \\
& +\frac{\nu_{h}}{50}\left\|\nabla_{h} w(t)\right\|_{H^{0,-1 / 2}}^{2} .
\end{aligned}
$$

Pour estimer $\tilde{T}_{2}^{\mathrm{v}}$, on commence par écrire $\tilde{T}_{2}^{\mathrm{v}} \leq \tilde{T}_{2, N}^{\mathrm{v}}+\tilde{T}_{2}^{\mathrm{v}, \mathrm{N}}$, où on a noté

$$
\tilde{T}_{2, N}^{\mathrm{v}}=\sum_{q \leq N} 2^{-q} \sum_{\left|q-q^{\prime}\right| \leq 4} \int\left|\triangle_{q}^{\mathrm{v}}\left(S_{q^{\prime}-1}^{\mathrm{v}} \partial_{3} u \triangle_{q^{\prime}}^{\mathrm{v}} w^{3}\right) \triangle_{q}^{\mathrm{v}} w\right| d x
$$

respectivement

$$
\tilde{T}_{2}^{\mathrm{v}, \mathrm{N}}=\sum_{q \geq N} 2^{-q} \sum_{\left|q-q^{\prime}\right| \leq 4} \int\left|\triangle_{q}^{\mathrm{v}}\left(S_{q^{\prime}-1}^{\mathrm{v}} \partial_{3} u \triangle_{q^{\prime}}^{\mathrm{v}} w^{3}\right) \triangle_{q}^{\mathrm{v}} w\right| d x .
$$

Le terme $\tilde{T}_{2, N}^{\mathrm{v}}$ est majoré comme suit

$$
\begin{aligned}
\tilde{T}_{2, N}^{\mathrm{v}} & \leq \sum_{q \leq N} 2^{-q} \sum_{\left|q-q^{\prime}\right| \leq 4}\left\|\triangle_{q}^{\mathrm{v}}\left(S_{q^{\prime}-1}^{\mathrm{v}} \partial_{3} u \cdot \triangle_{q^{\prime}}^{\mathrm{v}} w^{3}\right)\right\|_{L_{\mathrm{v}}^{2} L_{h}^{\frac{2}{1+\varepsilon}}}\left\|\triangle_{q}^{\mathrm{v}} w\right\|_{L_{\mathrm{v}}^{2} L_{h}^{\frac{2}{1-\varepsilon}}} \\
& \leq \sum_{q \leq N} 2^{-q} \sum_{\left|q-q^{\prime}\right| \leq 4}\left\|S_{q^{\prime}-1}^{\mathrm{v}} \partial_{3} u\right\|_{L_{\mathrm{v}}^{\infty} L_{h}^{\frac{2}{\varepsilon}}}\left\|\triangle_{q^{\prime}}^{\mathrm{v}} w^{3}\right\|_{L^{2}}\left\|\triangle_{q}^{\mathrm{v}} w\right\|_{L_{\mathrm{v}}^{2} L_{h}^{\frac{2}{1-\varepsilon}}} .
\end{aligned}
$$

En tenant compte de l'inégalité de Gagliardo-Nirenberg, on obtient

$$
\begin{array}{r}
\tilde{T}_{2, N}^{\mathrm{v}} \leq \frac{C}{\sqrt{\varepsilon}} \sum_{-1 \leq q \leq N} 2^{-q} \sum_{\left|q-q^{\prime}\right| \leq 4}\left\|S_{q^{\prime}-1}^{\mathrm{v}} \partial_{3} u\right\|_{L_{\mathrm{v}}^{\infty} L_{h}^{2}}^{\varepsilon}\left\|S_{q^{\prime}-1}^{\mathrm{v}} \partial_{3} \nabla_{h} u\right\|_{L_{\mathrm{v}}^{\infty} L_{h}^{2}}^{1-\varepsilon} \times \\
\times\left\|\triangle_{q^{\prime}}^{\mathrm{v}} w^{3}\right\|_{L^{2}}\left\|\triangle_{q}^{\mathrm{v}} w\right\|_{L^{2}}^{1-\varepsilon}\left\|\triangle_{q}^{\mathrm{v}} \nabla_{h} w\right\|_{L^{2}}^{\varepsilon} .
\end{array}
$$

Comme on a

$$
\begin{aligned}
\left\|S_{q^{\prime}-1}^{\mathrm{v}} \partial_{3} u\right\|_{L^{\infty} L_{h}^{2}}^{\varepsilon}\left\|S_{q^{\prime}-1}^{\mathrm{v}} \partial_{3} \nabla_{h} u\right\|_{L_{\mathrm{v}}^{\infty} L_{h}^{2}}^{1-\varepsilon} & \leq C 2^{q^{\prime}}\left\|S_{q^{\prime}-1}^{\mathrm{v}} u\right\|_{L_{\mathrm{v}}^{\infty} L_{h}^{2}}^{\varepsilon}\left\|S_{q^{\prime}-1}^{\mathrm{v}} \nabla_{h} u\right\|_{L_{\mathrm{v}}^{\infty} L_{h}^{2}}^{1-\varepsilon} \\
& \leq C 2^{q^{\prime}} \sqrt{q^{\prime}}\|u\|_{H^{0,1 / 2}}^{\varepsilon}\left\|\nabla_{h} u\right\|_{H^{0,1 / 2}}^{1-\varepsilon}
\end{aligned}
$$

et

$$
\begin{aligned}
\left\|\triangle_{q^{\prime}}^{\mathrm{v}} w^{3}\right\|_{L^{2}} \leq C 2^{-q^{\prime}}\left\|\triangle_{q^{\prime}}^{\mathrm{v}} \partial_{3} w^{3}\right\|_{L^{2}} & \leq C 2^{-q^{\prime}}\left\|\triangle_{q^{\prime}}^{\mathrm{v}} \nabla_{h} w\right\|_{L^{2}} \\
& \leq C 2^{-q^{\prime} / 2} c_{q^{\prime}}\left\|\nabla_{h} w\right\|_{H^{0,-1 / 2}}
\end{aligned}
$$

où $c_{q^{\prime}} \in l^{2}(\mathbb{N})$, on obtient

$$
\begin{aligned}
\tilde{T}_{2, N}^{\mathrm{v}} \leq \frac{C}{\sqrt{\varepsilon}} \sum_{q \leq N} \sum_{\left|q-q^{\prime}\right| \leq 4} 2^{-q^{\prime} / 2} & \sqrt{q^{\prime}} c_{q^{\prime}}\|u\|_{H^{0,1 / 2}}^{\varepsilon}\left\|\nabla_{h} u\right\|_{H^{0,1 / 2}}^{1-\varepsilon} \times \\
& \times\left\|\nabla_{h} w\right\|_{H^{0,-1 / 2}}\left\|\triangle_{q}^{\mathrm{v}} w\right\|_{L^{2}}^{1-\varepsilon}\left\|\triangle_{q}^{\mathrm{v}} \nabla_{h} w\right\|_{L^{2}}^{\varepsilon} .
\end{aligned}
$$


D'autre part, on a

$$
\left\|\triangle_{q}^{\mathrm{v}} w\right\|_{L^{2}}^{1-\varepsilon}\left\|\triangle_{q}^{\mathrm{v}} \nabla_{h} w\right\|_{L^{2}}^{\varepsilon} \leq C 2^{q / 2} \tilde{c}_{q}\|w\|_{H^{0,-1 / 2}}^{1-\varepsilon}\left\|\nabla_{h} w\right\|_{H^{0,-1 / 2}}^{\varepsilon}
$$

avec $\tilde{c}_{q} \in l^{2}(\mathbb{N})$. On a obtenu ainsi l'inégalité

$$
\begin{aligned}
\tilde{T}_{2, N}^{\mathrm{v}} & \leq C \sqrt{\frac{N}{\varepsilon}}\|u\|_{H^{0,1 / 2}}^{\varepsilon}\left\|\nabla_{h} u\right\|_{H^{0,1 / 2}}^{1-\varepsilon}\|w\|_{H^{0,-1 / 2}}^{1-\varepsilon}\left\|\nabla_{h} w\right\|_{H^{0,-1 / 2}}^{\varepsilon} \\
& \leq C\left(\frac{N}{\varepsilon}\right)^{\frac{1}{1-\varepsilon}}\|u\|_{H^{0,1 / 2}}^{\frac{\varepsilon}{1-\varepsilon}}\left\|\nabla_{h} u\right\|_{H^{0,1 / 2}}^{2}\|w\|_{H^{0,-1 / 2}}^{2}+\frac{\nu_{h}}{100}\left\|\nabla_{h} w\right\|_{H^{0,-1 / 2}}^{2} .
\end{aligned}
$$

Avec le choix de $\varepsilon=\frac{1}{1+\ln N}$, on obtient

$$
\tilde{T}_{2}^{\mathrm{v}} \leq C N(1+\ln N) f(t)\|w\|_{H^{0,-1 / 2}}^{2}+\frac{\nu_{h}}{100}\left\|\nabla_{h} w\right\|_{H^{0,-1 / 2}}^{2} .
$$

Le terme $\tilde{T}_{2}^{\mathrm{v}, \mathrm{N}}$ se majore de façon classique. On obtient facilement

$$
\begin{aligned}
\tilde{T}_{2}^{\mathrm{v}, \mathrm{N}} & \leq 2^{-N / 2} f(t)^{3 / 4}\|w\|_{H^{0,-1 / 2}}^{1 / 2}\left\|\nabla_{h} w\right\|_{H^{0,-1 / 2}}^{1 / 2} \\
& \leq 2^{-2 N / 3} f(t)\|w\|_{H^{0,-1 / 2}}^{2 / 3}+\frac{\nu_{h}}{100}\left\|\nabla_{h} w\right\|_{H^{0,-1 / 2}}^{2} .
\end{aligned}
$$

Avec le choix de $N=-\ln \|w\|_{H^{0,-1 / 2}}^{2}$, on obtient finalement

(5.20) $\left.\tilde{T}_{2}^{\mathrm{v}} \leq C f(t)\|w(t)\|_{H^{0,-1 / 2}}^{2}\left(1-\ln \|w(t)\|_{H^{0,-1 / 2}}^{2}\right)\right) \ln \left(1-\ln \|w(t)\|_{H^{0,-1 / 2}}^{2}\right)$.

L'estimation sur $\tilde{R}^{\mathrm{v}}$ se fait de la même manière que pour le terme $R^{h}$, après s'être ramené à un terme horizontal. En effet, pour se ramener à un terme horizontal, on utilise toujours

$$
\left\|\partial_{3} \triangle_{q}^{\mathrm{v}} u\right\|_{L_{\mathrm{v}}^{p} L_{h}^{r}} \leq C 2^{q}\left\|\triangle_{q}^{\mathrm{v}} u\right\|_{L_{\mathrm{v}}^{p} L_{h}^{r}}
$$

et pour des champs de divergence nulle

$$
\left\|\triangle_{q}^{\mathrm{v}} w^{3}\right\|_{L_{\mathrm{v}}^{p} L_{h}^{r}} \leq C 2^{-q}\left\|\triangle_{q}^{\mathrm{v}} \nabla_{h} w\right\|_{L_{\mathrm{v}}^{p} L_{h}^{r}} \quad \text { pour tout } \quad q \geq 0 .
$$

Maintenant, la majoration sur $\tilde{R}^{\mathrm{v}}$ se fait comme pour le terme $R^{h}$. On obtient ainsi

$$
\tilde{R}^{\mathrm{v}} \leq C f(t)\|w\|_{H^{0,-1 / 2}}^{2}\left(1-\ln \|w\|_{H^{0,-1 / 2}}^{2}\right) \ln \left(1-\ln \|w\|_{H^{0,-1 / 2}}^{2}\right) .
$$

En sommant toutes les estimations (5.10)-(5.21), on obtient avec la notation $\varphi(t)=\|w(t)\|_{H^{0,-1 / 2}}^{2}$,

$$
\varphi^{\prime}(t) \leq C f(t) \varphi(t)(1-\ln \varphi(t)) \ln (1-\ln \varphi(t))
$$

avec $f \in L_{\text {loc }}^{1}$. 


\section{Relations entre les espaces}

Rappelons les espaces Besov isotropes $B_{p, q}^{s}\left(\mathbb{R}^{d}\right)$ qui sont définis ainsi

\section{Définition 6.1}

$$
B_{p, q}^{s}\left(\mathbb{R}^{d}\right)=\left\{u \in \mathcal{S}^{\prime}\left(\mathbb{R}^{d}\right) \mid\left(\sum_{k \in \mathbb{Z}} 2^{k q s}\left\|\triangle_{k} u\right\|_{L^{p}}^{q}\right)^{\frac{1}{q}}<\infty\right\}
$$

où nous avons défini les opérateurs $\triangle_{k}$ par $\triangle_{k} u=\mathcal{F}^{-1}\left(\varphi\left(\frac{|\xi|}{2^{k}}\right) \mathcal{F} u(\xi)\right)$.

Nous rappelons aussi, que les espaces anisotropes de type Besov ont été introduits dans [11]. Il s'agit des espaces $H B^{s, s^{\prime}}$ qui sont introduits par la norme :

$$
\|u\|_{H B^{s, s^{\prime}}}:=\left\|2^{k s+q s^{\prime}}\right\| \triangle_{k}^{h} \triangle_{q}^{\mathrm{v}} u\left\|_{L^{2}}\right\|_{\ell_{k}^{2}\left(\ell_{q}^{1}\right)}<\infty
$$

où les opérateurs de troncatures en fréquences horizontales sont définis comme suit :

$$
\triangle_{k}^{h} a=\mathcal{F}^{-1}\left(\varphi\left(2^{-k}\left|\xi_{h}\right|\right) \mathcal{F} a(\xi)\right) .
$$

Il faut remarquer que l'espace $\mathcal{B}^{0,1 / 2}$ est un sous-espace de $H B^{0,1 / 2}$, avec l'inclusion continue.

Nous allons démontrer d'abord qu'on a l'inclusion $\mathcal{B}^{0,1 / 2} \hookrightarrow C^{-1}$. Pour cela, il suffit de démontrer :

$$
\left\|S_{q} u\right\|_{L^{\infty}} \leq 2^{q}\|u\|_{\mathcal{B}^{0,1 / 2}} .
$$

On considère une fonction réguliere $\tilde{\chi}$ qui vaut 1 sur la boule $B(0,4 / 3)$ et qui a le support inclus dans une boule $\tilde{B}$. On peut écrire alors $S_{q} u=\tilde{S}_{q}^{h} \tilde{S}_{q}^{\text {v }} S_{q} u$, où $\tilde{S}_{q}^{h}$ et $\tilde{S}_{q}^{\mathrm{v}}$ sont les opérateurs de localisation en fréquences horizontale respectivement en fréquences verticales associés à la fonction $\tilde{\chi}$. En utilisant le lemme 3.2, nous avons :

$$
\begin{aligned}
\left\|S_{q} u\right\|_{L^{\infty}} & \leq C 2^{q}\left\|\tilde{S}_{q}^{\mathrm{v}} u\right\|_{L_{h}^{2}\left(L_{\mathrm{v}}^{\infty}\right)} \leq C 2^{q} \sum_{j \leq q}\left\|\tilde{\triangle}_{j}^{\mathrm{v}} u\right\|_{L_{h}^{2}\left(L_{\mathrm{v}}^{\infty}\right)} \\
& \leq C 2^{q} \sum_{j \leq q} 2^{j / 2}\left\|\tilde{\triangle}_{j}^{\mathrm{v}} u\right\|_{L^{2}} \leq C 2^{q}\|u\|_{\mathcal{B}^{0,1 / 2}} .
\end{aligned}
$$

Pour démontrer la non-inclusion de $\mathcal{B}^{0,1 / 2}$ dans les espaces de Besov isotropes $B_{p, \infty}^{3 / p-1}$, il suffit de démontrer ceci pour $p>3$ (on a $B_{r, \infty}^{3 / r-1} \subset B_{p, \infty}^{3 / p-1}$ pour $r \leq p$ ). Remarquons que pour $p>3$, la norme dans $B_{p, \infty}^{3 / p-1}$ est donnée par

$$
\sup _{q \in \mathbb{Z}}\left(2^{q(-1+3 / p)}\left\|S_{q} u\right\|_{L^{p}}\right)
$$


Supposons qu'on a l'inclusion : $\mathcal{B}^{0,1 / 2} \subset B_{p, \infty}^{3 / p-1}$. Alors, pour toutes les fonctions $u$, on a

$$
2^{(-1+3 / p) q}\left\|S_{q} u\right\|_{L^{p}} \leq\|u\|_{\mathcal{B}^{0,1 / 2}} .
$$

Soit alors $u=f \otimes g$ avec $f \in L^{2}\left(\mathbb{R}_{x_{1}} \times \mathbb{R}_{x_{2}}\right)$ et $g \in \mathcal{B}_{2,1}^{1 / 2}$. Nous avons évidemment $S_{q} u=S_{q}^{h} f \otimes S_{q}^{\mathrm{v}} g$. Alors,

$$
2^{q(-1+3 / p)}\left\|S_{q}^{h} f\right\|_{L^{p}}\left\|S_{q}^{\mathrm{v}} g\right\|_{L^{p}} \leq\|f\|_{L^{2}}\|g\|_{B_{2,1}^{1 / 2}}
$$

Nous choisissons $f_{q}\left(x_{h}\right)=h\left(2^{q} x_{h}\right)$ où $h$ est fonction à décroissance rapide telle que le support de $\mathcal{F} h$ soit inclus dans une petite boule $B$ et $g$ est fonction avec le support de la transformation de Fourier de $g$ inclus dans un compact. On a alors $S_{q}^{h} f_{q}=f_{q}$ et $S_{q}^{\mathrm{v}} g=g$ pour $q$ suffisamment grand. Nous obtenons ainsi que

$$
2^{q(-1+3 / p)}\left\|f_{q}\right\|_{L^{p}}\|g\|_{L^{p}} \leq\left\|f_{q}\right\|_{L^{2}}\|g\|_{B_{2,1}^{1 / 2}} .
$$

Comme

$$
\left\|f_{q}\right\|_{L^{2}\left(\mathbb{R}_{h}^{2}\right)}^{2}=\int\left|h\left(2^{q} x_{h}\right)\right|^{2} d x_{h}=2^{-2 q}\|h\|_{L^{2}\left(\mathbb{R}_{h}^{2}\right)}^{2}
$$

et

$$
\left\|f_{q}\right\|_{L^{p}}=2^{-\frac{2 q}{p}}\|h\|_{L^{p}}
$$

nous obtenons

$$
2^{q(-1+3 / p)} 2^{-2 q / p}\|h\|_{L^{p}\left(\mathbb{R}_{h}^{2}\right)}\|g\|_{L^{p}\left(\mathbb{R}_{\mathrm{v}}\right)} \leq 2^{-q}\|h\|_{L^{2}\left(\mathbb{R}_{h}^{2}\right)}\|g\|_{B_{2,1}^{1 / 2}\left(\mathbb{R}_{\mathrm{v}}\right)} .
$$

En prenant $q$ suffisamment grand, nous avons une contradiction.

Pour démontrer que $H^{0,1 / 2}$ n'est pas inclus dans $C^{-1}$ nous renvoyons à [11]

Lemme 6.1 L'espace $\mathcal{B}^{0,1 / 2}$ est un sous-espace de $\nabla$ BMO.

Je voudrais remercier M. Pierre Gilles Lemarié-Rieusset qui m'a communiqué ce résultat ainsi que la démonstration suivante.

Remarquons d'abord que l'espace de Besov $\mathcal{B}^{0,1 / 2}$ est inclus dans $L_{l o c}^{1}\left(\mathbb{R}^{3}\right)$ parce qu'il est inclus dans $L_{\mathrm{v}}^{\infty} L_{h}^{2}$. En outre, il est contenu dans l'espace de Morrey-Campanato $M^{1,3}$ où l'espace homogène de Morrey-Campanato est défini par la norme

$$
\|f\|_{M^{p, q}}=\sup _{x_{0} \in \mathbb{R}^{n}} \sup _{0<R<1} R^{n(1 / q-1 / p)}\|f\|_{L^{p}\left(B\left(x_{0}, R\right)\right)}<+\infty .
$$

D'autre part, l'espace de Morrey-Campanato $M^{1,3}$ est inclus dans l'espace $\nabla B M O$. Pour la démonstration de cette inclusion nous renvoyons à [15] (théorème 18.2, page 180). 


\section{Références}

[1] Bony, J.-M. : Calcul symbolique et propagation des singularités pour les équations aux dérivées partielles non linéaires. Ann. Sci. École Norm. Sup. (4) 14 (1981), no. 2, 209-246.

[2] Cannone, M. : Ondelettes, paraproduits et Navier-Stokes, Nouveaux essais. Diderot Éditeur, Paris, 1995.

[3] Chemin, J.-Y. : Fluides Parfaits Incompressibles. Astérisque 230, 1995.

[4] Chemin, J.-Y. : Théorèmes d'unicité pour le système de Navier-Stokes tridimensionel. J. Anal. Math. 77 (1999), 27-50.

[5] Chemin, J.-Y. And Lerner, N. : Flot de champs de vecteurs non Lipschitziens et équations de Navier-Stokes. J. Differential Equations 121 (1992), no. $2,314-328$.

[6] Chemin, J.-Y., Desjardins, B., Gallagher, I. and Grenier, E. : Fluids with anisotropic viscosity. M2AN Math. Model Numer. Anal. 34 (2000), no. 2, 315-335.

[7] Chemin, J.-Y. And Xu, C.-J. : Inclusions de Sobolev en calcul de WeylHormander et champs de vecteurs sous-elliptiques. Ann. Sci. École Norm. Sup. (4) 30 (1997), no. 6, 719-751.

[8] Fleet, T. M. : Differential analysis. Cambridge University Press, 1980.

[9] Fujita, H. And Kato, T. : On the Navier-Stokes initial value problem I. Arch. Rational Mech. Anal. 16 (1964), 269-315.

[10] Grenier, E. And Masmoudi, N. : Ekman layers of rotating fluid, the case of well prepared initial data. Comm. Partial Differential Equations 22 (1997), no. 5-6, 953-975.

[11] Iftimie, D. : Resolution of the Navier-Stokes equations in anisotropic spaces. Rev. Mat. Iberoamericana 15 (1999), no. 1, 1-36.

[12] Iftimie, D. : The 3D Navier-Stokes equations seen as a perturbation of the 2D Navier-Stokes equations. Bull. Soc. Math. France 127 (1999), no. $4,473-517$.

[13] Iftimie, D. : A Uniqueness result for the Navier-Stokes Equations with vanishing vertical viscosity. SIAM J. Math. Analysis 33 (2002), no. 6, 1483 1493.

[14] Koch, H. and Tataru, D. : Well-posedness for the Navier-Stokes equations. Adv. Math. 157 (2001), no. 1, 22-35.

[15] Lemarié-Rieusset, P.-G. : Recent developments in the Navier-Stokes problem. Chapman \& Hall/CRC, Boca Raton, FL., 2002.

[16] Leray, J. : Sur le mouvement d'un liquide visqueux remplissant l'espace. Acta Math. 63 (1934), 193-248.

[17] Pedlosky, J. : Geophysical Fluid Dynamics. Springer Verlag, New York, 1987. 
[18] Planchon, F. : Global strong solutions in Sobolev or Lebesgue spaces to the incompressible Navier-Stokes equations in $\mathbb{R}^{3}$. Ann. Inst. H. Poincaré Anal. Non Linéaire 13 (1996), no. 3, 319-336.

[19] Planchon, F. : Asymptotic behavior of global solutions to the NavierStokes equations in $\mathbb{R}^{3}$. Rev. Mat. Iberoamericana 14 (1998), no. 1, 71-93.

[20] Vishik, M. : Incompressible flows of an ideal fluid with vorticity in borderline spaces of Besov type. Ann. Sci. École Norm. Sup. (4) 32 (1999), no. 6, $769-812$.

Recibido: 20 de febrero de 2003

Marius Paicu

Université Paris Sud

Laboratoire de Mathématiques

Bât. 425

91405 Orsay Cedex, France

Marius.Paicu@math.u-psud.fr 